\title{
Vibrational
}

Phase Contrast CARS
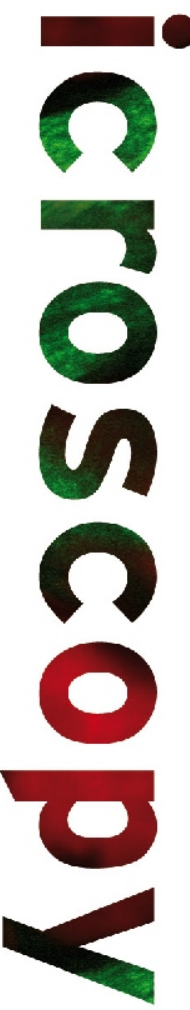


\section{Vibrational Phase Contrast CARS MicRosCOPY}


Samenstelling van de promotiecommissie:

Prof. dr. G. van der Steenhoven Universiteit Twente, Enschede, Nederland Prof. dr. J.L. Herek

Universiteit Twente, Enschede, Nederland

Dr. ir. H.L. Offerhaus

Universiteit Twente, Enschede, Nederland

Dr. C. Otto

Prof. dr. A. Zumbusch

Universiteit Twente, Enschede, Nederland

Prof. dr. J.F. de Boer

Universität Konstanz, Konstanz, Duitsland

Prof. dr. ing. D.H.A. Blank

Vrije Universiteit, Amsterdam, Nederland

Prof. dr. ir. H.J.W. Zandvliet

Universiteit Twente, Enschede, Nederland

Universiteit Twente, Enschede, Nederland

This research was supported by NanoNed, a national nanotechnology program coordinated by the Dutch Ministry of Economic Affairs (project number 6449) and in part by of the "stichting Fundamenteel Onderzoek der Materie" (FOM), which is financially supported by the "Nederlandse Organisatie voor Wetenschappelijk Onderzoek" (NWO).

This work was carried out at:

Optical Sciences group, MESA ${ }^{+}$Institute for Nanotechnology, Faculty of Science and Technology (TNW), University of Twente, The

Netherlands

Cover design: Jeanine van der Hoek

Photo: Maurits Diephuis

ISBN: 978-90-365-3055-2

Author email: mjurna@gmail.com

Copyright (c) 2010 by Martin Jurna

All rights reserved. No part of the material protected by this copyright notice may be reproduced or utilized in any form or by any means, electronic or mechanical, including photocopying, recording or by any information storage and retrieval system, without the prior permission of the author. 


\section{Vibrational Phase Contrast CARS MicRosCOPY}

\section{PROEFSCHRIFT}

ter verkrijging van

de graad van doctor aan de Universiteit Twente, op gezag van de rector magnificus,

prof. dr. H. Brinksma,

volgens besluit van het College voor Promoties

in het openbaar te verdedigen

op vrijdag 2 juli 2010 om 15.00 uur

door

Martin Jurna

geboren op 25 april 1982

te Leeuwarden, Nederland 
Dit proefschrift is goedgekeurd door:

Prof. dr. J.L. Herek (Promotor)

Dr. ir. H.L. Offerhaus (Assistent promotor)

Dr. C. Otto (Assistent promotor) 
aan mijn lieve vrouw Rina 



\section{Contents}

1 Introduction 1

1.1 Light microscopy; the search for chemical specificity . . . 2

1.2 Coherent anti-Stokes Raman scattering . . . . . . . . . . 3

1.3 The vibrational phase . . . . . . . . . . . 6

1.4 Thesis overview . . . . . . . . . . . . . 7

2 The CARS process 9

2.1 Coherent anti-Stokes Raman scattering . . . . . . . . . 10

2.1.1 Nonlinear optics . . . . . . . . . . . . . . 10

2.1.2 The third-order nonlinear susceptibility . . . . . . 10

2.1.3 CARS and Raman . . . . . . . . . . . . . . . . 14

2.2 The non-resonant background . . . . . . . . . . . . 15

2.3 Experimental setup . . . . . . . . . . . . . . . 21

2.3.1 The choice of laser source . . . . . . . . . . 21

2.3 .2 Microscopy setup . . . . . . . . . . . . . 23

3 Heterodyne CARS 27

3.1 Introduction . . . . . . . . . . . . . . . . 28

3.2 Heterodyne detection . . . . . . . . . . . . . . 28

3.2.1 Interferometric detection . . . . . . . . . . . . 28

3.2.2 Cascaded phase preserving chain . . . . . . . . 29

3.3 Shot noise limited detection . . . . . . . . . . . . . 31

3.3 .1 Detectors . . . . . . . . . . . . . 31

3.3.2 Relative intensity noise (RIN) . . . . . . . . . . 38

3.4 Detection improvement . . . . . . . . . . . . . 42

3.5 Phase detection . . . . . . . . . . . . . . . . . 44

3.6 Background rejection . . . . . . . . . . . . 47 
3.7 HIP-CARS . . . . . . . . . . . . . . . . 47

4 Vibrational Phase Contrast CARS 53

4.1 Introduction . . . . . . . . . . . . . . 54

4.2 Vibrational Phase Contrast CARS . . . . . . . . . . 54

4.2.1 Detection of the local excitation phase . . . . . . 54

4.2 .2 Detection setup . . . . . . . . . . . . 56

4.2.3 Imaging of HeLa cells . . . . . . . . . . . . . 58

4.2.4 VPC-CARS in the fingerprint region . . . . . . . 61

4.3 Third order cascaded phase-preserving chain . . . . . . . 63

4.3.1 Single wavelength CARS detection . . . . . . . 63

4.3.2 The cascaded phase-preserving chain . . . . . . . 63

4.3.3 Differences in detection . . . . . . . . . . . 66

4.3.4 Conclusion . . . . . . . . . . . . . 67

4.4 Detection in the complex plane . . . . . . . . . . . 69

4.4.1 The complex plane . . . . . . . . . . . . 69

4.4.2 Monitoring dissolution in the complex plane . . . . 71

4.4.3 Monitoring mixing in the complex plane . . . . . . 73

4.4.4 Multi component analysis . . . . . . . . . . . 74

4.5 Conclusion and Outlook . . . . . . . . . . . 75

4.5.1 Conclusion . . . . . . . . . . . . 75

4.5.2 Outlook ............... 76

5 Applications of CARS microscopy $\quad \mathbf{7 7}$

5.1 CARS as part of a nonlinear microscope system . . . . . 78

5.2 Chemical imaging and monitoring of pharmaceutical tablets 83

5.2 .1 Introduction . . . . . . . . . . . . 83

5.2 .2 Conventional techniques . . . . . . . . . . 85

5.2 .3 Experimental system . . . . . . . . . . . . 85

5.2 .4 Dissolution experiments . . . . . . . . . . . . 88

5.2.5 Conclusion and outlook . . . . . . . . . . 93

5.3 How cells grow bone . . . . . . . . . . . . 96

5.3 .1 Introduction . . . . . . . . . . . . 96

5.3 .2 Previous work . . . . . . . . . . . . 97

5.3 .3 Experimental setup . . . . . . . . . . . . 98

5.3.4 Hypothetical model of how cells grow bone . . . . 100

5.3 .5 Conclusion . . . . . . . . . . . . . . 108

$\begin{array}{lll}6 & \text { Microscale art } & 111\end{array}$ 
$\begin{array}{ll}\text { A Calculations } & \mathbf{1 1 7}\end{array}$

A.1 Noise equivalent Power (NEP) _ . . . . . . . . . 118

A.2 Quantum efficiency $(\mathrm{QE}) \ldots \ldots . \ldots 119$

Bibliography

Summary

Samenvatting

133

Dankwoord

List of publications

141 


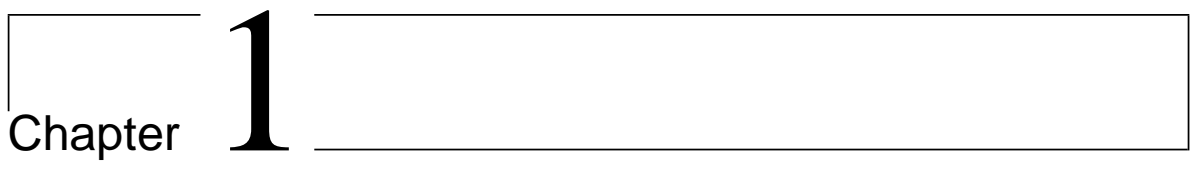

\section{Introduction}

From the moment we open our eyes, our brain analyzes the signals it receives. Visualization of objects, people, and materials is based on recognition of size, color, shape, etc. There are many things, however that we can not see, because they are too small or colorless. To see these things we need a detection method that complements the human eye.

Specificity, selectivity and sensitivity are the three key words in the detection of compounds throughout this thesis. In figure 1.1 these three characteristic words are explained by images. Specificity is the ability to distinguish multiple compounds in the sample, as shown in the figure by four different colors representing four different compounds. Selectivity is the ability to detect one of these compounds, ignoring all others, as shown in the figure by only selecting the compound represented by the color red. Sensitivity is the minimum amount of compound needed to identify the compound. In this thesis a technique is developed that improves specificity, selectivity and sensitivity in microscopy.

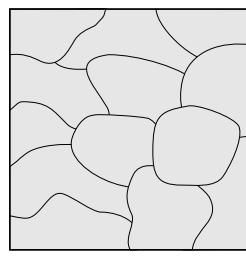

Indistinguishable

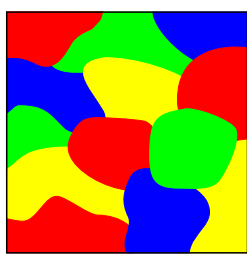

Specificity

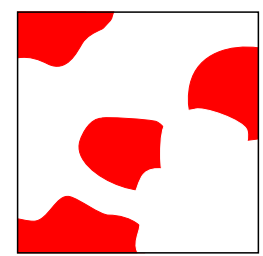

Selectivity

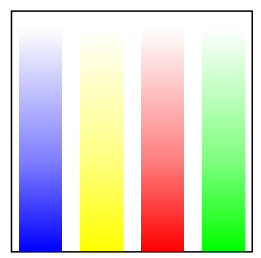

Sensitivity

Figure 1.1: Specificity, selectivity and sensitivity. 


\subsection{Light microscopy; the search for chemical specificity}

A long history of Dutch microscopy could be said to start in 1590 with the discovery, by the lens grinders Hans and Sacharias Jansen, of the magnification obtained by placing two lenses in a tube. It was not until 1675 that the first "real" microscopes were created by Antonie van Leeuwenhoek. He constructed microscopes with up to 500 times magnification and was the first to describe the microbiology of single cells and bacteria. The contrast in the samples was obtained by the differences in the absorption of the transmitted light. In 1933, Frits Zernike improved the contrast in samples by phase-contrast microscopy [1]. This phasecontrast microscopy allowed for the study of colorless and transparent biological materials. The contrast was created by the local variations of the refractive index in the sample.

The specificity in microscopy improved dramatically in the 1940s, when Coons and Kaplan introduced a technique to label antibodies with fluorescent molecules to study antibody-antigen interactions [2]. In 1994, Chalfie and colleagues succeeded in isolating a naturally fluorescent protein in living organisms, known as the green fluorescent protein (GFP) [3]. Whereas most fluorescent labels are highly phototoxic, fluorescent proteins such as GFP are usually much less harmful when illuminated in living cells [4]. This success made fluorescence microscopy into a very important tool in biology studies.

Even though fluorescence microscopy offers high chemical specificity by labeling the specific molecules of interest, there is still a need for other contrast methods. Even though there is a large diversity of fluorescent labels and techniques to label anti bodies, some molecules such as water are difficult to label. Fluorescent labels also limit acquisition time due to photo-bleaching and their presence in the sample can cause perturbation of cell functions or change dissolution behavior from tablets.

Sir C.V. Raman discovered in 1928 that when monochromatic light is incident on molecules, the scattered light contains different colors [5] and a small portion is shifted in frequency. The red-shifted frequencies are called the Stokes scattering, and the blue-shifted frequencies are called the anti-Stokes scattering. The frequency shifts correspond to specific molecular motions that are unique for every type of molecule. The spectrum of these individual and group specific bonds of a molecule 


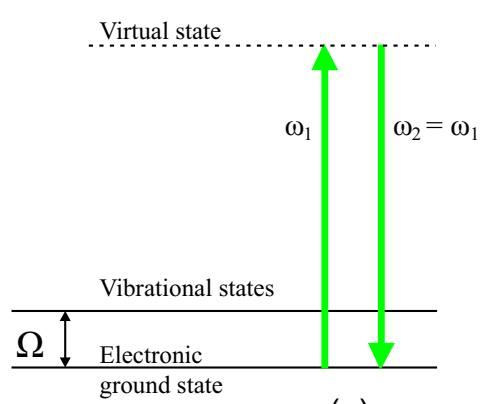

(a)

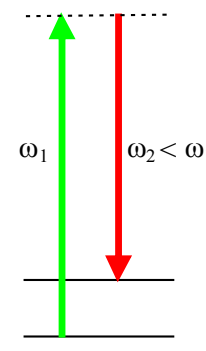

(b)

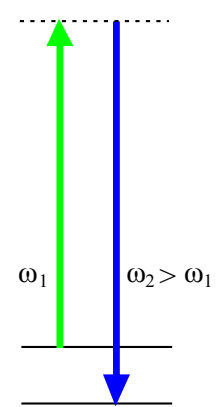

(c)

Figure 1.2: Schematic molecular energy level diagram showing different scattering types $\omega_{2}$ from incident light $\omega_{1}$ on a molecule. (a) Rayleigh scattering, (b) Stokes Raman scattering and (c) anti-Stokes Raman scattering

are sometimes referred to as the 'fingerprint' of a molecule, and can be used to identify the molecule. This discovery therefore offers an alternative method for chemically specific detection.

Figure 1.2 shows the schematic energy level diagrams of the different scattering types. Rayleigh scattering is the most dominant process, with a typical cross section on the order of $10^{-27} \mathrm{~cm}^{2}[6]$. Typical StokesRaman cross-sections are $10^{6}-10^{8}$ times smaller [7]. The intensity of the anti-Stokes Raman scattered light is lower than the Stokes Raman scattered light. This is related to the Boltzmann occupation of the vibrational levels [7]. According to Boltzmann statistics, the population density in the vibrational levels decreases exponentially with the energy of the levels. For this reason Raman spectra are usually recorded at the Stokes side. A major drawback of Stokes spectroscopy is that (auto)fluorescence may obscure the Raman measurements. This drawback is not present with anti-Stokes Raman scattering because the frequency of the anti-Stokes is higher then the frequency of the incident light. A typical Stokes-Raman spectrum of toluene is given in figure 1.3.

\subsection{Coherent anti-Stokes Raman scattering}

After the invention of the laser in 1960 by Maiman [8], the possibility of using stronger electric fields came into reach, opening up possibilities for nonlinear optics and nonlinear vibrational spectroscopy and microscopy. 


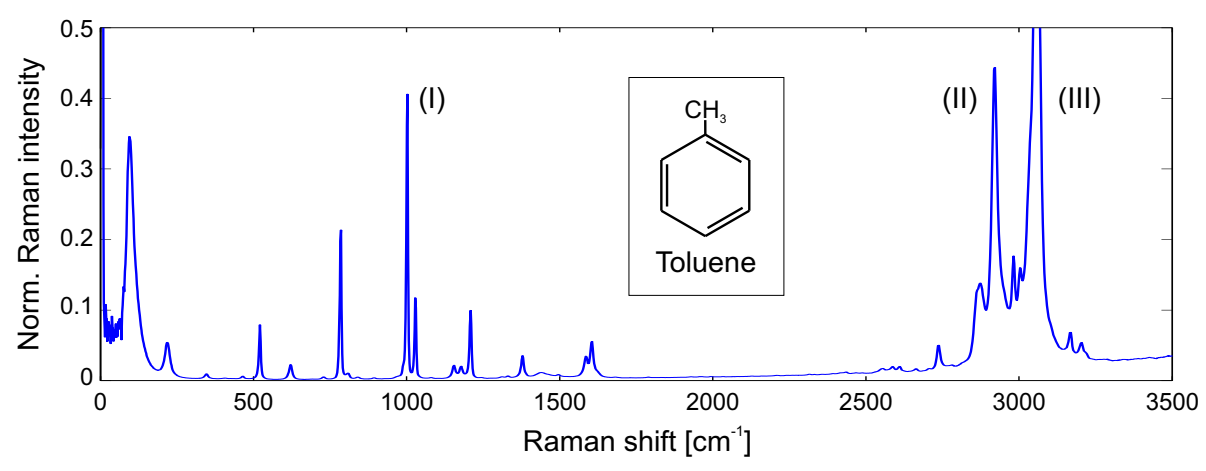

Figure 1.3: Spontaneous Raman spectrum of toluene. In the inset the molecular structure of toluene. (I) Aromatic $\mathrm{C}$-C resonance, (II) $\mathrm{C}-\mathrm{CH}_{3}$ resonance and (III) aromatic $C-H$ resonance.

In 1965 Maker and Terhune of the Scientific Laboratory at the Ford Motor Company demonstrated coherent anti-Stokes Raman scattering (CARS) [9]. CARS is a four-photon process, see figure 1.4(a), where a pump photon of frequency $\omega_{p}$, a Stokes photon of frequency $\omega_{s}$ and probe photon of frequency $\omega_{p r}$ (often taken equal and from the same source as the pump frequency) interact with the sample and generate an anti-Stokes photon of frequency $\omega_{a s}=\omega_{p}-\omega_{s}+\omega_{p r}$. The anti-Stokes or CARS signal is resonantly enhanced when the difference frequency $\left(\omega_{p}-\omega_{s}\right)$ coincides with a molecular vibrational resonance $\Omega$. By tuning the difference frequency $\left(\omega_{p}-\omega_{s}\right)$, the unique vibrational spectrum of a molecule can be obtained. A constant difference frequency corresponding to one of the specific vibrational resonances of the molecule of interest can be used for imaging.

In the CARS process the molecule returns to its initial state, giving an overall conservation of energy and momentum. Because all transitions are driven rather than spontaneous, the process is coherent and has to fulfill phase-matching conditions for efficient generation. The result is that the CARS signal is collimated, which makes detection more efficient compared to spontaneous Raman scattering. Because the CARS signal is blue-shifted compared to the input wavelengths, the detected signal is free of one-photon auto-fluorescence. The major disadvantage of CARS compared to spontaneous Raman scattering is the presence of a non-resonant background signal, shown in the schematic energy level diagram in figure 1.4(b-c). This frequency independent background is created by non-resonant coherent four-wave mixing [10]. In chapter 2 


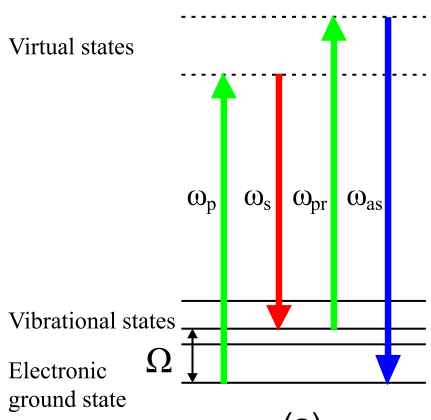

(a)

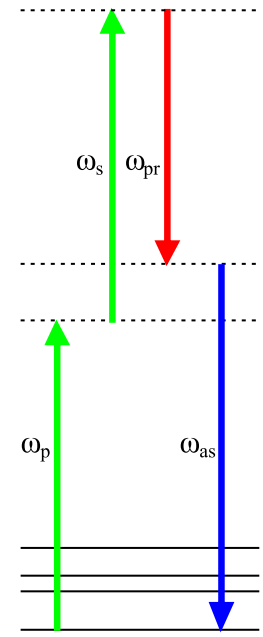

(b)

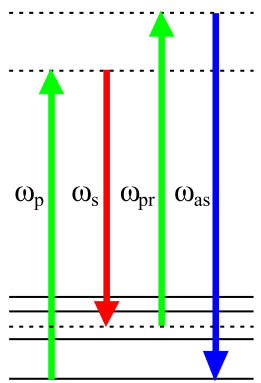

(c)

Figure 1.4: Energy level diagram of CARS. (a) Resonant contribution and (b) non-resonant contribution. (c) Non-resonant contribution provided by other molecules.

the existence of the non-resonant background will be discussed in detail as well as possibilities to reduce it.

CARS microscopy was first demonstrated in 1982 by Duncan [11], but due to the unpractical non-collinear beam geometry, CARS spectroscopy was mostly explored in the $80 \mathrm{~s}$ and $90 \mathrm{~s}$. In the late $90 \mathrm{~s}$ it was shown that the complicated non-collinear geometry could be replaced by a simpler geometry, using a high numerical aperture objective $[12,13]$. This improvement in the simplicity of CARS microscopy has resulted in the rapid development and application of CARS in the last decade.

An example is shown in figure 1.5 that highlights the chemical specificity of CARS microscopy in a sample containing a mixture of $4-\mu \mathrm{m}$ plastic beads. The transmission image, where the contrast is based on the absorption and scattering of the beads, shows the location of the beads, but does not distinguish the two different plastics. By tuning the difference frequency $\omega_{p}-\omega_{s}$, the unique vibrational CARS spectrum of the two plastics is obtained. CARS images are shown for five different locations in the vibrational spectrum. The second image, obtained at a Raman shift of $2895 \mathrm{~cm}^{-1}$, shows all the beads due to the similar vibrational resonance strength of both materials. Images three 


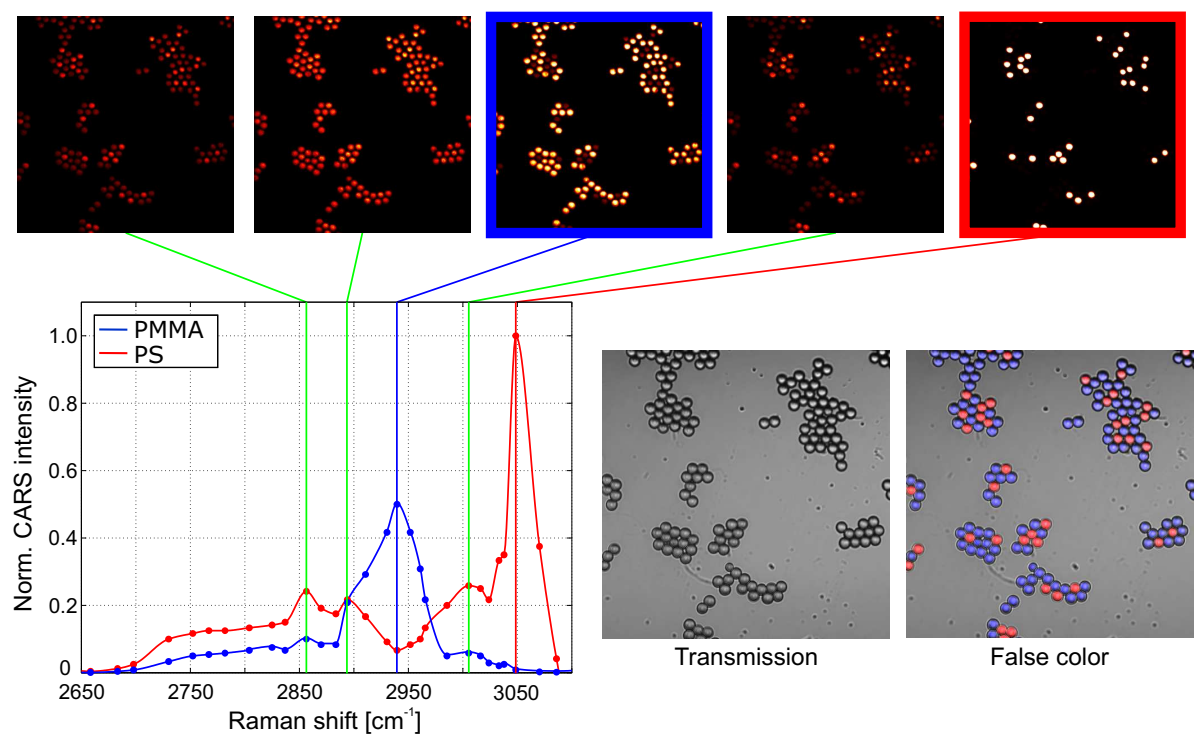

Figure 1.5: An example of chemically selective imaging with CARS microscopy. CARS microscopy images are taken of a mixture of $4 \mu \mathrm{m}$ PMMA (polymethylmethacrylaat) and PS (polystyrene) beads at five different Raman shifts. The images obtained at $2945 \mathrm{~cm}^{-1}$ and $3050 \mathrm{~cm}^{-1}$ show a high selectivity for PMMA and PS, respectively and allow for false color coding of the transmission image.

and five at $2945 \mathrm{~cm}^{-1}$ and $3050 \mathrm{~cm}^{-1}$ respectively, show high selectivity for PMMA (polymethylmethacrylaat) and PS (polystyrene). Coloring the image obtained at $2945 \mathrm{~cm}^{-1}$ blue and the image at $3050 \mathrm{~cm}^{-1}$ red and superimposing them results in a false color image that can be superimposed on the transmission image. This false color image shows which beads are made of each plastic. This example demonstrates the combination of nonlinear vibrational spectroscopy and microscopy to provide a noninvasive method to identify structures of varied chemical composition.

\subsection{The vibrational phase}

When a molecule is driven with an external oscillating (driving) force like a light field, the response is an oscillation of the charge density of the molecule. The maximum displacement from the resting position is called the "amplitude". The phase difference between the applied dri- 

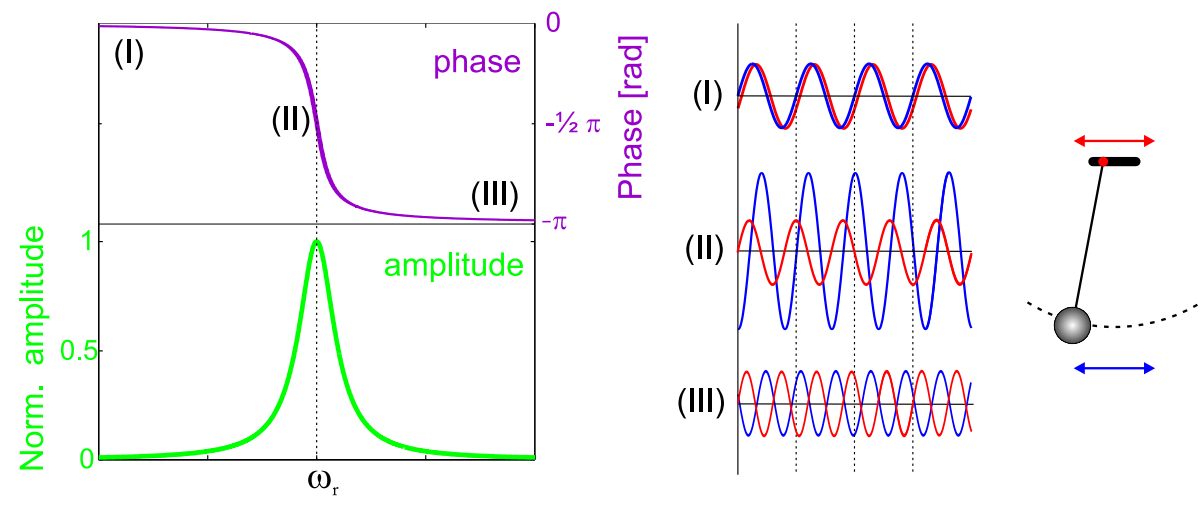

Driving frequency $\omega$

Figure 1.6: Amplitude and phase of a vibrational resonance.

ving force and the molecular response is called the "phase". The phase of a vibrational resonance can be illustrated by swinging a pendulum. The response of the pendulum, with a resonance frequency $\omega_{r}$, has three different regimes depending on the driving frequency $\omega$. These different regimes can be seen in figure 1.6 and are described by:

(I) $\omega<<\omega_{r}$; weak response, in phase $\left(0^{\circ}\right)$ with the driving force.

(II) $\omega \approx \omega_{r}$; strong response, $90^{\circ}$ out of phase with the driving force.

(III) $\omega>>\omega_{r}$; weak response, in counter-phase $\left(180^{\circ}\right)$ with the driving force.

This phase delay signifies resonant behavior.

\subsection{Thesis overview}

This thesis demonstrates a new CARS technique based on the detection of the phase of the CARS signal. We call this technique "Vibrational Phase Contrast CARS" or in short "VPC-CARS", and it can be seen as a vibrational extension of the linear (refractive index) phase contrast microscopy introduced by Zernike in 1933. The phase detection allows for a rejection of the non-resonant background signal, without reduction of the resonant signal.

In several collaborations with other research groups, CARS microscopy is used to answer questions in biology and pharmacology. 
Chapter 2 treats the theory behind the non-resonant background, as well as the origin and advances made in reducing this background in CARS spectroscopy and microscopy.

Chapter 3 presents heterodyne CARS detection to obtain the CARS amplitude and phase. A novel cascaded phase preserving chain is presented that offers shot noise limited detection of the CARS signals. Demonstrations are presented of spectroscopy and microscopy.

Chapter 4 shows an extension of the heterodyne CARS setup by measuring not only the heterodyne phase, but also a reference phase. The subtraction of both phases gives the pure vibrational phase of the molecules in the focal volume. This can be used to obtain background free images. Furthermore a third order cascaded phase-preserving chain is proposed and the VPC-CARS is explored further for compound analysis in the complex plane.

Chapter 5 demonstrates the capability of CARS microscopy as a multiphoton nonlinear imaging tool for biology and pharmacology. Studies on the dissolution behavior from tablets and bone formation in cells are presented.

Chapter 6 shows a variety of images obtained with CARS microscopy. The images are presented as microscale artistic images. 


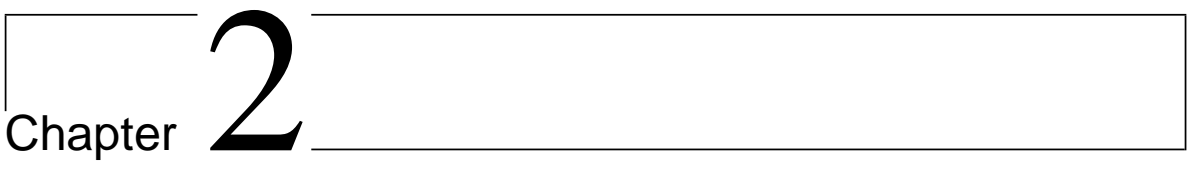

\section{The CARS process}

Coherent anti-Stokes Raman scattering (CARS) is a nonlinear optical process that addresses the intrinsic vibrational resonances of molecules and can be used to obtain chemically specific imaging of a sample. In this chapter the nonlinear susceptibility, and its relationship to the resonant and non-resonant contributions to the CARS signal will be discussed. The non-resonant contribution can overwhelm the resonant contribution. To reduce or cancel the non-resonant contribution, several techniques have been developed and will be discussed briefly. In the last part of this chapter an overview is given of the various setups and detection schemes used throughout the thesis. 


\subsection{Coherent anti-Stokes Raman scattering}

\subsubsection{Nonlinear optics}

When a wave is incident on a medium, the electric field of the incident wave causes the dipoles in the medium to oscillate. When this electric field is weak, the atoms in the medium behave like harmonic dipoles oscillating at the frequency of the excitation field. This behavior yields a linear dependence of the polarization on the electric field and describes the refractive index of a material. The oscillation of the dipoles becomes less linear when the incident field gets stronger. The dipole motion can be expended in powers of the electric field, where the higher orders describe different frequencies. The motion induces a field and energy is transferred from the driving frequency component to others. The relation between the polarization $\mathbf{P}$ and the electric field $\mathbf{E}$ can be written as [14]

$$
\mathbf{P}=\epsilon_{\circ}\left(\chi^{(1)} \cdot \mathbf{E}+\chi^{(2)} \cdot \mathbf{E}^{2}+\chi^{(3)} \cdot \mathbf{E}^{3}+\ldots\right),
$$

where $\chi^{(1)}$ is the linear susceptibility and $\chi^{(2)}, \chi^{(3)}, \ldots$ are the higher order (nonlinear) susceptibilities of the medium. The linear susceptibility is responsible for the linear optical properties of the medium such as reflection, dispersion, absorption. The second-order susceptibility $\chi^{(2)}$ gives rise to nonlinear optical processes such as second-harmonic generation (SHG) and optical parametric amplification (OPA). The third-order susceptibility $\chi^{(3)}$ is responsible for the nonlinear optical processes such as Raman scattering, CARS and third harmonic generation (THG). In general the magnitude of the susceptibility decreases rapidly with increasing order of nonlinearity [15]. Since the invention of the laser electric fields of such sufficient magnitude can be generated that the second and higher order terms become significant.

\subsubsection{The third-order nonlinear susceptibility}

In the CARS process, the pump, Stokes and probe fields drive the oscillations of the molecules in the focal volume coherently. The collective motion of the dipoles generates a macroscopic third-order polarization at the anti-Stokes frequency. The pump and probe are often taken to have the same frequency and amplitude and to originate from the same laser source. The third order polarization can then be expressed as

$$
P^{(3)}\left(\omega_{p}, \omega_{s} ; \omega_{c}\right)=\chi^{(3)}\left(\omega_{p}-\omega_{s}\right) E_{p}^{2}\left(\omega_{p}\right) E_{s}\left(\omega_{s}\right) .
$$


By tuning the difference frequency between the pump and Stokes to a vibrational resonance or Raman mode, the third-order polarization increases dramatically.

The frequency dependence of a vibrational resonance can be described as a damped driven harmonic oscillator. For a single vibrational resonance the frequency dependence can be approximated by a complex Lorentzian function [16]

$$
\chi_{r}^{(3)}=\frac{A_{R} \Gamma_{R}}{\Omega_{R}-\omega-i \Gamma_{R}},
$$

where $A_{R}$ is the normalized strength of the vibrational mode $R, \Omega$ is the vibrational frequency, $\omega$ represents the difference frequency between the pump and the Stokes fields $\left(=\omega_{p}-\omega_{s}\right)$ and $\Gamma$ is half-bandwidth at half-maximum with the Raman line.

When describing multiple vibrational resonances, the $1 / \omega^{2}$ term that is discarded in the complex Lorentzian approximation cannot be neglected. This frequency dependence requires a small correction. In the case of a weak resonance close to a strong resonance these small corrections in the wings of the resonance result in a large relative mistake in the amount of non-resonant signal for the weak resonance. The frequency dependence of vibrational resonances can be described better by the solution of the damped harmonic oscillator directly [16]

$$
\chi_{r}^{(3)}=\sum_{R} \frac{A_{R} \Gamma_{R} \Omega_{R}}{\Omega_{R}^{2}-\omega^{2}-2 i \omega \Gamma_{R}} .
$$

This vibrationally resonant signal is not the only contribution to the anti-Stokes signal. In the absence of a vibrational resonance, the dipole still oscillates, resulting in a CARS response at the anti-Stokes frequency. This detuned part is the non-resonant contribution to the anti-Stokes frequency. A two-photon enhancement of this non-resonant contribution is obtained when $2 \omega_{p}$ is close to the frequency of an electronically excited state. To avoid two-photon enhanced electronic contributions, near-infrared wavelengths are preferred [17].

The third-order nonlinear susceptibility can be split in a resonant $\left[\chi_{r}^{(3)}\right]$ part and a non-resonant $\left[\chi_{n r}^{(3)}\right]$ part

$$
\chi^{(3)}=\chi_{r}^{(3)}+\chi_{n r}^{(3)} .
$$


The $\chi^{(3)}$ tensor consists of 81 separate elements, which reduces due to symmetry into 21 different non-zero elements, where four of these elements contribute to the resonant CARS signal and the others are electronic non-resonant contributions. The non-resonant contributions show negligible variation upon tuning the frequency difference between pump and Stokes fields. The non-resonant susceptibility is therefore frequency independent and real $[18,16]$.

Since the total CARS signal is proportional to the square modulus of the nonlinear susceptibility [19], the intensity of the CARS signal can be written as

$$
\begin{aligned}
I_{C A R S}(\omega) & \propto\left|\chi^{(3)}(\omega)\right|^{2} \\
& =\underbrace{\left|\chi_{r}^{(3)}(\omega)\right|^{2}}_{(I)}+\underbrace{\left|\chi_{n r}^{(3)}\right|^{2}}_{(I I)}+\underbrace{2 \chi_{n r}^{(3)} \operatorname{Re}\left[\chi_{r}^{(3)}(\omega)\right]}_{(I I I)},
\end{aligned}
$$

where (I) is the frequency dependent resonant contribution, (II) is the frequency independent non-resonant contribution and (III) is a mixing term. The individual contributions to the CARS signal can be seen in figure 2.1(c). The non-resonant contribution gives a background offset to the signal, whereas the mixing term causes a peak shift to the lower wavenumber side of the spectra and characteristic dip in the signal on the higher wavenumber side, see figure 2.1(d).

The phase of the total CARS signal is determined by the phase of the non-resonant signal and mixing term, where the non-resonant is purely real. The CARS signal phase deviates from zero up to $\pi / 2$ around the resonance, limited by the non-resonant background. After the resonance, the phase returns to zero, see figure 2.1(b).

The mixing of the resonant and non-resonant contributions results in a fano-line profile, see figure 2.1(d). Within this line shape three regions can be distinguished: the on-resonance (f), negative contrast (g) and off-resonance (e). Images taken at these locations in the spectrum, are shown in figure 2.1. The combination of multiple resonances close to each other results in complex vibrational resonance line shapes. Interpretation of CARS spectra, with their quadratic dependence on the number of oscillators and mixing between the resonant and non-resonant signals, is not as straightforward as for Raman spectra. For weak resonances or low concentrations of molecules, the non-resonant background 

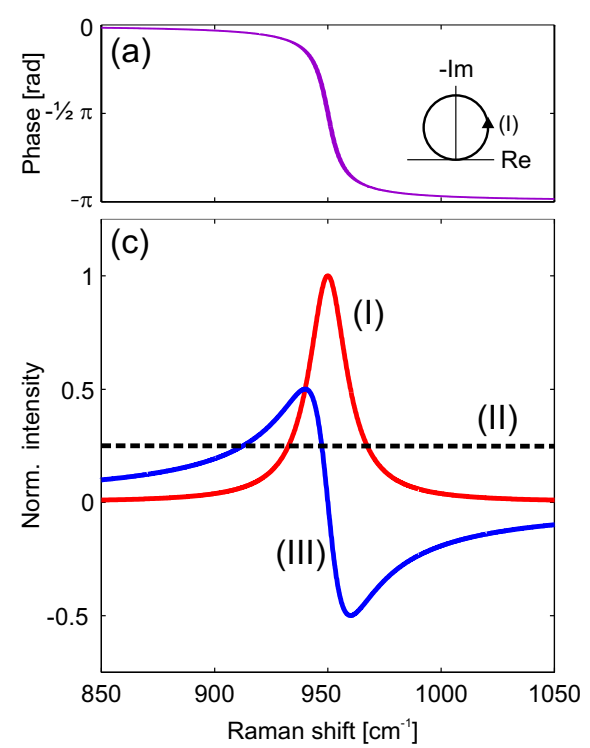
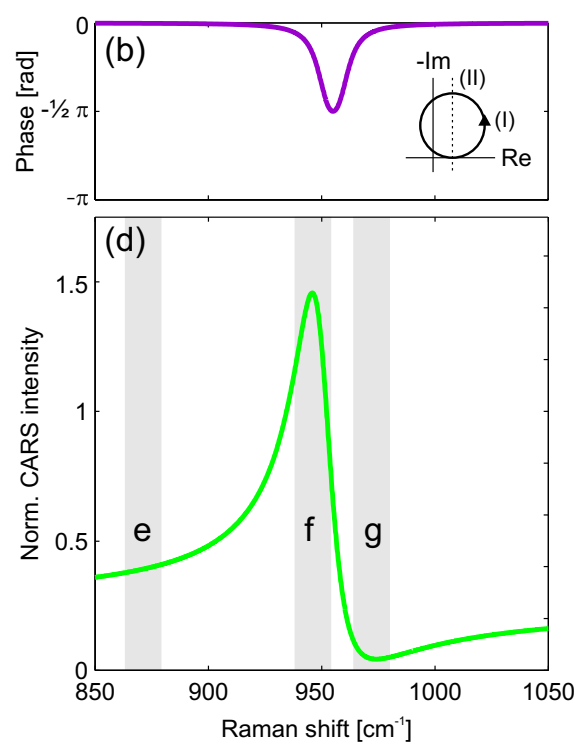
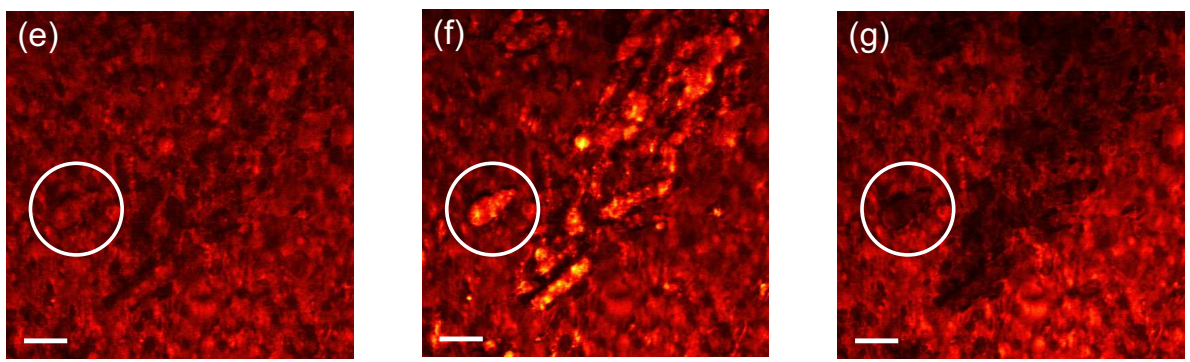

Figure 2.1: Contributions to the CARS spectrum. (c) The individual components; the resonant contribution (I), non-resonant contribution (II), and the mixing term (III). (d) Shows the total CARS spectrum. (eg) Shows an image at the different positions in the spectrum, indicated in (d): off-resonant (e), on-resonant ( $f$ ) and negative contrast $(g)$ image. (a) and (b) show the phase of the resonant contribution and the phase of the CARS signal respectively. The insets show the trajectory of the amplitude and phase through the complex plane.

may overwhelm the resonant contribution to the CARS signal, so suppression or rejection of the non-resonant contributions becomes essential. 


\subsubsection{CARS and Raman}

Even through the generation of the CARS signal is based on the Raman response of molecules, there are distinct differences between CARS and Raman scattering. In CARS, the vibration resonance is driven by the difference frequency of the pump and Stokes frequencies. Raman scattering is a spontaneous process. For Raman scattering the incoming field can originate from a single continuous wave laser, where CARS requires a more complex setup with pulsed sources to obtain the necessary high intensities. Furthermore, CARS requires tuneability of one of the input frequencies to set the difference frequency of interest. At the detection side, Raman scattering signals are red-shifted compared to the incoming field and may suffers from overlap with (auto)fluorescence from the sample. The CARS signal is blue-shifted, and thus free from one-photon auto-fluorescence, but low numbers of molecules or weak vibrational resonances interfere with the non-resonant contribution. The CARS signal has a coherent build-up, which results in a signal dependence which depends on the square of the number of oscillators as opposed to a linear dependence for Raman scattering.

An added restriction to the coherent build-up is that the beams in the CARS process have to fulfill the phase-matching condition. The interacting waves have to be aligned with respect to each other to minimize the wave vector mismatch. One common used phase-matched geometry is folded BoxCARS [20]. A tight focusing configuration relaxes the phase-matching condition due to the fact that the interaction length is kept relatively short. The coherent build-up ensures that the CARS signal is generated in one direction, which allows for higher collection efficiencies, whereas the Raman signal is emitted in all directions.

The dependence of the Raman signal intensity on the third-order nonlinear susceptibility is given by $[21]$

$$
I_{\text {Raman }} \propto \operatorname{Im}\left[\chi_{r}^{(3)}\right] .
$$

Raman scattering relates only to the imaginary part of the third-order nonlinear susceptibility and is only sensitive to the resonant part of the material response.

The ability of Raman spectroscopy to record a complete vibrational spectrum of a point in the sample in less than a second is superb when compared to CARS spectroscopy. In CARS spectroscopy the use 
of broadband femtosecond pulses can cover large parts of the spectrum $[22,23]$, but can not compete with the spectroscopic and background-free capabilities of Raman spectroscopy in the absence of auto-fluorescence. On the other hand, to record an image of 256x256 pixels with Raman microscopy can easily take up to an hour or more, whereas CARS microscopy can reach video rate imaging speeds (pixel dwell time of $0.16 \mu \mathrm{s}$ ) at a single vibrational resonance of interest [24]. Due to the nonlinear power dependence in the CARS process, the CARS signals are generated in the focal volume, where the powers are sufficiently high, providing a inherently confocality. This allows for high three-dimensional resolution without a confocal pinhole.

\subsection{The non-resonant background}

Depending on the ratio of resonant to non-resonant molecules in the focal volume, the non-resonant signal can overwhelm a small resonant contribution. Samples that contain a lot of water, such as cells, give rise to a significant non-resonant signal, observed as a background over the entire image. The intensity differences in such images are not based on chemical selectivity alone, but contain interferences between the resonant and non-resonant signal. In the last decade several methods have been used to reduce the non-resonant background.

\section{Phase matching based}

One commonly used method to remove the background is epi-CARS [25], where the CARS signal is detected in the backward direction. This method does not really offer discrimination between resonant and nonresonant CARS signal, but between large and small objects in the focal volume, based on phase matching. Small resonant objects give rise to CARS signal in both the forward and backward direction, whereas the surrounding bulk medium (e.g. non-resonant water) causes only CARS signal in the forward direction. The non-resonant signal from the small object itself is not reduced in epi-CARS. The technique is especially suitable for transparent samples such as single cells, see figure 2.2. When imaging highly scattering media such as tissue the epi-CARS signal will be overwhelmed by backscattering of forward-generated CARS signals. Another technique based on the phase-matching condition is wide field CARS [26], where the variations in refractive index changes in the sample are used to fulfill the phase-matching conditions. This technique requires special treatment of the sample. 

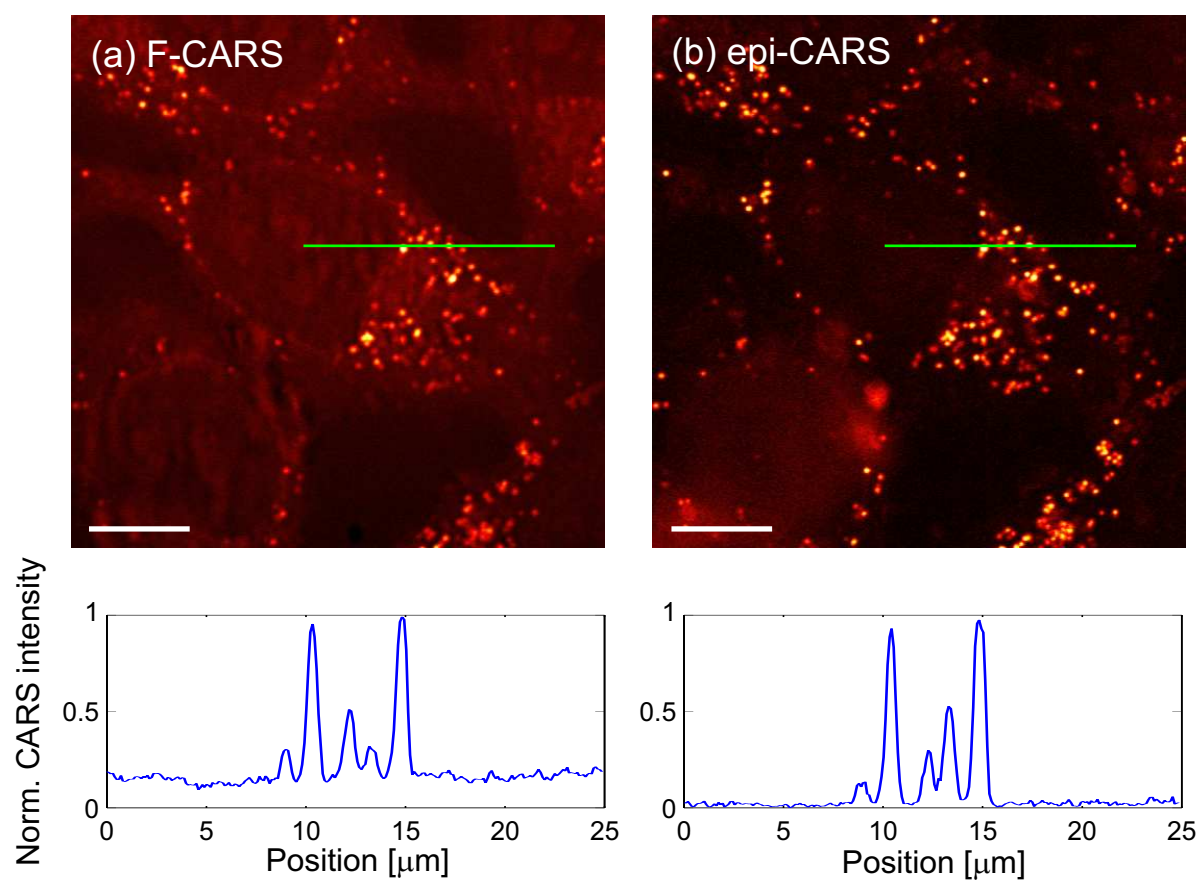

Figure 2.2: HeLa cells imaged at the vibrational resonance of $2845 \mathrm{~cm}^{-1}$. (a) Forward CARS detection shows a large non-resonant background over the complete image and (b) epi-CARS detection shows better contrast of the small features. The CARS intensity profile on the location of the green line are shown below the images. The scale bar is $10 \mu \mathrm{m}$.

\section{Amplitude based}

As shown in figure 2.1(d), the CARS spectrum has a very distinctive line shape. The difference between the off-resonant (e) or negative contrast (g) and on-resonant (f) point can be used to remove the background from the image, when assuming the non-resonant background has a constant intensity between the on- and off-resonance point. Dual pump CARS $[27,28]$ measures at two vibrational resonances simultaneously on two detectors. Two optical parametric oscillators (OPO) can be used or one OPO that runs simultaneously at two different wavelengths by proper positioning of the Lyot filter, where one wavelength is set to be on-resonance and the other is set to be off-resonance. This Lyot filter technique requires high stability, and the difference between the wavelengths is very hard to obtain. 


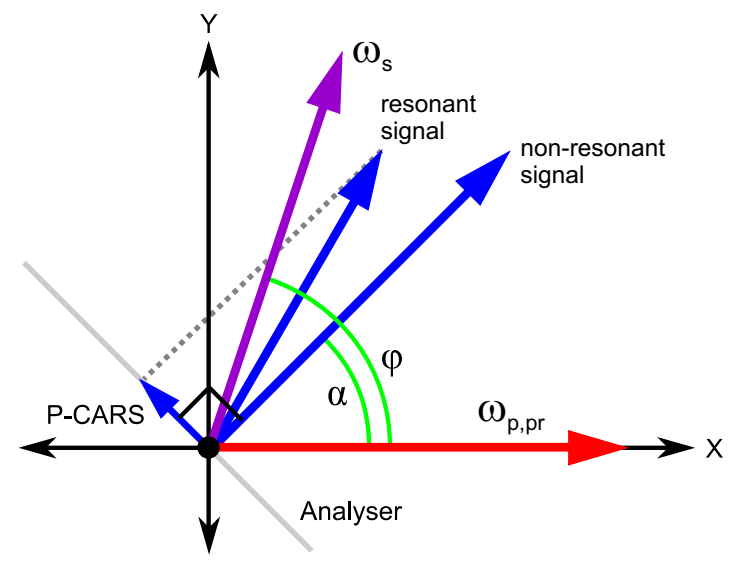

Figure 2.3: Diagram showing the polarizations of the various beams in the P-CARS configuration.

Frequency Modulation (FM)CARS [29] exploits the same spectral differences between the resonant and non-resonant signal. Here two OPO's are used to set the wavelengths accurate to the maximum and minimum of the CARS spectrum. The amplitude modulated CARS signal is detected on a detector. Both techniques require a constant nonresonant background for faithful reproduction of the resonant amplitude.

Stimulated Raman scattering (SRS) $[30,31]$ is a technique that enjoys renewed interest $[32,33,34]$. This technique is inherently background free and relies on stimulating the Raman scattering by adding a Stokes wavelength on the desired frequency difference between the pump and Stokes. Applying a modulation on the pump or Stokes beam results in a small modulation on a large background in the other beam. This technique has been demonstrated successfully for background free and fast imaging of single vibrational resonances.

\section{Time based}

Time-resolved CARS [35] exploits the differences in dephasing time between resonant and non-resonant components. The non-resonant component is rejected by delaying the probe beam.

\section{Polarization based}

The resonant and non-resonant contributions generally experience a different dependence on the polarization of the input frequencies. Polari- 


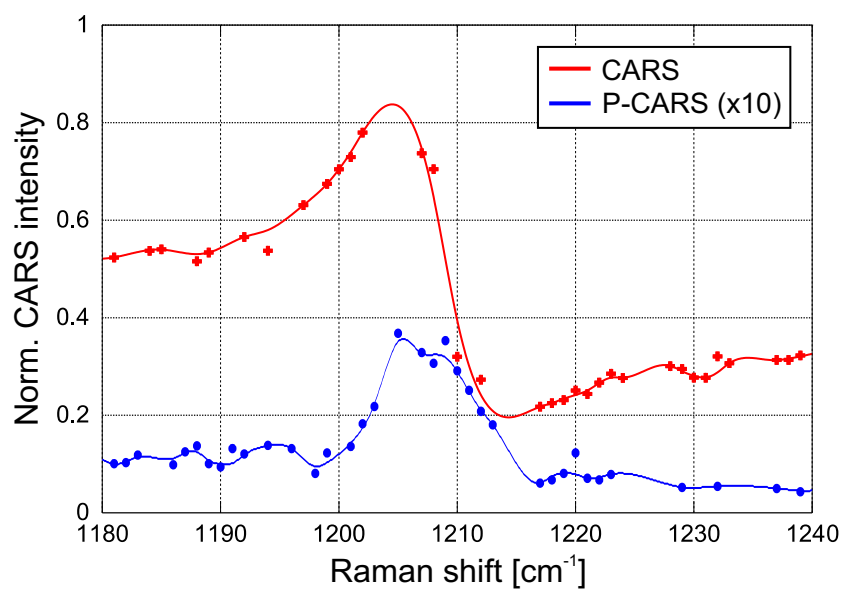

Figure 2.4: Measurement on toluene around the vibrational resonance of $1207 \mathrm{~cm}^{-1}$, showing the CARS intensity and the background free PCARS intensity (scaled up by a factor of 10).

zation CARS $[36,37]$ exploits this difference to remove the non-resonant signal, but unfortunately often also rejects a large part of the resonant signal. An early description of polarization CARS (P-CARS) was given by Ahkmanov in 1977 [38] and is sketched in figure 2.3. Two input beams at frequencies $\omega_{p}$ (pump/probe) and $\omega_{s}$ (Stokes) propagate colinearly along the $z$-axis. The polarization of the pump beam lies along the $x$-axis, the polarization of the Stokes beams is at an angle $\varphi$. For a homogeneous sample, the non-resonant component of the $\chi^{(3)}$ tensor is real and shows no preferred direction or depolarization. The resonant contribution is rotated by an amount that depends on the difference in the induced polarization along different axes; the depolarization ratio. The resonant and non-resonant contributions to $\chi^{(3)}$ are then separated in angle. Taking $\alpha$ as the angle between the polarizations of the pump beam and the non-resonant component of $\chi^{(3)}$, it can be seen that the ratio of the resonant to non-resonant contributions is maximized when $\alpha=45^{\circ}$, which occurs when $\varphi=71.6^{\circ}$ [37]. The non-resonant component of $\chi^{(3)}$ can be rejected with an orthogonal analyzer (polarizer), leaving only a portion of the differently polarized resonant signal. Figure 2.4 gives a demonstration of the difference in spectrum obtained with and without P-CARS. In this figure the characteristic dispersive CARS spectrum is shown of toluene around $1200 \mathrm{~cm}^{-1}$ and with P-CARS the signal reduces dramatically, but the Raman resonance line shape is retrieved. 


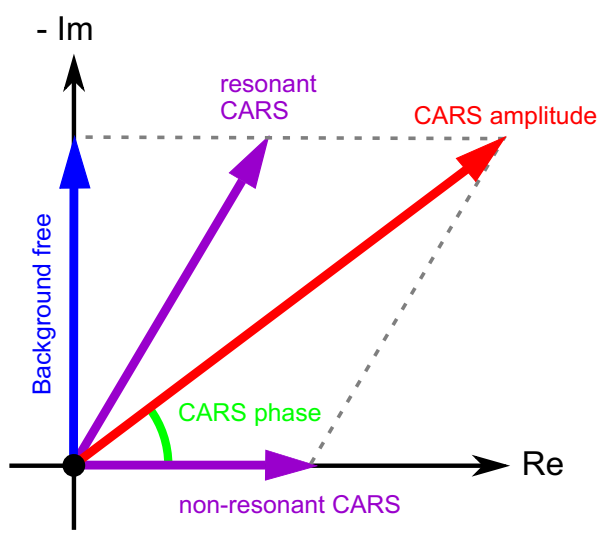

Figure 2.5: Complex plane, where the CARS amplitude is constructed from the resonant and non-resonant contributions to the third-order nonlinear susceptibility. The projection of the CARS amplitude onto the imaginary axis results in a background free signal.

\section{Phase based}

Interferometric CARS [39, 40] achieves rejection of the non-resonant CARS signal without rejection of resonant signal via direct detection of the amplitude and phase of the CARS signal. Furthermore it allows for interferometric amplification of the signal. In figure 2.1(b) the phase profile is shown for a single resonance in the CARS spectrum. Figure 2.5 shows how the CARS signal is created by the addition of the resonant and non-resonant contributions of the third-order nonlinear susceptibility in the complex plane. The non-resonant contribution is strictly real, whereas the phase of the resonant contribution is wavelength-dependent and complex, see section 2.1.2. The length of the vector in the complex plane is proportional to the CARS amplitude and the angle of the vector with the real axis represents the CARS phase. The projection of the CARS amplitude onto the imaginary axis gives a background free signal, without the real components. This background free signal is directly related to the Raman signal, see equation 2.7. Another advantage of measuring the amplitude of the CARS signal rather than the intensity is that the amplitude is proportional to the number of oscillators in the focal volume, which makes the decomposition of signals much easier and is advantageous at low numbers of oscillators.

Heterodyne CARS [41] mixes the CARS signal with a stable external reference signal. This avoids possible misinterpretation due to 


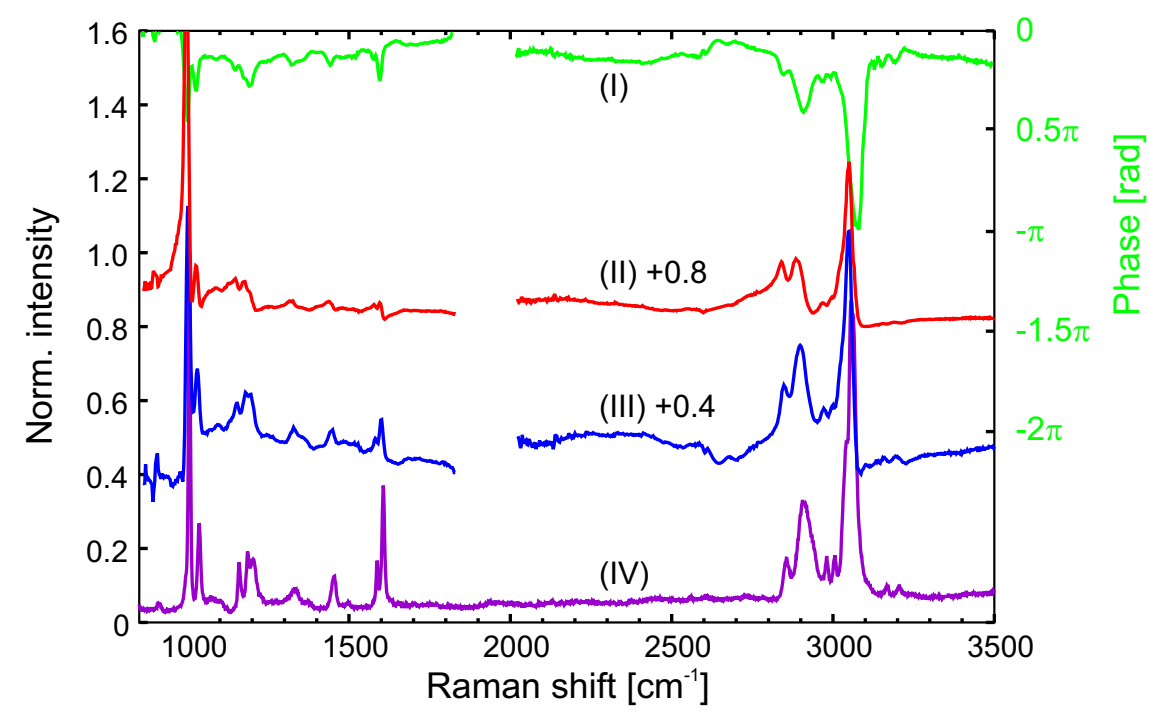

Figure 2.6: Resonant spectrum extracted from a CARS spectrum of polystyrene using the maximum entropy method. (II) Narrow band CARS intensity spectrum, (I) phase spectrum obtained with the maximum entropy method, (III) background free CARS spectrum by applying the phase spectrum on the CARS intensity spectrum and (IV) the Raman spectrum as comparison.

inhomogeneous distribution of the non-resonant background when that background is used as the local oscillator.

In multiplex CARS (M-CARS) spectroscopy, spectra are recorded over large parts of the vibrational spectrum [36, 42, 43]. In the fingerprint region the spectra are highly congested. Using the maximum entropy method $[44,45]$ on the measured CARS spectrum, the phase can be derived and a background free spectrum can be retrieved. Figure 2.6 shows the application of the maximum entropy method to a CARS spectrum of polystyrene. This spectrum is not measured using a broadband method, but by scanning a narrowband source. The line shapes in the processed spectrum resemble those in the spontaneous Raman spectrum better.

\section{Other phase based methods}

The spatial and spectral phase can also be exploited to reduce the nonresonant CARS signal as in spatial phase control CARS $[46,47]$ or spectral phase shaping CARS $[48,49,50]$. 
In this thesis heterodyne detection will be used to detect the amplitude and phase of the CARS signal to obtain background free images. The non-resonant signal will be canceled, where the resonant signal will not be reduced in strength but will be amplified by interferometric amplification. The detection of amplitude and phase is reproducible due to the use of a stable local oscillator.

\subsection{Experimental setup}

\subsubsection{The choice of laser source}

The development of nonlinear microscopy goes hand-in-hand with the development of new laser sources. Wavelength, pulse duration and repetition rate are the main parameters for CARS microscopy. The choice of the wavelengths for the generation of the CARS signal is important because it affects the amount of non-resonant CARS signal. A source in the near infrared reduces this effect and provides a better ratio of signal to non-resonant background. Furthermore, sample damage can occur due to multiphoton absorption in the sample. The use of wavelengths longer than $800 \mathrm{~nm}$ significantly decreases sample damage [51]. Another advantage of using near infrared wavelengths is that these wavelengths scatter less in tissue, allowing for deeper penetration [52]. However, the wavelength need to be kept shorter than $1.1 \mu \mathrm{m}$ to avoid absorption in water and ensure reasonable resolution.

Due to the cubic intensity dependence of the CARS signal, pulsed laser systems are necessary to obtain the high peak intensities needed. Vibrational resonances have a typical linewidth on the order of $10 \mathrm{~cm}^{-1}$. The resonant signal increases with the pulse spectral width, until the spectral width of the pulse becomes larger than the Raman line width. The ideal pulse spectral width corresponds to pulses of $\sim 3$ picosecond duration for a typical Raman band [53]. The repetition rate and pulse energy are related to the scanning speed and sample damage. Repetition rates in the range of $0.1-100 \mathrm{MHz}$ and picosecond pulse powers up to a few nJ are optimal [18].

The pulses for the pump/probe and Stokes need to have exactly the same repetition rate. A difference in repetition rate will result in a loss of temporal overlap in the sample and a loss of the CARS signal. To obtain the same repetition rate, synchronously pumped lasers or tightly 
synchronized lasers can be used. Some of these possibilities are:

- Two synchronously pumped dye lasers (pump and Stokes: 540-870 nm) [54].

- Two tightly synchronized Mode-locked Ti:Sapphire lasers (pump and Stokes: 710-920 nm, 3ps) [55].

- Intracavity-doubled synchronously-pumped optical parametric oscillator (pump: 780-920 nm, 5 ps; Stokes: 1064 nm, 7 ps) [24].

- Broadly tunable optical parametric oscillator using signal and idler (pump: 850-1020 nm, Stokes 1100-1350 nm, 7.5 ps) [56].

In this thesis several configurations of a laser source and an optical parametric oscillator $(\mathrm{OPO})$ are used:

- Spectra-Physics Vanguard 2000-HM532, Nd:YVO $4,80 \mathrm{MHz}$, $2 \mathrm{~W}, \sim 12 \mathrm{ps} @ 532 \mathrm{~nm}, 1 \mathrm{~W}, \sim 15 \mathrm{ps} @ 1064 \mathrm{~nm}$.

- Coherent Paladin, Nd: $\mathrm{YVO}_{4}, 80 \mathrm{MHz}$, $12 \mathrm{~W}, \sim 11 \mathrm{ps} @ 532 \mathrm{~nm}, 1.5 \mathrm{~W}, \sim 16 \mathrm{ps} @ 1064 \mathrm{~nm}$.

Three OPO's are used, each with their own specifications. (s+i) and $(\mathrm{s} / \mathrm{i}+\mathrm{f})$ indicate the vibrational frequencies covered:

- Home-built OPO, LBO (Brewster angeled) [57, 58] signal: $740-930 \mathrm{~nm}$, idler: 1240-1890 s+i: $2710-8232 \mathrm{~cm}^{-1}, \mathrm{~s} / \mathrm{i}+\mathrm{f}: 1355-4301 \mathrm{~cm}^{-1}$

- Home-built OPO, PPLN:MgO [59] signal: 880-1040 nm, idler: 1090-1350 nm s+i: $436-3932 \mathrm{~cm}^{-1}, \mathrm{~s} / \mathrm{i}+\mathrm{f}: 218-1966 \mathrm{~cm}^{-1}$

- APE Levante Emerald, LBO signal: $690-990 \mathrm{~nm}$, idler: 1150-2300 nm s+i: $1407-10189 \mathrm{~cm}^{-1}, \mathrm{~s} / \mathrm{i}+\mathrm{f}: 703-5095 \mathrm{~cm}^{-1}$

The OPO is temperature tuned. Small wavelength changes $(10-15 \mathrm{~nm})$ can be obtained by rotation of the birefringent Lyot filter [60] within the cavity of the OPO. This selects wavelengths within the (temperature dependent) gain bandwidth of the OPO. 


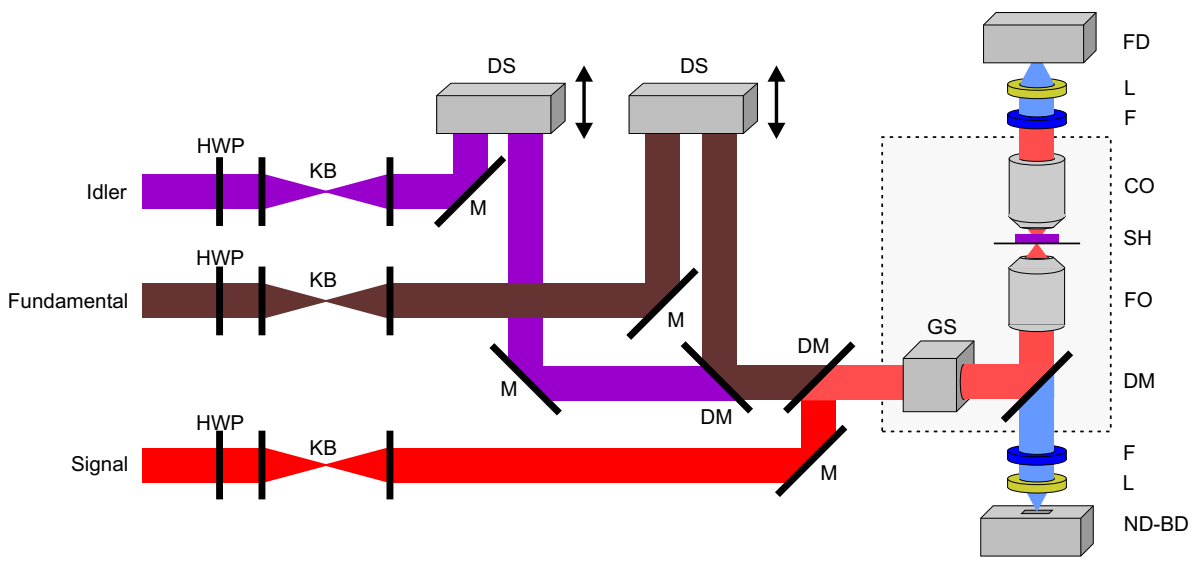

Figure 2.7: CARS setups. HWP: $\lambda / 2$ plate, KB: Keplerian beam expander, DS: Delay stage, M: Mirror, DM: Dichroic mirror, GS: Galvano scanner, FD: Forward detector, ND-BD: Non-descanned backward detector, FO: Focusing objective, CO: Collection objective, SH: Sample and sample holder, F: Filters and L: Lens

\subsubsection{Microscopy setup}

The heart of the CARS setup is the microscope. In this thesis we use an Olympus IX71 inverted microscopy with FluoView 300 a beam scanning module. The fundamental of the laser and the signal and idler from the OPO each pass a $\lambda / 2$ plate and a Keplerian beam expander to control the polarization, size and divergence of the beams before entering the microscope. The fundamental and idler beams pass delay lines to control temporal beam overlap. These two beams are spatially overlapped on a dichroic mirror (Chroma z1064dcrb-nb) and then the signal is added (Chroma 1000dcxr-xt). The optics inside the microscope are coated for optimal near-infrared transmission. The beams are scanned by two galvano mirrors. Before the objective the beams pass a filter wheel with dichroics for backward CARS. Three objective lenses are used: 0.6 NA 20x air, 0.9 NA 40x air and 1.2 NA 60x water immersion. A $0.55 \mathrm{NA}$ collimation lens collects the CARS signal and mirrors direct the signal to the detector. Bandpass filters are placed in front of the detector to block the input wavelengths. Figure 2.7 shows a schematic of the different beam paths towards and inside of the microscope. The typical resolution of the $1.2 \mathrm{NA}$ lens is $\sim 0.3 \mu \mathrm{m}$ in the lateral direction and $\sim 0.8 \mu \mathrm{m}$ in the axial direction [18]. 
Figure 2.8 shows the experimental setup for different CARS microscopy types. Note that the epi-CARS signal can be detected with the non-descanned backward detector as well as the descanned backward detector. The backward CARS detector is placed as close as possible to the objective lens to collect most of the backward scattering CARS signal. 


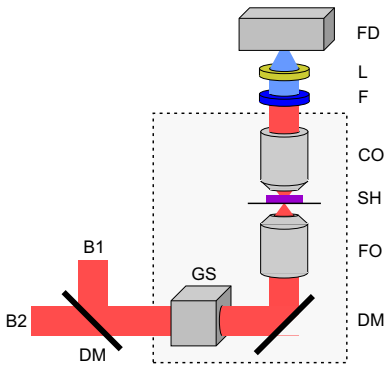

(a) Forward CARS (F-CARS)

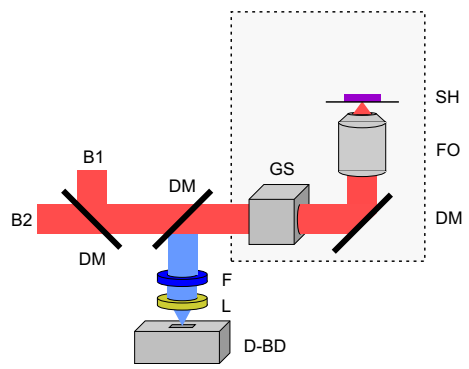

(c) Epi CARS (E-CARS)

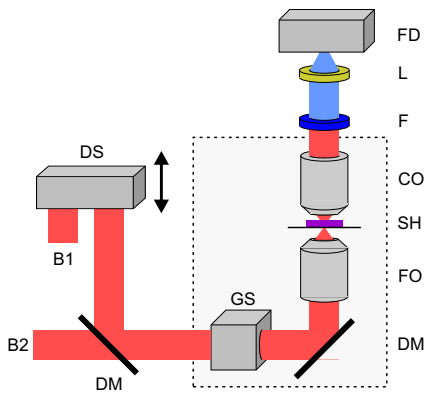

(e) Time resolved CARS

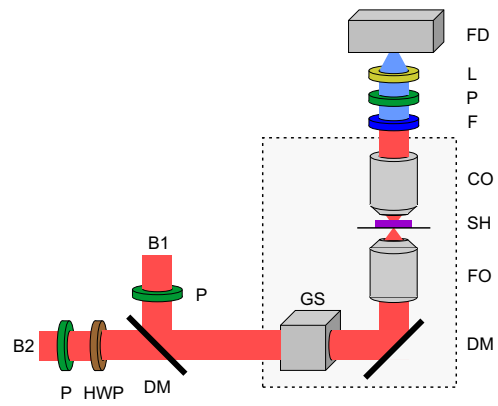

(b) Polarization CARS (P-CARS)

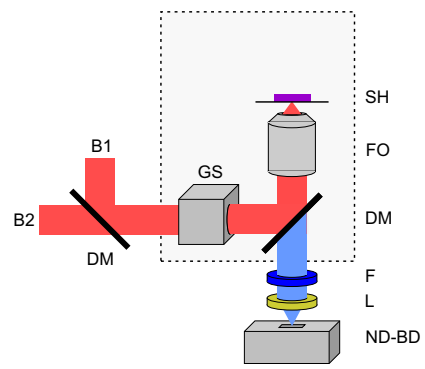

(d) Backward CARS (B-CARS)

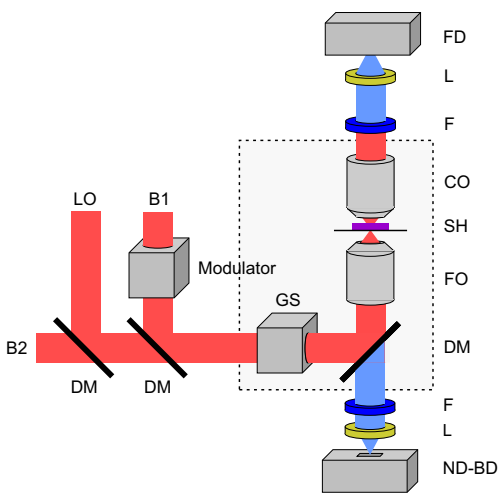

(f) Heterodyne CARS (H-CARS)

Figure 2.8: Different CARS setups. B1: pump, B2: Stokes, LO: Local oscillator, DM: Dichroic mirror, GS: Galvano scanner, FD: Forward detector, D-BD: Descanned backward detector, ND-BD: Non-descanned backward detector, FO: Focusing objective, CO: Collection objective, SH: Sample and holder, P: Polarizer, HWP: $\lambda / 2$ plate, F: Filters and L: Lens 
Chapter 2 


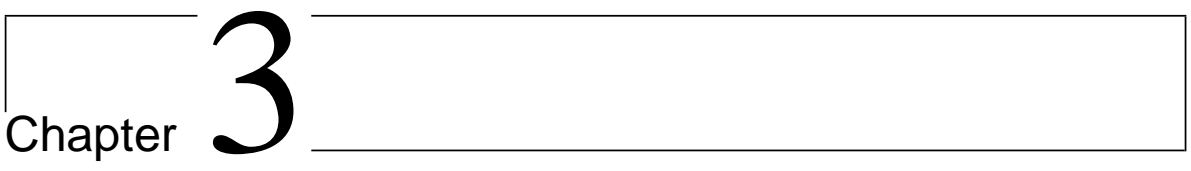

\section{Heterodyne CARS}

This chapter describes improvements in CARS sensitivity and selectivity by using heterodyne techniques. The improvements are obtained by interfering the weak CARS signal with a well controlled and stable local oscillator field. The local oscillator field is created by a cascaded phase preserving chain. The setup is inherently jitter-free and makes use of every single wavelength that is generated. The sensitivity is improved by more than 3 orders of magnitude for detection with a photodiode operating in the shot noise window of the local oscillator. Selectivity is improved by rejecting the background based on the phase of the CARS signal. In this chapter heterodyne detection, shot noise limited detection and the improvement of sensitivity and selectivity will be treated theoretically as well as experimentally. 


\subsection{Introduction}

Due to the coherent nature of the CARS process, interference methods can be applied to improve the sensitivity and the selectivity of CARS. Heterodyne interferometric CARS detection can reach the highest sensitivity limit by detecting at the shot noise limit. Furthermore the selectivity can be enhanced by detecting the vibrational phase of the molecules in the focal volume. This phase gives the possibility to separate the resonant, wavelength-dependent CARS signal $\chi_{R}^{(3)}(\omega)$ from the non-resonant wavelength-independent, CARS signal $\chi_{N R}^{(3)}$

$$
I_{C A R S}(\omega) \propto\left|\chi_{R}^{(3)}(\omega)+\chi_{N R}^{(3)}\right|^{2} .
$$

The main difference between the resonant and the non-resonant signals is the vibrational phase that is added to the resonant signal. Detection of this phase of the molecules gives the possibilities to obtain background (or non-resonant) free spectra and images. Due to the high sensitivity and selectivity obtained by heterodyne CARS, background free spectra and images can be recorded, revealing details normally hidden in the background.

\subsection{Heterodyne detection}

\subsubsection{Interferometric detection}

Interferometric detection mixes a reference field, the so-called Local Oscillator (LO) field, with the generated CARS field at the anti-Stokes (AS) frequency. The total intensity on the detector can be expressed as

$$
I_{\text {detector }}=\left|E_{L O}\right|^{2}+\left|E_{A S}\right|^{2}+2 E_{L O} E_{A S} .
$$

The CARS field is proportional to the excitation field consisting of the resonant and non-resonant vibrational response $\chi^{(3)}$,

$$
E_{A S} \propto E_{E x}\left[\chi_{R}^{(3)}+\chi_{N R}^{(3)}\right],
$$

where the excitation field is created in the CARS process by

$$
E_{E x}=E_{\text {Pump }}^{2} E_{\text {Stokes }}
$$

and $\chi^{(3)}$ can be expressed as

$$
\chi^{(3)}=\left\{\left[\chi_{N R}^{(3)}+\operatorname{Re}\left(\chi_{R}^{(3)}\right)\right] \cos \phi_{\chi}+\left[\operatorname{Im}\left(\chi_{R}^{(3)}\right)\right] \sin \phi_{\chi}\right\},
$$


where $\phi_{\chi}$ is the phase difference between the total CARS field and the LO field. This is also the phase difference between the (purely real) non-resonant and the (complex) resonant part.

For homodyne interferometric detection, a well-controlled and stable local oscillator is required to interfere with the generated CARS field. The requirements of the local oscillator are that it is phase and wavelength locked to the generated CARS signal. It has been demonstrated that the local oscillator can be created in bulk media [40,41] adjacent to the CARS setup and combined with the pump and Stokes beams either before or after the sample. The generated CARS signal in the sample can directly interfere with the colinear local oscillator signal, for interferometric detection. It has also been shown that the non-resonant signal created in the CARS process can be used as the local oscillator to amplify the resonant signal [61]; however, note that the amount of amplification is heavily dependent on amount of non-resonant signal created in the sample.

To detect only the interference term without the DC-offset terms of the local oscillator and CARS intensities, heterodyne detection can be applied. For heterodyne detection the local oscillator field is shifted in frequency with respect to the CARS field, causing a beating on the interference signal on the detector at the shifted frequency. This interferometric signal can be detected by a lock-in amplifier on the beating frequency. The lock-in amplifier gives the amplitude of the interferometric signal, and also gives the phase $(\phi)$ between the CARS field and the local oscillator field, giving the possibility to detect the phase of the molecules.

\subsubsection{Cascaded phase preserving chain}

To avoid the complexity and instability of creating the separate local oscillator in a bulk medium, a novel cascaded phase preserving chain is used. This cascaded phase-preserving chain (PPC) is presented in figure 3.1. Here the energy diagram of the wavelengths involved in creating the CARS signal are compared to the wavelengths employed in the optical parametric oscillator (OPO). The fundamental of the laser source $(1064 \mathrm{~nm})$ is partially frequency doubled to $532 \mathrm{~nm}$ (SHG). This doubling is phase coherent [16], where the phase of the $532 \mathrm{~nm}$ is given by the addition of the phases of the two fundamental photons that make up the $532 \mathrm{~nm}$. The $532 \mathrm{~nm}$ synchronously pumps an OPO generating 


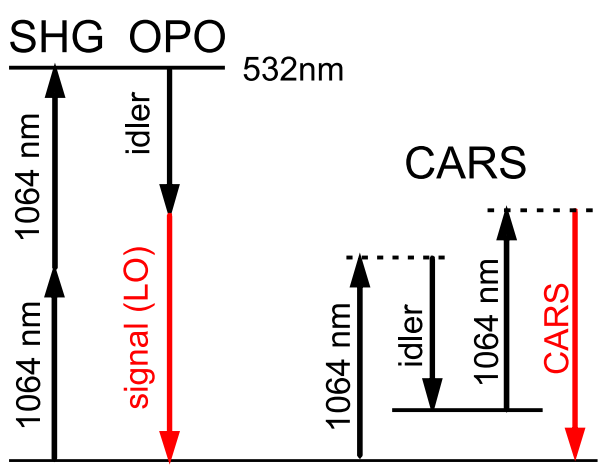

\begin{tabular}{|l|l|l|}
\hline Process & Wavelength relation & Phase relation \\
\hline 1. SHG & $2 \cdot \omega_{1064}=\omega_{532}$ & $2 \cdot \phi_{1064}=\phi_{532}$ \\
2. OPO & $\omega_{532}=\omega_{\text {signal }}+\omega_{\text {idler }}$ & $\phi_{532}=\phi_{\text {signal }}+\phi_{\text {idler }}$ \\
3. CARS & $\omega_{C A R S}=2 \cdot \omega_{1064}-\omega_{\text {idler }}$ & $\phi_{C A R S}=2 \cdot \phi_{1064}-\phi_{\text {idler }}+\phi_{\chi^{3}}$ \\
4. PPC & $\omega_{C A R S}=\omega_{\text {signal }}$ & $\phi_{C A R S}=\phi_{\text {signal }}+\phi_{\chi^{3}}$ \\
\hline
\end{tabular}

Figure 3.1: Schemetic of the energy diagram showing the optical chain for the phase-preserved generation of the wavelengths for the CARS process and local oscillator (LO). The table shows the wavelength and phase relations of the individual stages in the optical chain.

the signal and idler wavelengths. The phases of the signal and idler are independent, but the sum of their phases is locked to the phase of the pump. The freedom for the phase of the signal ensures that the resonating signal has no phase restrictions and projects all phase errors onto the non-resonating idler, ensuring smooth operation.

The idler wavelength is combined with the fundamental to generate a CARS (or anti-Stokes) signal, where the fundamental acts as the pump and probe and the idler as the Stokes wavelength. The phase of the OPO signal is given by twice the fundamental phase minus the idler phase. The generated CARS wavelength is equal to the signal wavelength from the OPO. Moreover, the phase of the resonant CARS signal is determined as twice the fundamental phase minus the idler phase, plus the phase of the vibrational response $\left(\chi^{(3)}\right)$. This phase of the vibrational response is constant for a fixed wavelength. The phase of the OPO signal is therefore locked to the phase of the CARS signal except for path-length variations between the point of creation and the point where they are combined. This phase preservation means that the signal wavelength can interfere with the CARS signal in a predictable way and 
can thus be used for interferometric amplification and phase detection. Furthermore the setup is jitter-free, due to the use of a synchronously pumped OPO, and makes use of every wavelength that is generated.

\subsection{Shot noise limited detection}

Heterodyne detection can significantly improve the detection of small CARS signals. In this section a treatment of the noise of the detector will be given. The interference of the local oscillator with the CARS signal yields a total signal intensity on the detector of

$$
I_{\text {detector }}=L O+C A R S+\underbrace{2 \cdot \sqrt{L O \cdot C A R S}}_{H C A R S},
$$

where local oscillator (LO) and CARS signify intensities and HCARS, the interference term, refers to the Heterodyne CARS power. This interference term scales with the root of the local oscillator power and the interferometric gain can be defined as

$$
\frac{H C A R S}{C A R S}=2 \sqrt{\frac{L O}{C A R S}} .
$$

The noise in the optical signal is determined by the shot noise in the CARS signal itself and the shot noise introduced by the local oscillator. The introduced shot noise is the dominant term of the two (as $L O \gg$ $C A R S$ ) and scales with the root of the amount of photons

$$
\text { Shot noise }=\sqrt{\# \text { photons }} \text {, }
$$

just like the interference term and the interferometric gain. The interferometric amplification can thus be used to lift the signal above the detector noise without degrading the original signal-to-noise ratio as long as the local oscillator shot noise is the dominant noise term.

\subsubsection{Detectors}

The most commonly used detectors in CARS microscopy are photomultiplier tubes (PMTs), photodiodes or avalanche photo diodes (APDs). All three detectors have their advantages and disadvantages, as can be seen in table 3.1. A photon-counting APD generates an electrical pulse for each detected photon. The PMT and photodiode are connected to a transimpedance amplifier that contains an OPerational AMPlifier 


\begin{tabular}{|l|l|l|l|}
\hline & APD & PMT & Photodiode \\
\hline Detector noise & 5 & 100 & $100 \mathrm{k}$ \\
Sensitivity for NIR & $85 \%$ & $0.1-10 \%$ & $85 \%$ \\
Sensitive area & small & large & large \\
Cost & very high & high & low \\
Damage threshold & low & low & high \\
\hline
\end{tabular}

Table 3.1: The advantages and disadvantages of three commonly used detectors in CARS microscopy: avalanche photodiode (APD) in counting mode, photo-multiplier tube (PMT) and photodiode. Detector noise represents the amount of photons at $40 \mathrm{Khz}$; sensitivity for near-infra red (NIR) is the quantum efficiency at $900 \mathrm{~nm}$.

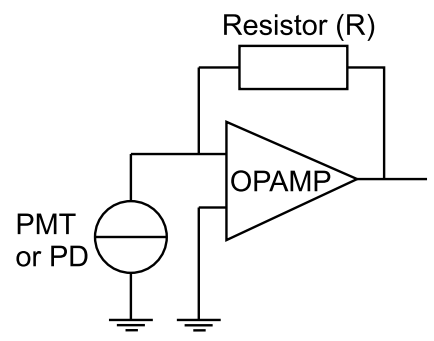

Figure 3.2: Transimpedance amplifier terminated at zero Ohm by an OPerational AMPlifier (OPAMP)

(OPAMP), as depicted in figure 3.2. The photodiode can be considered as a current source with a current proportional to the detected intensity, typically $0.6 \mathrm{~A} / \mathrm{W}$ at $900 \mathrm{~nm}$, which corresponds to a conversion of $\sim 85 \%$ electrons per photon. This conversion factor is called the quantum efficiency. PMTs tend to have a low quantum efficiency ( $10 \%$ to $0.1 \% \mathrm{QE}$ ) for wavelengths towards the near-infra red (NIR). APDs have the same QE as photodiodes (typically up to 85\%). For the detection of very low signals, therefore, APDs are the best. For practical applications photodiodes are favored because they are large, cheap and robust. APDs are expensive, small and fragile and suffer from a reduced dynamic range. The PMT is similar to the photodiode except that the dark current is lower when compared to a transimpedance amplifier of similar gain. At a gain of $10^{7}$ a PMT typically has a dark count noise of 100 $\mathrm{sec}^{-1}$, where a photodiode has $100 \mathrm{k} \mathrm{sec}^{-1}$. APDs have the lowest dark count rate, down to $5 \mathrm{sec}^{-1}$.

Considering user-friendliness (sensitive area and damage threshold), 
commercial interest (cost) and the application for CARS (detector noise and sensitivity for NIR) a photodiode scores very well, except for the detector noise. The dark counts of a photodiode are huge compared to the other two detectors. With heterodyne detection the generated CARS signal can be amplified with the local oscillator to lift it above the dark counts, so the photodiode is a nearly perfect detector for this experiment.

For a fair comparison between the different detectors and their noise characteristics, the detector efficiency should not be a factor. For this reason the noise of the detectors is compared in the Noise Equivalent Power (NEP), which is obtained by dividing the noise current of the detector by the detector sensitivity

$$
N E P=\frac{I_{\text {noise }}[A]}{\text { detector sensitivity }[A / W]} .
$$

The NEP as a function of local oscillator power on the detector is sketched in figure 3.3 and contains three different regions:

(1) At low powers the noise of the detector is determined by the most dominant noise source in the electronics. This noise source can come from the amplifier current noise (dark current noise and OPAMP noise), independent of $R$, or from the Johnson noise in the resistor $(\sqrt{4 k T R \cdot B W}$ where $B W$ represents the detection bandwidth in $\mathrm{Hz}$ ). Proper design of the transimpedance amplifier ensures that the noise is determined by the Johnson noise and that the detected voltage scales linearly with the transimpedance resistor $(R)$. By increasing the resistor to the point where it dominates the other noise sources, the signal-to-noise of the amplifier scales with $\sqrt{R}$. The maximum $R$ is limited by the required bandwidth of the amplifier, which scales as $1 / R$. A typical configuration yields a $1 \mathrm{MHz}$ bandwidth for $1 \mathrm{M} \Omega$ transimpedance. This Johnson noise will hereafter be referred to as the dark noise of the detector.

(2) At low levels of local oscillator on the detector the noise is dominated by the shot noise. This is the noise in the number of electrons in the detector and scales with the square root of the amount of photons on the detector, equation 3.8.

(3) At higher local oscillator powers, relative intensity noise (RIN) dominates. This scales directly with the number of photons of the local oscillator. More about the RIN can be found in section 3.3.2. 


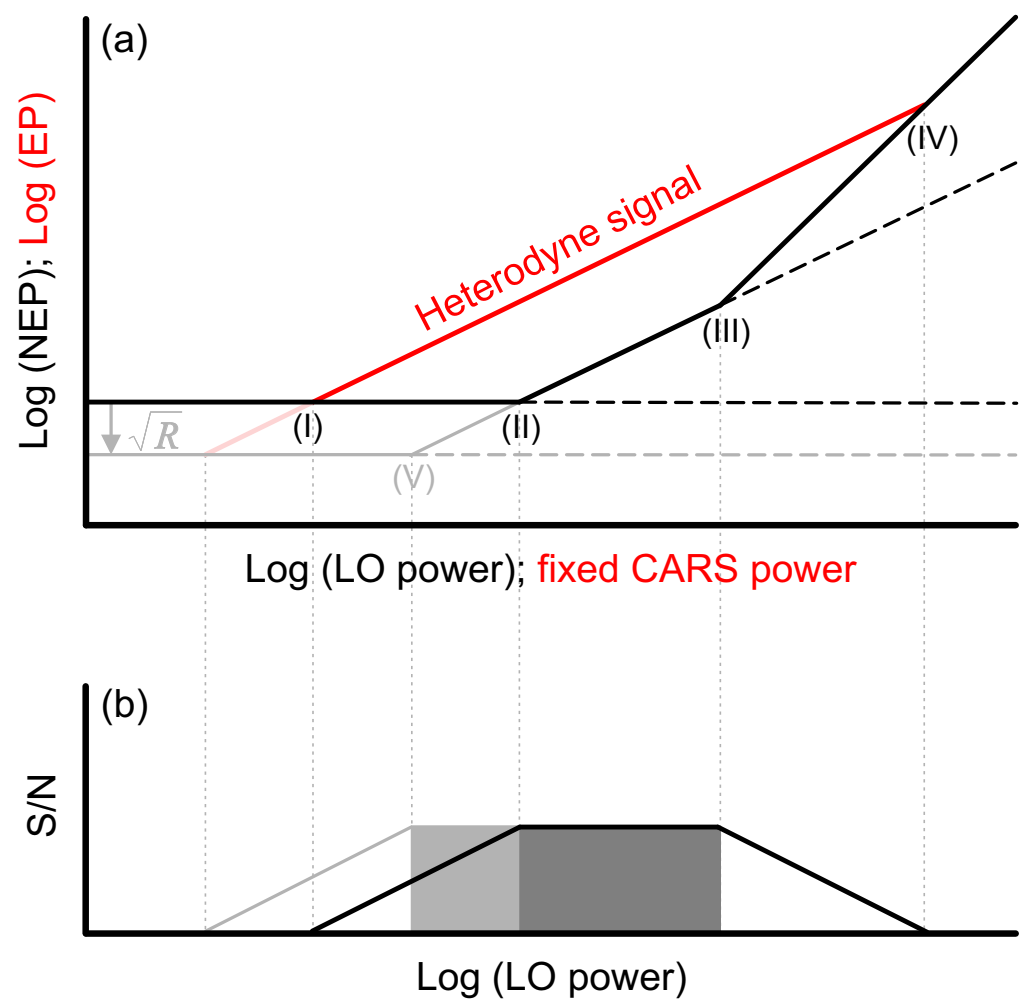

(3)

Figure 3.3: (a) The NEP of the detector as function of the local oscillator power, for a certain transimpedance amplification resistor $(R)$. Line 1: Detector dark noise; Line 2: Shot noise of local oscillator; Line 3: Relative intensity noise. Line 4: Detector dark noise decreased by $\sqrt{R}$ by using an $R$ time higher resistor $(R)$. The Heterodyne signal (in equivalent power $(E P))$ is shown as function of the local oscillator power at a fixed CARS power. (b) The signal to noise $(S / N)$, equivalent to the ratio between the noise and heterodyne signal.

\section{Resistor choice (R)}

The heterodyne CARS signal scales with the root of the local oscillator intensity, see equation 3.6 and figure 3.3. The moment the shot noise dominates the total noise (II), the maximum signal to noise ratio is reached. The signal-to-noise ratio decreases when the RIN sets in (III). The range of the local oscillator powers that yield the highest signal-tonoise ratio will be referred as the shot noise window.

The size of the shot noise window is determined by the choice of the resistor $(\mathrm{R})$. To increase the shot noise window a higher $\mathrm{R}$ can be cho- 


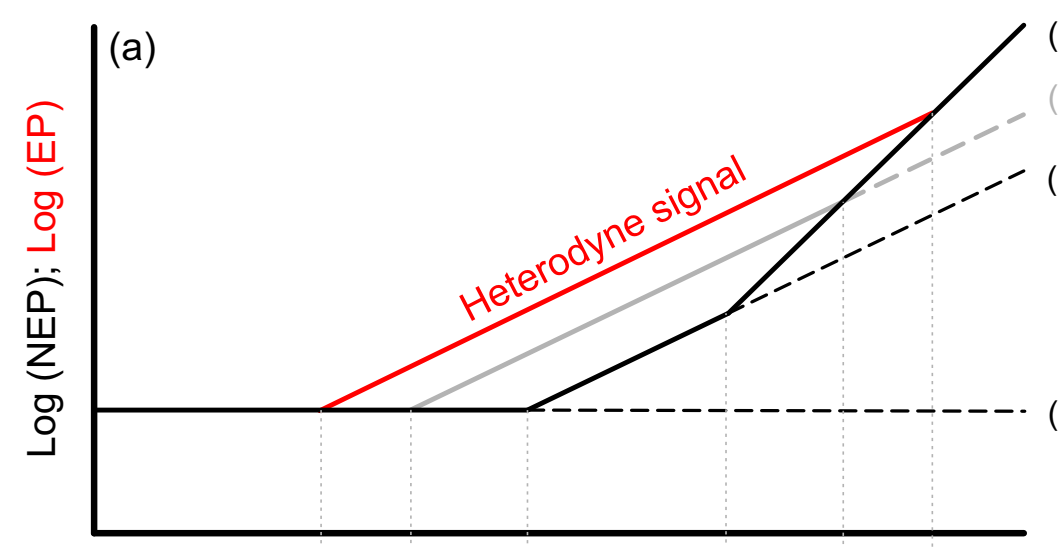

\section{Log (LO power); fixed CARS power}

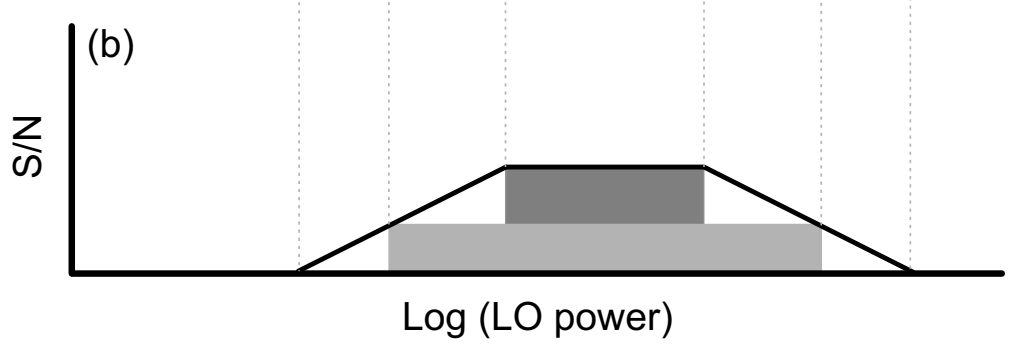

Figure 3.4: The role of Quantum Efficiency (QE) on the shot noise window. Line 2 represents the shot noise with a high QE. Line 4 represents the shot noise with a low QE, showing a broadening of the window for shot noise limited detection. However, the lower $Q E$ reduces the signal linearly and therefor the $S / N$ is also reduced to a lower level (light gray in part (b)).

sen, resulting in a lower equivalent dark noise - NEP power scales with the root of $\mathrm{R}$ - and extending the shot noise window (V). However, a higher $\mathrm{R}$ decreases the amplifier bandwidth. If the bandwidth is not large enough, a lower resistor can be used to increase the detection bandwidth. See appendix A.1 for a calculation example of how the NEP depends on the resistor.

As long as the amount of local oscillator is chosen such that the detection is shot noise limited, the signal to noise ratio is maximized and is independent of the choice of resistor. 


\section{Quantum efficiency (QE)}

The QE represents the number of photons converted to electrons by the detector. Typical QE's at $800 \mathrm{~nm}$ are up to $70 \%$ for a photodiode and $0.1-10 \%$ for a PMT . The QE is related to the detector sensitivity as

$$
\text { detector sensitivity }[A / W]=\frac{Q E \cdot e}{E},
$$

where $e$ is the electron charge and $E$ is the photon energy of the detected light. The level of the detected shot noise in the detector current (number of electrons) compared to the optical shot noise (number of photons) scales inversely with the root of the QE (equation 3.11). A lower QE therefore shifts line 2 in figure 3.4 upwards. The position of the RIN (line 3 ) is unaffected as it scales directly with the number of photons. This seems to improve the detection window by allowing higher levels of local oscillator but in reality deteriorates the detection sensitivity, see figure 3.4. The (shot) noise level as function of the $\mathrm{QE}$ of the detector is given by

$$
N E P_{\text {Shot noise at } Q E}=\frac{N E P_{\text {Shot noise at } Q E=1}}{\sqrt{Q E}} .
$$

The higher the QE of the detector, the smaller the shot noise window, but the better the signal to noise ratio.

\section{Experimental}

Figure 3.5 depicts the phase-preserving nonlinear chain used in the experiment. It starts with a passively modelocked $\mathrm{Nd}: \mathrm{YVO}_{4}$ laser (Spectra Physics Vanguard or Coherent Paladin) generating $~ 15$-ps pulses at a repetition rate close to $80 \mathrm{Mhz}$. The output at $1064 \mathrm{~nm}$ is partially frequency doubled to pulses of $\sim 12$ ps at $532 \mathrm{~nm}$. The $532 \mathrm{~nm}$ light synchronously pumps a homebuilt $[62,57]$ or commercial (APE Levante Emerald) optical parametric oscillator (OPO) generating pulses of $\sim 6.5$ ps at the signal and idler wavelength. For detection, a Centronic BPX65 (1*1 mm sensitive surface) photodiode and a Hamamatsu R1463 PMT are employed in combination with a transimpedance amplifier based on a Burr Brown OPA655P OPAMP.

For the following measurement the OPO idler is tuned to $1578 \mathrm{~nm}$ so that the difference frequency between the fundamental and idler matches the $\mathrm{C}-\mathrm{H}$ stretch vibration at $3060 \mathrm{~cm}^{-1}$ in toluene. The OPO signal (and 


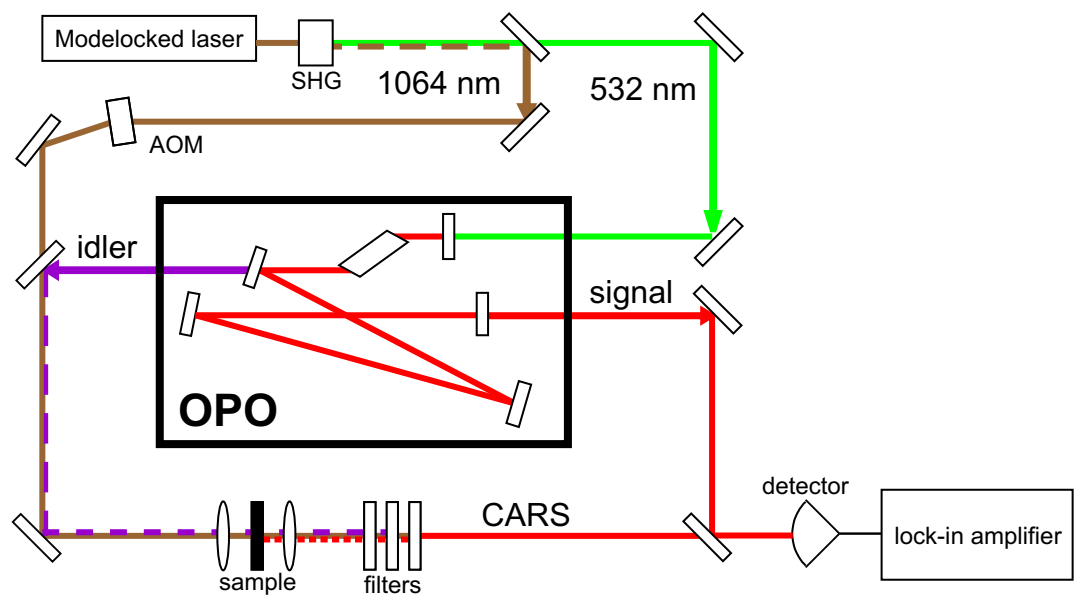

Figure 3.5: Setup of the optical chain for the phase-preserved generation of wavelengths for the CARS process.

CARS signal) wavelength is at $802.7 \mathrm{~nm}$. The toluene sample is held between two cover glasses and is approximately $15 \mu \mathrm{m}$ thick. Tens of $\mathrm{mW}$ of fundamental and idler are focused using a $0.60 \mathrm{NA}$ air objective. The CARS signal is collected with a 0.65 NA air objective. Filters subsequently remove the idler and fundamental.

To obtain heterodyne interferometric detection the CARS frequency is phase shifted by an acousto-optical modulator (AOM), Isomet $1205 \mathrm{C}$ 2 , in the $1064 \mathrm{~nm}$ branch. The AOM is placed in the $1064 \mathrm{~nm}$ beam, rather than the signal or idler beam, because this wavelength does not change as the OPO is tuned for a particular vibrational frequency. The $\mathrm{AOM}$ is driven at $80.00 \mathrm{MHz}$, effectively shifting the $1064 \mathrm{~nm}$ pulses, at a repetition rate of $79.98 \mathrm{MHz}$ (depending slightly on the room temperature), by $20 \mathrm{kHz}$. This modulation shifts the detection to a spectrally less noisy region, avoiding $1 / f$ noise. The $20 \mathrm{kHz}$ shift is translated to a shift of $40 \mathrm{kHz}$ at the CARS wavelength due to the involvement of two photons at $1064 \mathrm{~nm}$ in the CARS creation process. The CARS wavelength is combined on a detector with the OPO signal wavelength and the detected intensity is fed to a lock-in amplifier set to detect at $40 \mathrm{kHz}$. We integrate for $100 \mathrm{~ms}$ using a second order cut-off filter (BW $=1.58 \mathrm{~Hz}$ ).

Later for the imaging, the heterodyne detection was improved by detecting the laser repetition rate and using a phase locked loop with a 
voltage controlled oscillator (VCO). An external frequency of $50 \mathrm{kHz}$ is added to the detected laser repetition rate and applied to the AOM. The modulation frequency is fixed $(50 \mathrm{kHz})$ and not dependent anymore of the temperature of the laser, and therefor the cavity length. The detected intensity on the detector is fed to a lock-in amplifier set to detect at the $100 \mathrm{kHz}$, the maximum for our lock-in amplifier (Stanford Research SR530). Higher modulation frequencies can be used with a different lock-in amplifier and lower gain transimpedance amplifier.

Figure 3.6 shows the detected noise levels for a photodiode for three different transimpedances and for a PMT as a function of the local oscillator power. The noise levels are back calculated to noise equivalent input power (at $803 \mathrm{~nm}, 1.58 \mathrm{~Hz}$ bandwidth) for comparison between the detectors and different resistors. When the amount of local oscillator power on the detector is increased, the noise (signal without any CARS signal on the detector) first shows the dark count noise level (flat) followed by a section showing the shot noise of the local oscillator. This shows up as a slope of $1 / 2$ in a log-log plot of the noise versus the local oscillator power (parallel to line (I)). At some point the noise becomes dominated by the RIN of the local oscillator, scaling with a slope of 1 (parallel to line (II)). Since this contribution to the noise increases faster than the heterodyne signal (scales parallel to line (I)), the signal-to-noise ratio deteriorates. The section in which the noise follows line (I) is the window in which CARS signals can be detected while limited only by the shot noise. The shot noise line for the PMT lies substantially above that of the photodiode due to the low quantum efficiency (0.3\%). The PMT shows a large window for shot noise limited detection. The photodiode shows a window only when terminated by $1 \mathrm{M} \Omega$ or $10 \mathrm{M} \Omega$. At lower termination resistance the required local oscillator level introduces noise dominated by the RIN. Above $10 \mathrm{M} \Omega$ the OPAMP is no longer able to follow the $40 \mathrm{KHz}$ oscillations.

\subsubsection{Relative intensity noise (RIN)}

The relative intensity noise (RIN), describes the instability in the power level of a laser source and the RIN can be generated from cavity vibration, fluctuations in the laser gain medium or simply from transferred intensity noise from a pump source. When the RIN is not limited by the shot noise, the level of the RIN in the local oscillator can be reduced (for shot noise limited detection) by 


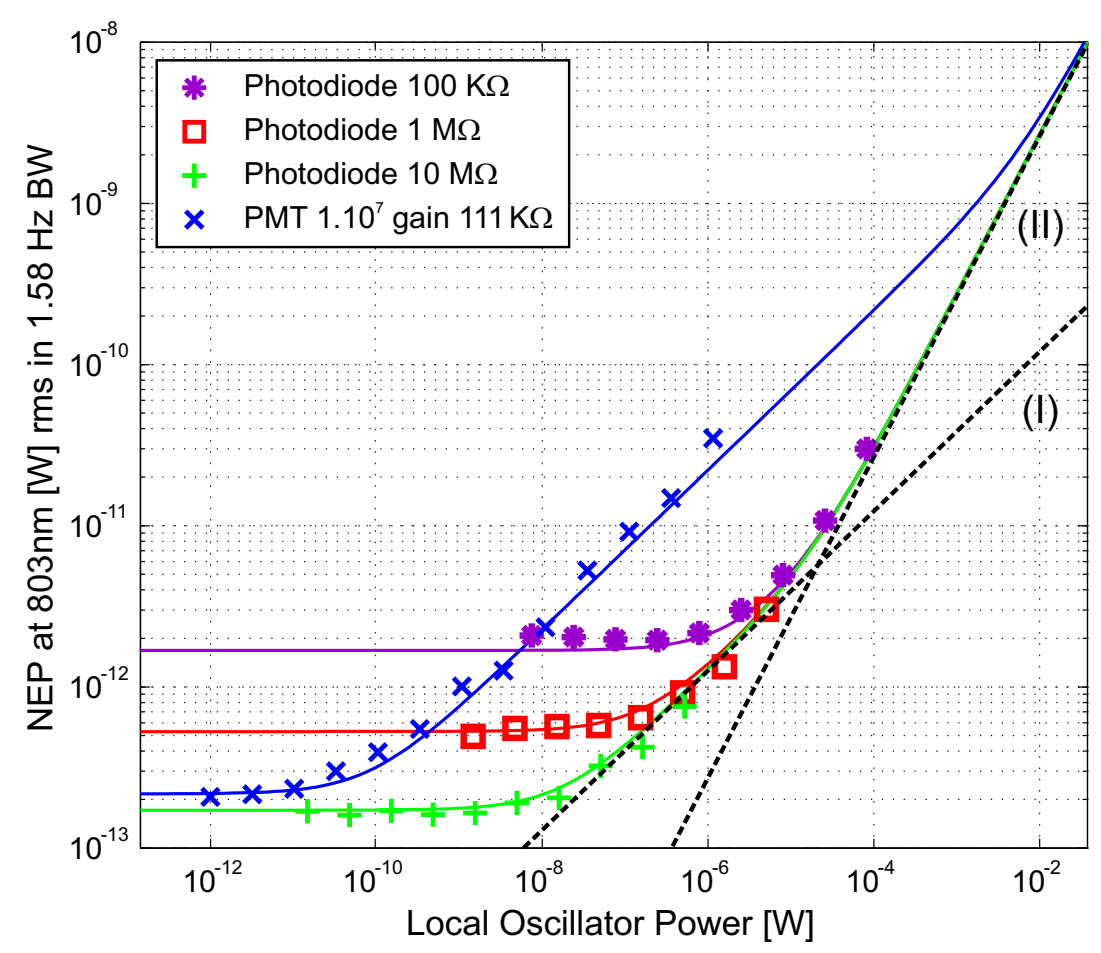

Figure 3.6: Noise as a function of local oscillator power. The noise is expressed in Noise Equivalent Power (NEP), Root Mean Square (rms) in $1.58 \mathrm{~Hz}$ bandwidth so that all lines can be compared directly. The dashed lines (I) and (II) have a slope of $1 / 2$ and 1 respectively. Continuous lines are simulations, symbols represent measured data.

- higher saturation of the OPO,

- higher modulation frequencies,

- and/or dual balance detection.

In particular the saturation level of the OPO is related to the RIN. If the OPO is not strongly saturated the RIN can dominate at (local oscillator) levels where the detector noise is not yet dominated by the shot noise, effectively closing the window for shot noise limited detection. The dependence of the RIN level on the saturation of the OPO is demonstrated in figure 3.7 where we plot both the OPO (signal) output power and the detected noise at the heterodyne frequency for a fixed level of local oscillator on the detector $(2 \mu \mathrm{W})$. Pumping the OPO with the Coherent Paladin (up to $12 \mathrm{~W}$ at $532 \mathrm{~nm}$ ) instead of our Spectra 


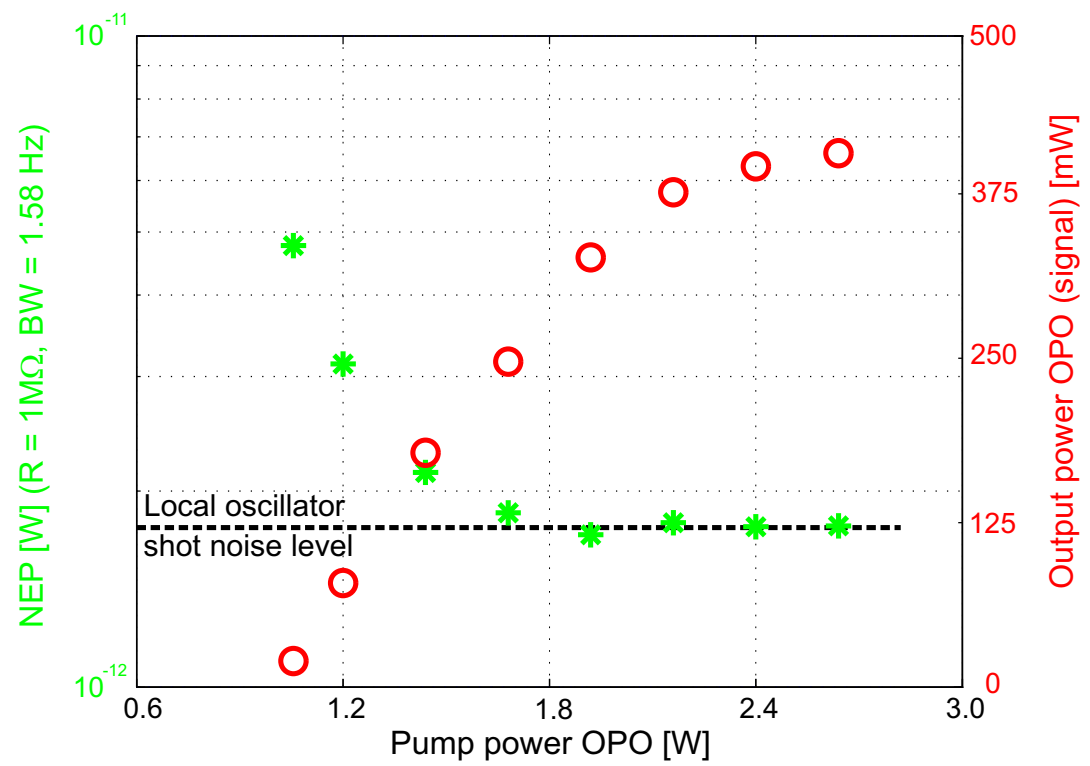

Figure 3.7: Noise (NEP, $R=1 M \Omega, B W=1.58 \mathrm{~Hz}$ ) for $2 \mu \mathrm{W}$ of local oscillator signal and the total signal output versus OPO pump power (532 $\mathrm{nm}$ inside the crystal)

Physics Vanguard (2 W at $532 \mathrm{~nm}$ ) increases saturation. The decrease in the RIN enables shot noise limited detection for lower transimpedance (and corresponding higher frequency) until the dark current noise of the detector dominates Johnson noise (R below $50 \mathrm{k} \Omega$ ).

In figure 3.8 the performance of the Levante Emerald OPO pumped by the Coherent Paladin laser is shown. This OPO is designed to operate at much higher pump (and output) levels than the home built OPO used for the characterization of the detector noise in figure 3.6. By pumping the OPO with the Paladin laser we can saturate the Levante Emerald to the point that even at lower transimpedance there is a window for shot noise limited detection. The lower transimpedance implies the possibility of detection at higher modulation frequencies and therefor higher scanning speeds. With $6.2 \mathrm{~W}$ of pump power, $1.9 \mathrm{~W}$ of signal and 1.0 $\mathrm{W}$ of idler power is available for the generation of CARS, and a $100 \mathrm{k} \Omega$ resistor can be used for shot noise limited detection with a bandwidth of $10 \mathrm{MHz}$.

Because the RIN is typically independent of laser power and falls off with frequency, higher modulation frequencies of the local oscilla- 


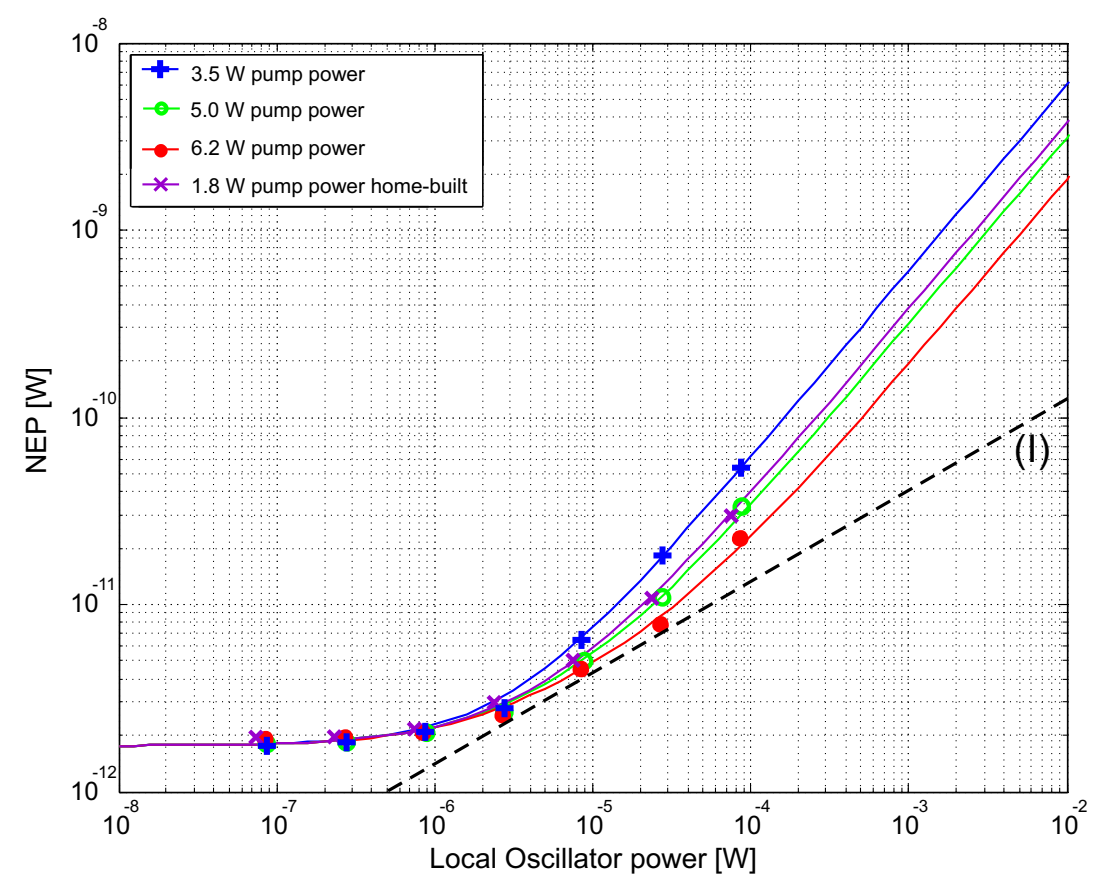

Figure 3.8: Noise ( $N E P, R=100 \mathrm{k} \Omega, B W=1.58 \mathrm{~Hz}$ ) for different pump powers (as measured outside the OPO). Combination of Coherent Paladin pump laser and APE Levante Emerald OPO. The dashed line shows the RIN for the homebuilt OPO. (I) Represents the shot noise level.

tor can lower the RIN level. With a photodiode the RIN spectrum is recorded, see figure 3.9. The laser RIN spectrum shows a broad noise band between 20 and $40 \mathrm{kHz}$ and a pronounced peak around $100 \mathrm{kHz}$. This noise is due to the relaxation oscillations of the laser source [63]. The OPO RIN spectrum amplifies the spectrum of the laser source and shows that the OPO is the dominant RIN level source. Saturation of the OPO, as shown in figure 3.9(b), reduces the RIN level significantly, but the modulation frequency of the local oscillator should be higher than $50 \mathrm{kHz}$ to overcome high RIN levels.

To suppress the RIN even more, a dual balance detection setup can be used [64]. A dual balance detection setup is commonly used in Optical Coherence Tomography (OCT) [65] to suppress the RIN level and obtain shot noise limited detection. The modulated local oscillator and signal are mixed in a 50/50 beamsplitter. Both branches are detected and subtracted from each other. This results in the DC offset terms 

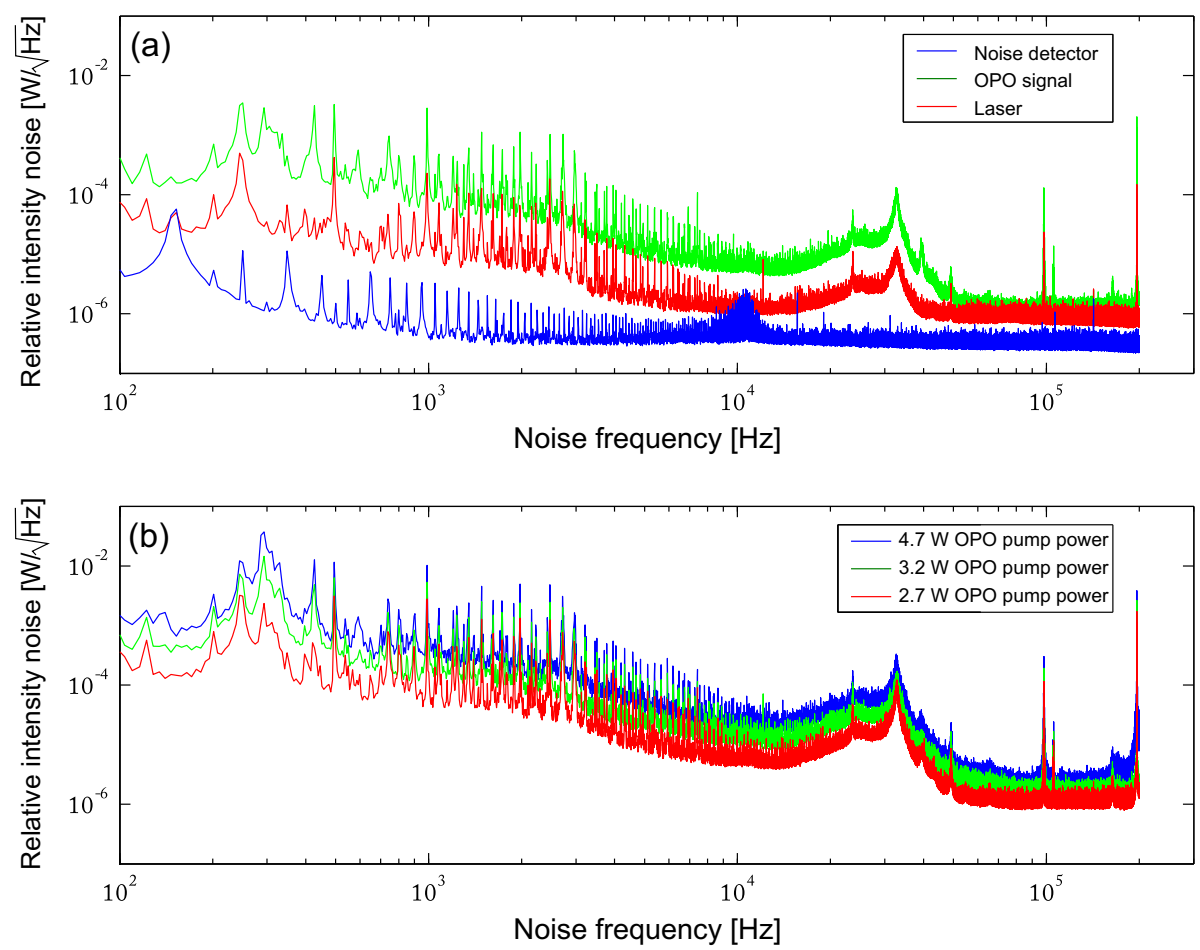

Figure 3.9: The relative intensity noise (RIN) spectrum. (a) RIN spectrum of laser, OPO and noise spectrum of detector. (b) RIN spectrum of the signal wavelength of the OPO (local oscillator) at different OPO pumping powers.

canceling out and the modulation signal being twice as high, due to the phase shift of 180 degrees in one of the branches. The dual balance detection scheme is not used because the local oscillator is already shot noise limited for the current modulation frequency and detection bandwidth. When higher bandwidths are required for real time imaging, a dual balance detection setup is required.

\subsection{Detection improvement}

Heterodyne detection gives the opportunity to improve the detection sensitivity. Figure 3.10 shows the measured heterodyne CARS signals for a fixed local oscillator level ( $50 \mathrm{nW}$ for the photodiode and $2 \mathrm{nW}$ for the PMT), where the intensity of the CARS signal is varied by introdu- 


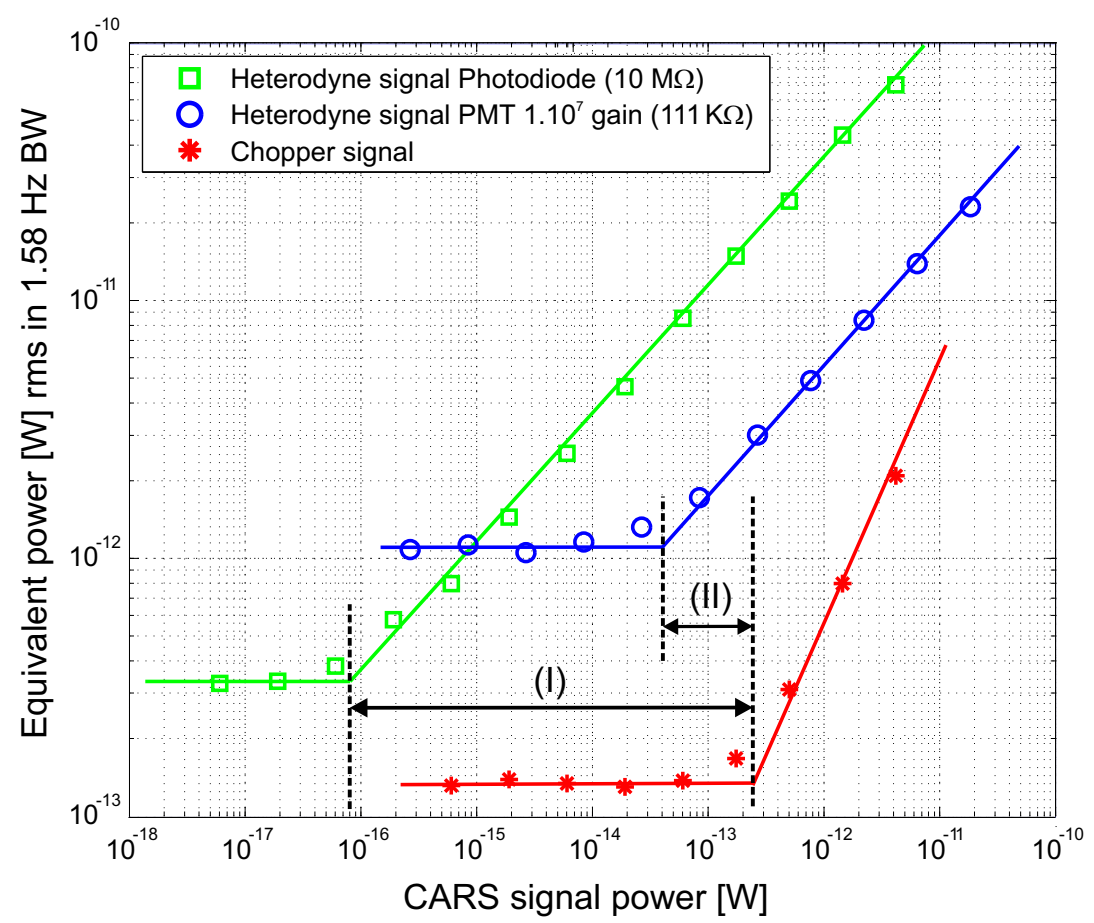

Figure 3.10: Heterodyne signal and direct detected signal (rms in 1.58 $\mathrm{Hz} B W$ ) versus CARS power. (I) represents the improvement factor of 3500 in detection sensitivity between the heterodyne detection and direct (chopper) detection signal for a photodiode. (II) represents the improvement factor of 7.5 for detection with a PMT.

cing attenuation filters in that part of the beam. For comparison we also show the direct detection of the CARS signal by removing the local oscillator and chopping the CARS beam with a mechanical chopper. The chopped signal shows the expected direct dependence on the CARS level with a slope of 1 . The heterodyne signal scales only with the root of the CARS signal and therefor decreases with a slope of $1 / 2$, see equation 3.6. For increasing attenuation of the CARS signal, the direct detection signal disappears in the detector noise whereas the heterodyne detection continues to lower levels before disappearing into the shot noise of the local oscillator. For detection by a photodiode that is terminated with $10 \mathrm{M} \Omega$, the minimum CARS signal that can be detected using the heterodyne detection is a factor 3500 lower in comparison to what can be detected directly. Since the amount of local oscillator that we apply is such that the noise is limited by the shot noise (see figure 3.6) we detect 


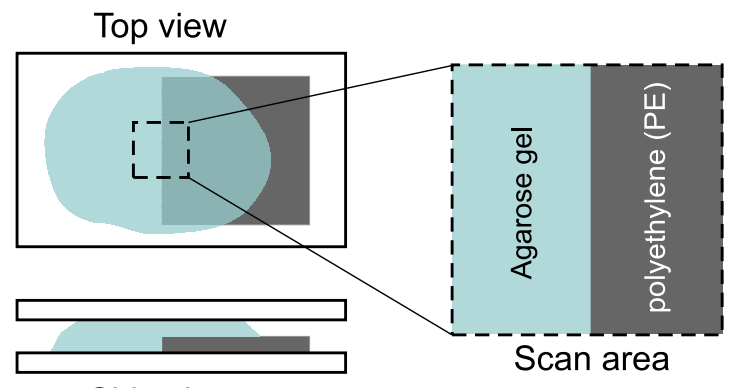

Side view

Figure 3.11: Schematic of sample used for spectroscopic measurement of amplitude and phase.

the CARS signal with a precision limited only by the shot noise. Detection of lower absolute signal levels is possible for longer integration times.

The PMT has a very low noise level and high gain so that the improvement by heterodyning is not so impressive (only a of factor 7.5). However, the quantum efficiency of the PMT is so low that the minimum detectable signal is considerably higher than for the heterodyne detection using a photodiode. There are PMTs with better QE commercially available (QE up to $15 \%$ are quoted). For those PMTs the heterodyne detection minimum would approach the limit for the photodiode.

\subsection{Phase detection}

As shown in the previous section the detection sensitivity can be significantly improved with heterodyne CARS. Another advantage of heterodyne CARS is the ability to detect the phase of the molecular response. The power of the signal (local oscillator) of the OPO is attenuated to a level within the window for shot noise limited detection (few $\mathrm{nW}$ ) on the PMT (Hamamatsu R3896) and is combined with the fundamental $(1064 \mathrm{~nm})$ and the idler, respectively $\omega_{\text {pump }}$ and $\omega_{\text {Stokes }}$ (both tens of $\mathrm{mW}$ ). At this low power level, the signal of the OPO does not contribute to the CARS process between fundamental and idler. The local oscillator can thus be sent collinear with the other beams through a sample without disturbing the CARS process, avoiding path length differences in scanning microscopy. The three beams are scanned over the sample by galvano mirrors (Olympus FluoView 300, IX71) and focused by a 1.2 NA $60 \mathrm{x}$ water objective (C.A.R.S., Olympus) lens into the sample. 

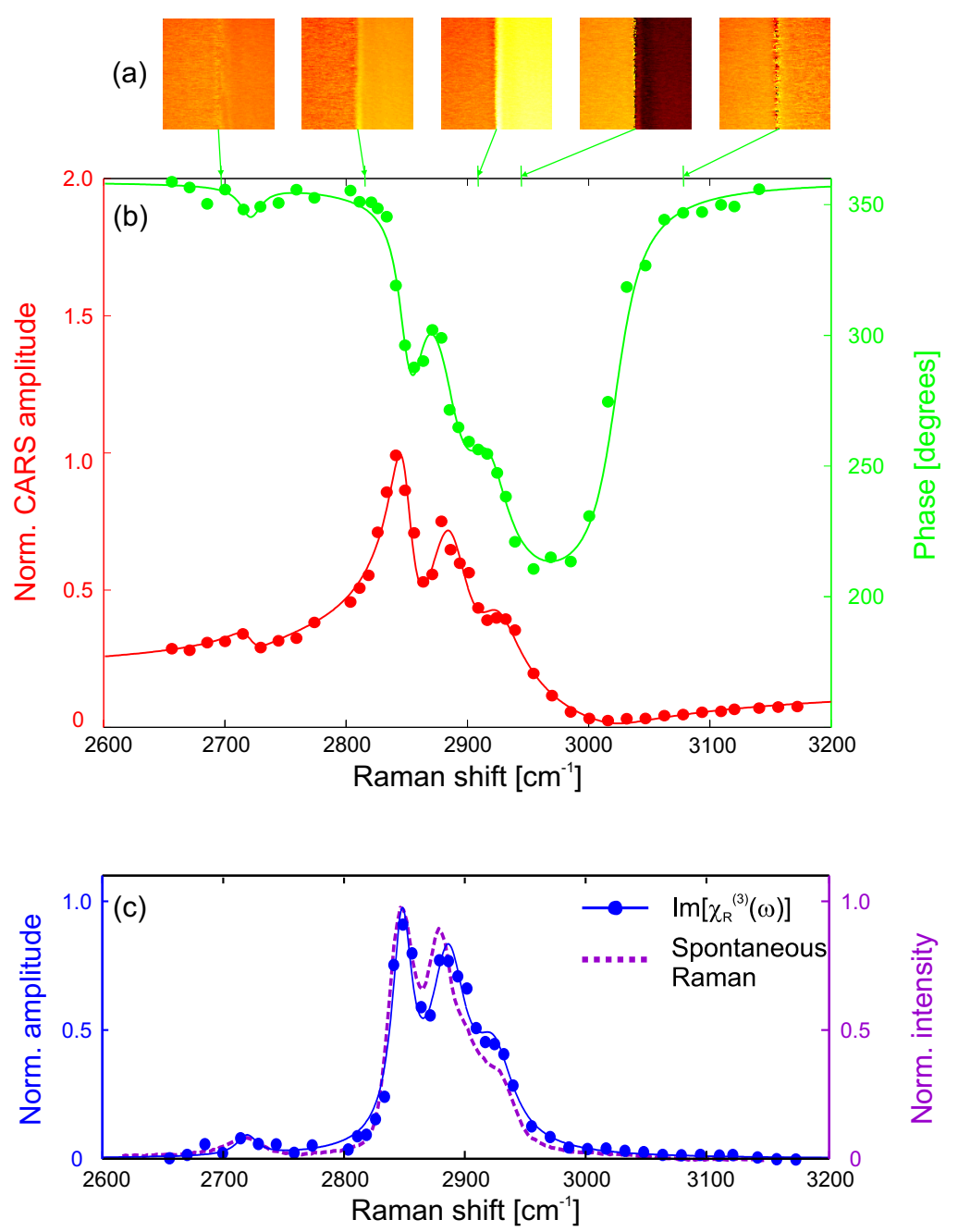

Figure 3.12: (a) The CARS amplitude spectrum of PE (red) and the associated phase of the spectrum (green). The symbols are data and the solid curves are fits based on multiple Lorentzian bands as in Eq. 2.3. (b) The magenta dotted line is the spontaneous Raman spectrum of PE and in blue the imaginary part of the data in panel (c) is shown.

The generated CARS signal and the local oscillator are collected by a collimation lens. The fundamental and idler are filtered by two bandpass filters (Chroma 825-150-2p) and the signal is detected by the PMT.

To demonstrate the phase detection capabilities a spectroscopic measurement of the phase over several overlapping vibrational resonances is 
presented. The lock-in amplifier is set to $1 \mathrm{~ms}$ integration time and a scanning speed of 5000 pixels/sec over $256 \times 100$ pixels is used. From the lock-in amplifier both the amplitude and phase for every pixel are obtained. The sample, shown schematically in figure 3.11, is comprised of two adjacent areas. One side consists of agarose gel, which contributes mainly to the non-resonant signal. The other side contains polyethylene $(\mathrm{PE})$, which gives mostly resonant signal. Scanning the sample reveals a phase step between the non-resonant and resonant sides of the sample. The height of the phase step depends on the spectral position with respect to the vibrational resonance of PE. Due to the small scan area, there is no phase difference caused by the curvature of the field of view, as was verified by measurements on a sample containing only resonant material. The measured amplitude and phase are shown in figure 3.12(b). While tuning the OPO through the high frequency vibrational spectrum of $\mathrm{PE}$, several measurements are recorded and averaged for each region in the sample, see figure 3.12(a). The averages are corrected for intensity variations of the input wavelengths. The phase is determined as the difference in phase between the non-resonant and the resonant side of the image.

To show that the phase data is in agreement with the amplitude data, the complex dataset, containing both the phase and amplitude information, is fitted to four vibrational levels of PE, modeled as complex Lorentzians bands (equation 2.3), with a real offset for the non-resonant background. The fit results in the solid curves for the amplitude and phase as shown in figure 3.12(b), indicating that the measured phase and the spectral amplitude are directly related, as predicted. The phase has an additional offset of 8 degrees due to the refractive index difference between $\mathrm{PE}$ and agarose gel. The phase of the molecules is the difference between the driving field, at the difference frequency between pump and Stokes, and the motion of the dipoles in the sample. When the driving frequency is low (left) the response is dominated by the nonresonant response, which has a phase of zero. As the first resonance at $\sim 2700 \mathrm{~cm}^{-1}$ ) is approached, the phase of the resonant response rotates, but since this response is weak the phase does not rise to a full phase difference of $\pi$. Subsequent stronger resonances pull the phase further down. At frequencies above the resonances the non-resonant response starts to dominate again, returning the phase difference to zero.

A direct comparison with the spontaneous Raman spectral intensity of $\mathrm{PE}$ can now be made. Figure 3.12(c) shows the comparison. The solid blue line shows the imaginary part of the complex CARS dataset and 
the purple dotted line shows the spontaneous Raman spectrum. The very good agreement between the fit and the Raman data indicates that the setup is capable of accurate phase detection by heterodyne CARS.

\subsection{Background rejection}

From equation 3.5 it is seen that heterodyne CARS enables to distinguish between the resonant and non-resonant CARS signals. In this section heterodyne CARS detection for background free imaging will be demonstrated. A sample consisting of lipids suspended in agarose gel is used. Figure 3.13 shows the CARS intensity signal generated by the idler and fundamental. The images consist of $152 \times 152$ pixels representing $35 \times 35$ $\mu \mathrm{m}$. Figure 3.13(a) is scanned at 5000 pixels/sec and measured directly with the PMT, 3.13(b,e) are scanned at 600 pixels/sec and detected with the photodiode and show the signal after the lock-in amplifier, with an integration time of $3 \mathrm{~ms} /$ pixel. A cross section (3.13(c)) is given at a position (indicated by the green line) where the lipid can hardly be seen in figure 3.13(a). Heterodyne CARS images (figure 3.13(b,e)) show the amplitude and the phase of the image respectively. The amplitude image shows several large lipid regions and some smaller ones. The cross section (figure $3.13(\mathrm{~d}, \mathrm{~g})$ ) intersects 4 of the lipid regions, as numbered under panel 3.13(b). The smallest lipid region (I) has a signal-to-background ratio of only 1.5 due to the strong non-resonant signal of the agarose gel. Analyzing the phase image shows that this first region causes a clear phase step compared to the non-resonant signal, which has a flat phase profile across the image. By assigning the phase in between the lipid regions (yellow in figure 3.13(e)) to the non-resonant phase, an absolute phase is obtained between the local oscillator and the CARS signal and the resonant part can be extracted. The corresponding image is shown in figure 3.13(f). The first lipid region is now clearly revealed and the signal-to-noise ratio has been improved by more than one order of magnitude $(>15)$.

\subsection{HIP-CARS}

For recording background free spectra of solutions, we demonstrate a relatively easy-to-use method that exploits the synergies between heterodyne amplification (section 3.4) and the polarization sensitivity (section 2.4) of the CARS response. This heterodyne interferometric polariza- 

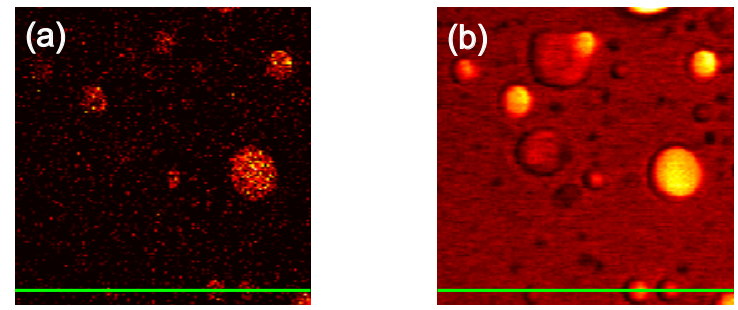

(I) (II) (III) (IV)
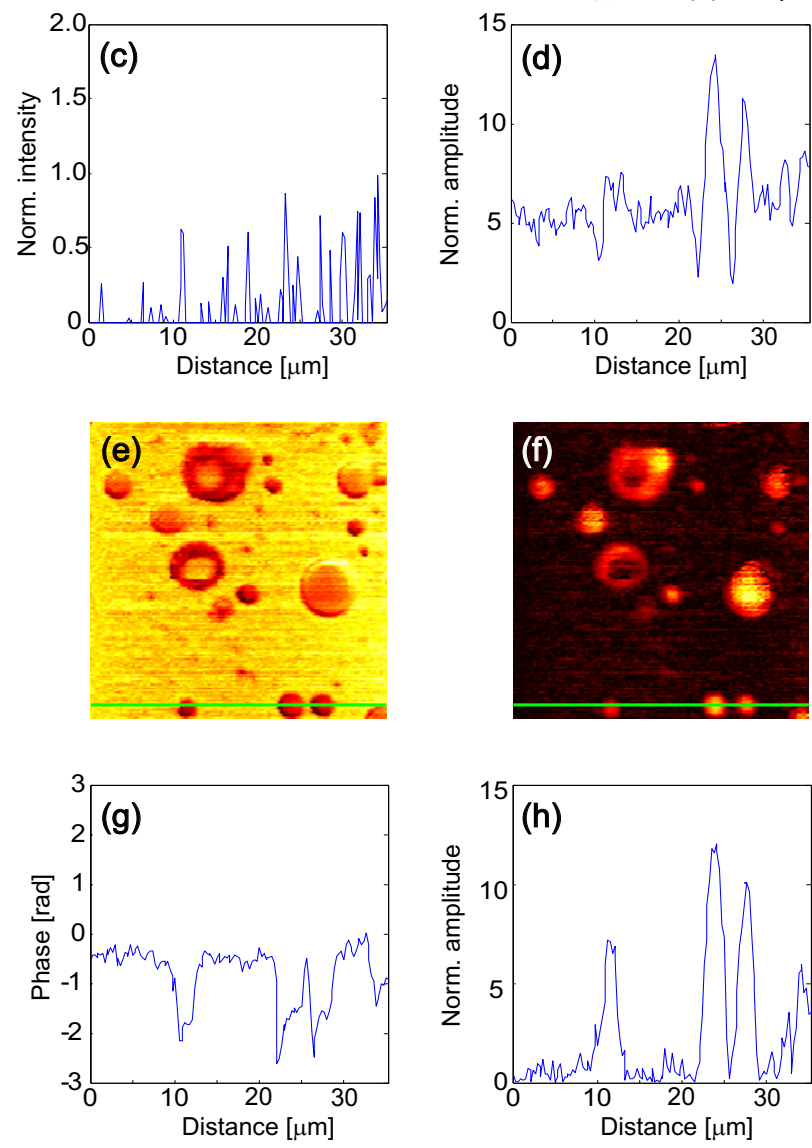

Figure 3.13: The sample contains lipid suspended in agarose gel imaged at $2845 \mathrm{~cm}^{-1}$. The image size is $35 x 35 \mu \mathrm{m}$. (a) Forward intensity CARS image obtained with $1064 \mathrm{~nm}$ (pump and probe) and idler (Stokes). (b) and (e) are respectively the amplitude and phase image obtained with heterodyne CARS. (f) Im $\chi_{R}^{(3)}$, the non-resonant free image of the sample. (c,d,g and $h$ ) Cross sections of the images above at the thin green line at the bottom. 
tion CARS (HIP-CARS) is ideally suited for turnkey CARS systems, since it requires minimal realignment and is capable of addressing vibrational resonances in the biologically-important fingerprint region (600$1650 \mathrm{~cm}^{-1}$ ) without any interference of the non-resonant background. The main drawback of PCARS, the loss of a significant portion of the resonant signal, is overcome by the interferometric amplification. The major drawback of heterodyne CARS is that a reference phase (previously taken as the phase of the non-resonant background) is required to separate the resonant and non-resonant parts. Since this separation is achieved by the polarization selection in HIP-CARS, there is no need to detect an absolute phase. On the practical side, the heterodyne amplification allows for detection on a photodiode rather than a photomultiplier tube, normally used in PCARS detection. The photodiode sensitivity extends to longer wavelengths so that lower wavelength IR inputs can be used, which gives access to lower frequency vibrational resonances.

The microscope is replaced by two objective lenses. The pump laser is a Coherent Paladin modelocked laser and $530 \mathrm{~mW}$ of green laser power is used to synchronously pump a specially designed home-built picosecond PPLN-based OPO to address vibrational resonances in the fingerprint region. More details about the OPO can be found in [59]. The idler and signal beams generated from this OPO are used as the Stokes in the CARS process and local oscillator, respectively, while the remainder of the laser fundamental at $1064 \mathrm{~nm}$ is used for both the pump and probe waves. The fundamental is phase modulated by an AOM locked to a frequency $50 \mathrm{kHz}$ higher than the laser repetition rate. Half-wave and quarter-wave plates in all three beams allow for precise control of all polarizations within $2^{\circ}$ and compensate for birefringence induced by the dichroic mirrors. The local oscillator power is attenuated to about $10 \mathrm{nW}$. The average pump/probe power at the sample is $130 \mathrm{~mW}$, while the Stokes power is about $30 \mathrm{~mW}$. The beams are focused into the sample with a Nachet 0.65 NA IR-corrected objective lens and collected with a long working distance Nikon 0.40 NA objective lens. The analyzer in this experiment is a polarizing beam cube with an extinction ratio of larger than $10^{4}: 1$. The detector is an InGaAs photodiode (ThorLabs SM05PD4B) followed by a $10 \mathrm{M} \Omega$ transimpedance amplifier. The amplitude of the modulation on the detected HIP-CARS signal is measured with a Stanford SR570 lock-in amplifier referenced at $100 \mathrm{kHz}$. The recorded CARS spectra are processed to account for transmission characteristics of the filters, spectral responses of the detector, and fluctuations in OPO power. 


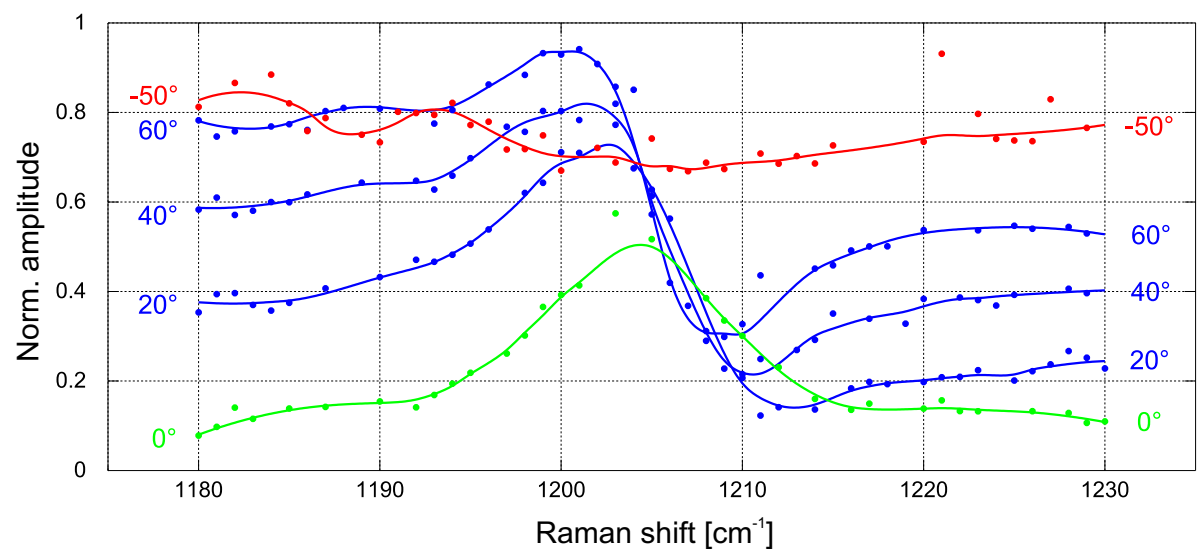

Figure 3.14: HIP-CARS amplitude of toluene around $1200 \mathrm{~cm}^{-1}$ at different angles of the analyzer. Angles are relative to the original PCARS position of the analyzer.

Rotation of the analyzer reveals the non-resonant and resonant contributions of the transmitted HIP-CARS signal and combinations thereof, as shown in figure 3.14. The HIP-CARS lineshapes appear symmetric around the peaks and do not contain the peak shift to the lower wavenumber side and characteristic dip in the signal on the higher wavenumber side that are characteristic of isolated CARS responses, see section 2.1.2. In practice, the non-resonant background is not completely removed. Birefringence in the sample lead to a slight depolarization of the CARS fields, and a small amount of the non-resonant component is interferometrically amplified. However, this amount is insignificant. A larger error is introduced by the small (several degrees) rotation of the polarization of the Stokes beam while tuning the OPO due to the dichoic mirrors. This alters the polarization of both the resonant and non-resonant beams, thus allowing more non-resonant background signal to pass the analyzer.

To demonstrate the background-free addressing of vibrational resonances in the fingerprint region, three spectra of toluene where recorded to compare HCARS, HIP-CARS and spontaneous Raman from 600-1650 $\mathrm{cm}^{-1}$, see figure 3.15. The HCARS and HIP-CARS scans are both taken in 5 measurements of about two minutes each, at the maximum scan rate of the OPO, limited by the heating/cooling rate of the nonlinear PPLN crystal. Comparing HIP-CARS with the Raman spectra, 


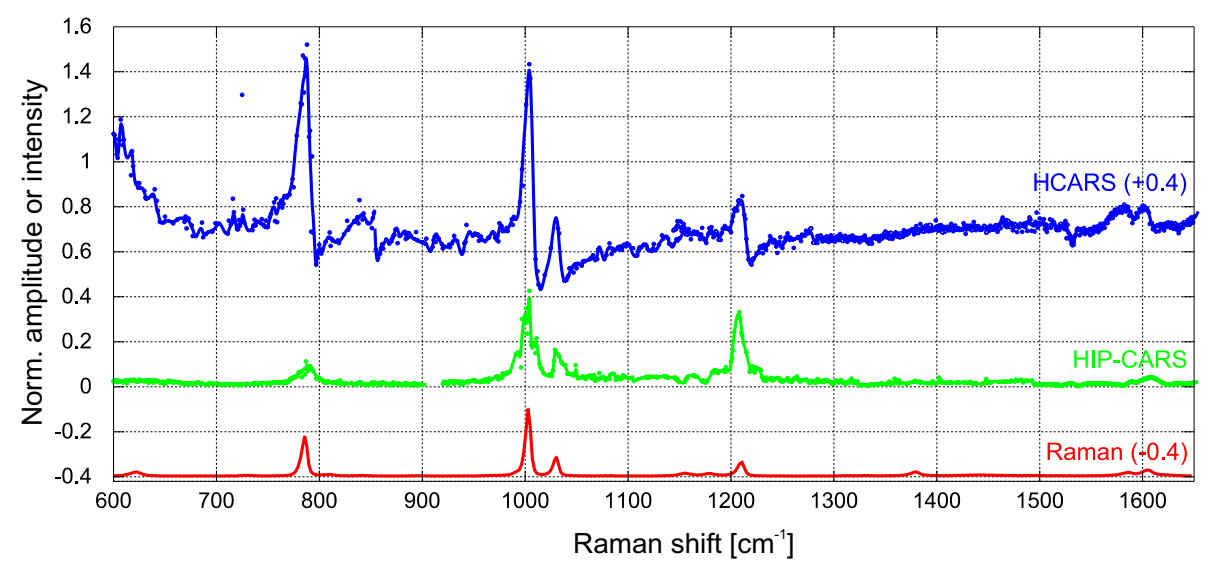

Figure 3.15: HIP-CARS spectrum of toluene (middle) in the fingerprint region. The top line shows the HCARS spectrum, offset by 0.4. The spontaneous Raman spectrum is offset by-0.4. Because the HCARS and $H I P-C A R S$ spectra are taken in consecutive measurements of approximately 200 wavenumbers each, small discontinuities appear in these spectra.

it can be concluded that they are in good agreement over the full fingerprint region. This demonstrates that HIP-CARS is an easy-to-use CARS technique to suppress the non-resonant background and obtain reliable vibrational information in solutions. 
Chapter 3 


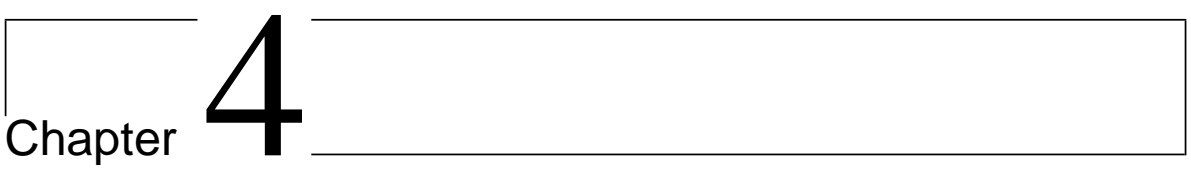

\section{Vibrational Phase Contrast CARS}

In mixtures of compounds the resonant CARS signal of less abundant constituents may be overwhelmed by the non-resonant background, preventing detection of resonant molecules. In this chapter a method is demonstrated to obtain the phase of the oscillators in the focal volume, which allows for discrimination between the resonant and non-resonant molecules. The phase is measured with respect to the local excitation fields using a cascaded phase-preserving chain. It is measured pointby-point and takes into account refractive index changes in the sample, phase curvature over the field-of-view and interferometric instabilities. The detection of the phase of the vibrational motion can be regarded as a vibrational extension of the linear (refractive index) phase contrast microscopy introduced by Zernike in 1933 [1]. 


\subsection{Introduction}

Heterodyne CARS achieves rejection of the non-resonant CARS signal, without rejection of resonant signal, by detection of the amplitude and phase of the CARS signal, see chapter 3 . This is achieved by mixing the CARS signal with a stable external reference signal. In this chapter we further extend the cascaded phase-preserving chain, by adding a scheme for the detection of the phase of the excitation field, created by the superposition of the pump and Stokes beam. Subtraction of the excitation phase from the heterodyne phase yields the phase of the vibration of the molecules with respect to the excitation fields, taking into account the changes in refractive index, the curvature of the field-of-view and any other phase disturbances in the sample. This clean phase provides a complete rejection of the non-resonant background and reveals the details to an arbitrary precision. Further, the technique offers shot noise limited performance and precision that is limited by integration time only.

The possibility of plotting the detected vibrational phase and amplitude in the complex plane with VPC-CARS, offers a new insight in CARS microscopy and opens up new possibilities. The complex plane allows for component analysis, dissolution monitoring and the measurement of reaction dynamics while addressing only a single vibrational mode. These new applications will be demonstrated in the second part of this chaper.

\subsection{Vibrational Phase Contrast CARS}

\subsubsection{Detection of the local excitation phase}

The phase difference between the total CARS field and the excitation field is $\phi_{\chi}$, see equation 3.2 and 3.5. This is the phase difference that distinguishes the real (non-resonant) and the imaginary (resonant, Raman) parts. However, this is not the heterodyne phase measured in the focal volume; even for co-propagating fields this phase is disturbed by refractive index changes in the sample, curvature of the field-of-view and interferometric instabilities. Detecting the local excitation phase and using this "base" phase to correct the heterodyne detected phase gives the accurate phase of the oscillators in the focal volume, hence forth referred to as "vibrational phase". For our cascaded chain (figure 


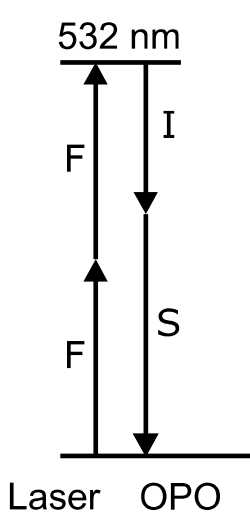

Laser OPO

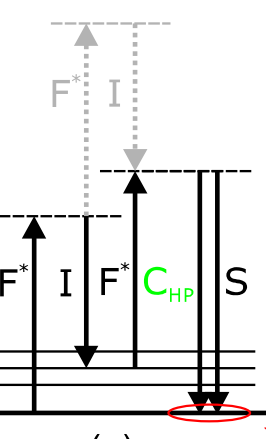

(a)

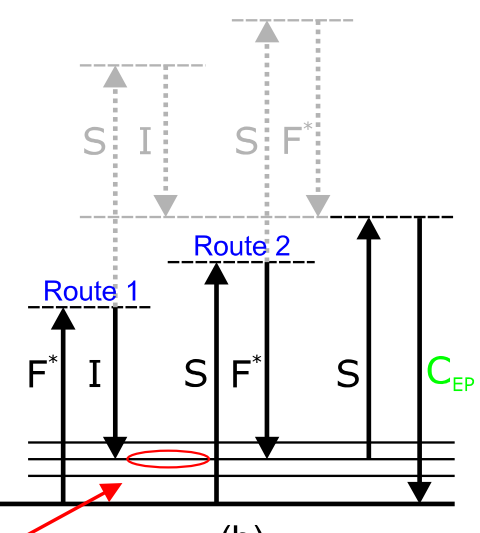

(b)

Figure 4.1: Schematic of the cascaded phase-preserving chain between laser, OPO and the CARS process. (a) The heterodyne phase detection scheme. (b) The local excitation phase detection scheme. $F=$ laser fundamental $(1064 \mathrm{~nm}), F^{*}=$ modulated laser fundamental, $I=O P O$ idler, $S=O P O$ signal and $C=C A R S$ signal. Given in black and gray are the resonant and non-resonant CARS processes, respectively.

4.1(a)), the phase difference $\left(\Delta_{1}\right)$, obtained by the interference between the local oscillator (the OPO signal beam) and the CARS signal $\left(C_{H P}\right)$ is given by:

$$
\begin{aligned}
\Delta_{1} & =\phi_{S}-\phi_{C_{H P}} \\
& =\phi_{S}-\left(2 \phi_{F^{*}}-\phi_{I}+\phi_{\chi}\right) \\
& =2 \phi_{F}-2 \phi_{F^{*}}-\phi_{\chi}
\end{aligned}
$$

where $F^{*}$ is a frequency modulated version of $F$. Figure 4.1(b) shows the detection of the local excitation phase, where there are two separate routes to the same vibrational level (or continuum level) creating an interference that can be probed by the OPO signal and detected at the $C_{E P}$ wavelength, given by $2 S-F^{*}$. The interference arises from the phase difference between the two pathways $\left(\Delta_{2}\right)$ :

$$
\begin{aligned}
\Delta_{2} & =\phi_{\text {Route } 2}-\phi_{\text {Route } 1} \\
& =\left(\phi_{S}-\phi_{F^{*}}\right)-\left(\phi_{F^{*}}-\phi_{I}\right) \\
& =2 \phi_{F}-2 \phi_{F^{*}} .
\end{aligned}
$$




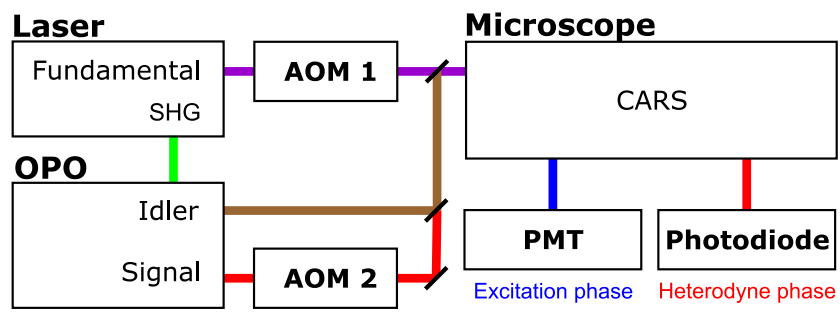

Figure 4.2: Schematic of the setup, where AOM 1 is phase modulated and $A O M 2$ is amplitude modulated

The difference between the $\Delta_{2}$ and $\Delta_{1}$ is now purely due to the interaction with the vibrational phase response $\phi_{\chi}$.

$$
\begin{aligned}
\text { Vibrational phase } & =\Delta_{2}-\Delta_{1} \\
& =\left(2 \phi_{F}-2 \phi_{F^{*}}\right)-\left(2 \phi_{F}-2 \phi_{F^{*}}-\phi_{\chi}\right) \\
& =\phi_{\chi} .
\end{aligned}
$$

Phase disturbances will occur within the acquisition time of one frame and over the full field-of-view. Using alternating phase detection on a point-by-point basis over the sample overcomes these interferometric instabilities.

\subsubsection{Detection setup}

The key elements of the setup (see figure 4.2) are the laser source, a Nd: $\mathrm{YVO}_{4}$ laser (Coherent Paladin, $1064 \mathrm{~nm}$ and $532 \mathrm{~nm}$ ), and a synchronously-pumped OPO (APE Levante Emerald). This combination provides a well-controlled and stable local oscillator as shown in chapter 3. The input powers of the fundamental and idler are several tens of $\mathrm{mW}$, while the power of the local oscillator is only a few $\mathrm{nW}$. The setup used for the heterodyne phase detection scheme (figure 4.1(a)) is almost the same as the one used in section 3.5. Heterodyne interferometric detection is obtained by modulation of the fundamental by an acousto-optical modulator (Crystal Technology 97-02848-01), (AOM 1, first order) resulting in a modulated CARS signal. By detecting the laser repetition rate and using a voltage controlled oscillator (VCO), an external frequency of $50 \mathrm{kHz}$ is added to the detected laser repetition rate and applied to the acousto-optical modulator. This $50 \mathrm{kHz}$ shift is translated to a $100 \mathrm{kHz}$ shift at the CARS wavelength (two fundamental photons in the CARS process). The CARS signal is detected on 


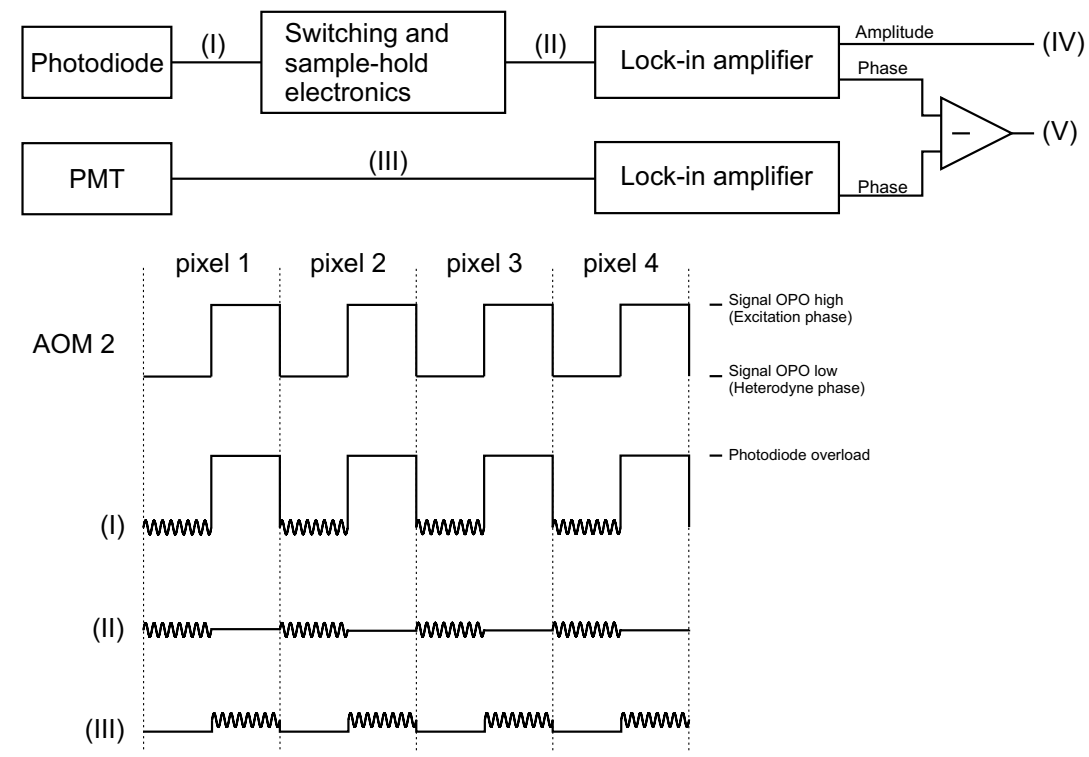

Figure 4.3: Electronic signal processing scheme to obtain the vibrational amplitude and phase. The output signals (IV) and (V) are respectively the vibrational amplitude and phase. AOM 2 shows the switching of the signal of the OPO, (I) detected Photodiode signal, (II) Photodiode signal after switching and sample-hold electronics and (III) PMT signal.

a photodiode (shot noise limited, see section 3.3) and fed to a lock-in amplifier (Stanford Research SR830) set to detect at $100 \mathrm{kHz}$.

For detection of the local excitation phase (figure 4.1(b)), the amplitude of the signal of the OPO must be changed from a few $\mathrm{nW}$ to the same level as the fundamental beam to balance the contribution of both pathways, route 1 and route 2 . The resulting interference between both routes is probed by the signal of the OPO and detected on a PMT (or photodiode).

To overcome interferometric instabilities between the two phase detection methods, an alternated phase detection on a point-by-point basis is added to the setup. Switching between the two power levels is done by a second acousto-optical modulator (AOM 2) in the OPO signal branch. This acousto-optical modulator is also used in first order (to obtain the necessary high extinction ratio), but driven at the laser repetition rate to prevent frequency modulation. The amplitude modulation frequency $(\sim 1 \mathrm{kHz})$ of AOM 2 is set to the frequency of the galvano scanners in the 
microscope. Scanning is done at $1 \mathrm{~ms} /$ pixel, alternating between the two different phase detection schemes. First the heterodyne phase is detected on the photodiode, when AOM 2 passes only a few $\mathrm{nW}$, and second the local excitation phase is detected on the PMT, when AOM 2 passes all OPO-signal power, see figure 4.3. With switching and sample-hold electronics the overload during the excitation phase detection time on the photodiode is removed. The detection of the local excitation phase is done by a second lock-in amplifier set to detect at the same $100 \mathrm{kHz}$ modulation. Both lock-in amplifiers are set to $1 \mathrm{~ms}$ integration time. Shorter pixel dwell times can be obtained by higher external modulation frequencies and shorter integration times.

A demonstration of the vibrational phase detection is shown in figure 4.4, where a sample fat globules (mayonnaise) of different sizes distributed in agarose gel is imaged. The phase of the heterodyne signal contains the vibrational phase of the sample, but the vibrational phase is hard to distinguish (due to the refractive index changes in the sample, phase curvature over the field of view and interferometric instabilities). The excitation phase also shows the phase disturbances, but not the vibrational phase. The subtraction of the excitation phase from the heterodyne phase yields the vibrational phase of the sample, without disturbances. In the vibrational phase image, phase steps can be seen on the location of the fat globules.

\subsubsection{Imaging of HeLa cells}

To demonstrate the capability of this phase detection scheme, further referred to as "Vibrational Phase Contrast CARS" or VPC-CARS [66], HeLa cells are imaged. Figure 4.5 shows fixed HeLa cells, in water, that contain lipid droplets of various sizes. CARS images of $50 \times 50 \mu \mathrm{m}$ (262x262 pixels), taken with a $1.2 \mathrm{NA}$ water objective, are acquired at $2845 \mathrm{~cm}^{-1}$. The heterodyne phase, figure $4.5(\mathrm{a})$, shows that the phase over the field-of-view is not constant, but distorted and noisy. At the location of the lipids in the cell we can see phase steps. These phase steps are mostly due to the difference in phase between the resonant and non-resonant signals. Figure 4.5(b) shows the local excitation phase detected on the PMT which is also distorted and noisy. Phase steps are observed at the location of the lipid droplets, but these steps are caused by the differences in refractive indices of the lipids and the water in the cell. The amplitude image of the CARS signal is shown in figure 


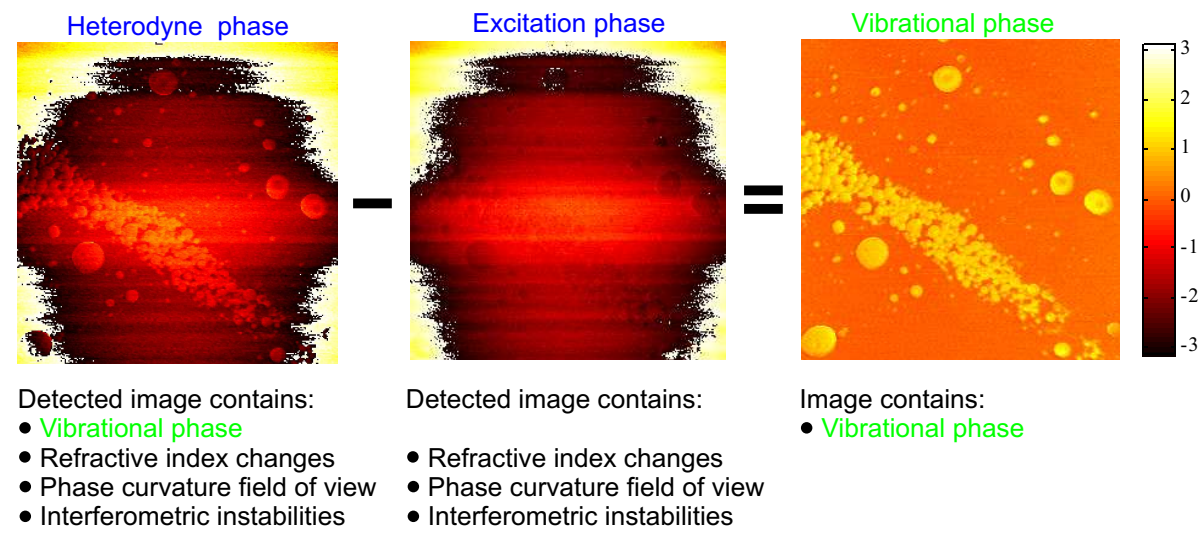

Figure 4.4: A sample of fat globules (mayonnaise) of different sizes distributed in an agarose gel solution imaged at $2865 \mathrm{~cm}^{-1}$. The subtraction of the heterodyne phase from the excitation phase shows a clean vibrational phase, without phase disturbances of the refractive index, curvature of field-of-view and interferometric instabilities.

4.5(c). There is contrast between the cells and the surrounding water, and the internal structure within the cells can be observed despite the lack of resonant material there. Resonant lipid droplets can easily be seen, as the amplitude of many droplets exceeds the non-resonant signal. Interferences on the edges of the droplets yield a "dark" ring around the droplets, enhancing the contrast. Note that there are also droplets represented by dark spots in the amplitude image. Subtraction of the local excitation phase from the heterodyne phase can be seen in figure $4.5(\mathrm{~d})$. The distortion and noise cancel out, allowing for averaging of this vibrational phase image to increase the precision on the phase value.

In this image the phase of the pure non-resonant water signal outside the cell is used to pinpoint phase zero and subtract a residual phase difference that is caused by the AOM 2 switching between different levels. Since there is a strong non-resonant background, the phase step at the lipids does not reach $\pi / 2$, see figure $4.5(\mathrm{f})$, line cross section (d). Equation 3.5 shows that taking the sine of the vibrational phase image and multiplying it with the amplitude image, removes the non-resonant signal, which is done in figure 4.5(e). Comparing the amplitude image and the background free image, it can be seen that the internal structure of the cell and the edge of the cell have disappeared and only the resonant lipids can be observed. This shows that the cell structure in the amplitude image is non-resonant and should not be confused for the 


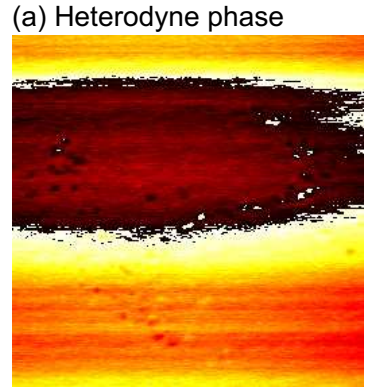

(c) Amplitude

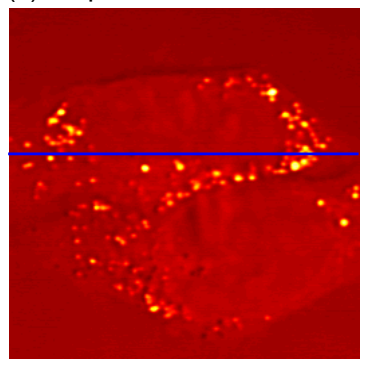

(e) Background free

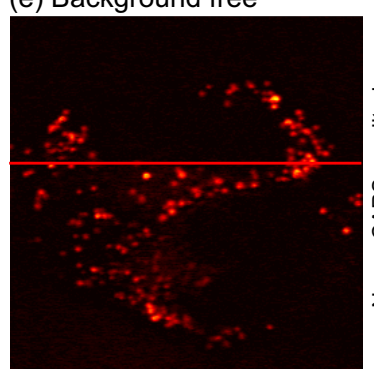

(b) Excitation phase

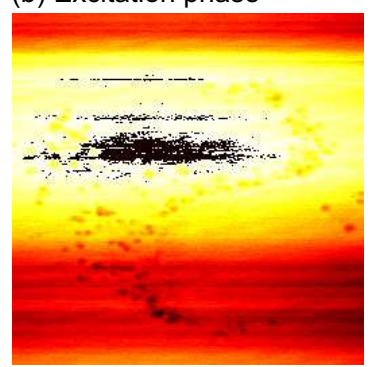

(d) Vibrational phase
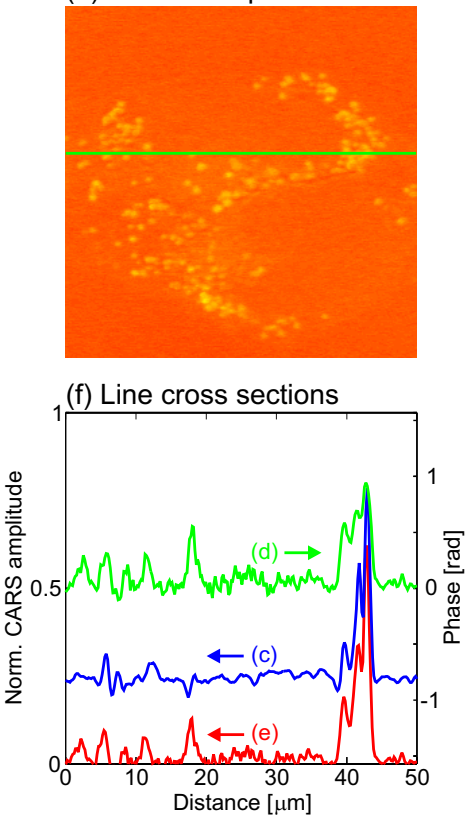

Figure 4.5: Fixed HeLa cells, in water, with various sizes of lipid droplets imaged at the vibrational stretch of $2845 \mathrm{~cm}^{-1}$. Heterodyne phase (a) and local excitation phase (b). Average of five measured images of CARS amplitude (c) and vibrational phase (d) (subtraction of the local excitation phase from the oscillator phase). (e) Background free amplitude. (f) Line cross sections of images $c, d$ and $e$. The scaling of the phase images $(a, b$ and $d)$ is the same. The scaling of the amplitude images ( $c$ and $e$ ) is also the same.

resonant material at the cell boundary. The line cross section, see figure $4.5(\mathrm{f})$, shows that the non-resonant amplitude is lowered from 0.25 to less than 0.02. The three lipid droplets in the line cross section around $42 \mu \mathrm{m}$ show only a small drop in amplitude due to the $\sim \pi / 4$ phase 


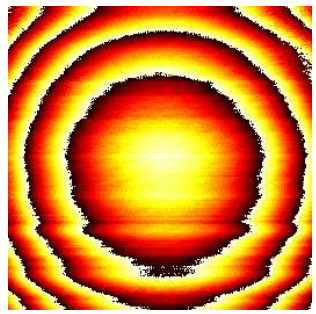

$3055 \mathrm{~cm}^{-1}$

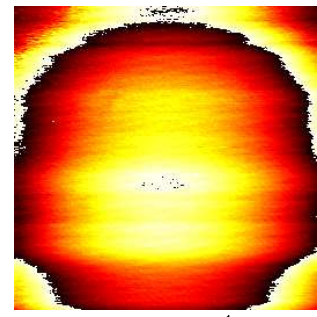

$1605 \mathrm{~cm}^{-1}$

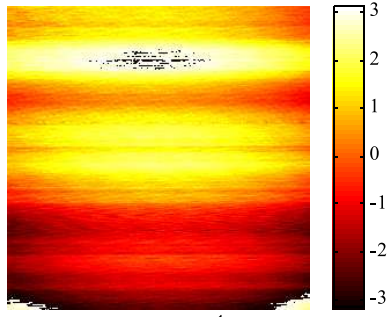

$1003 \mathrm{~cm}^{-1}$

Figure 4.6: Change of phase curvature of the field of view shown for three different resonances of toluene by detection of the local excitation phase.

step at these locations. The region between 0 and $15 \mu \mathrm{m}$ shows lipid droplets that are faint compared to the non-resonant signal, but stand out when the non-resonant signal is removed. The dispersive feature at $18 \mu \mathrm{m}$, is also accompanied by a phase step, resulting in a recovered clean amplitude in the background free image.

\subsubsection{VPC-CARS in the fingerprint region}

When tuning the VPC-CARS setup toward the fingerprint region, the shift in CARS wavelength results in the need for a different detector for the detection of the local excitation wavelength is improved at this wavelength. The quantum efficiency of the photodiode for the heterodyne detection is getting better, but the quantum efficiency of the PMT (Hamamatsu R1463) for the excitation phase detection drops dramatically and it has to be replaced by a IR-sensitized PMT (Hamamatsu R943-02) or by a second photodiode. Although the photodiode is limited by its high dark noise the generation of the local excitation CARS signal is sufficiently strong to detect the CARS signal with its modulation on a photodiode. Detection of vibrational resonances in the fingerprint region results in input beams that are spectrally closer together. As a direct effect the phase curvature of the field of view contains less fringes. In figure 4.6 the phase of the excitation field is shown for three different vibrational resonances of toluene showing this change of phase curvature of the field of view.

Detection of lower frequency vibrational resonances gives the opportunity to study biological samples in the congested, informative, fingerprint region. In biological systems the resonant signal from this finger- 
(a) Amplitude

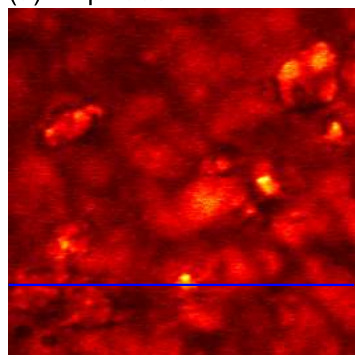

(c) Background free

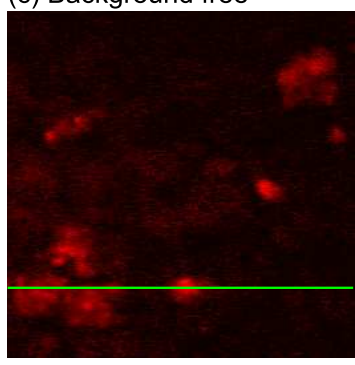

(b) Vibrational phase
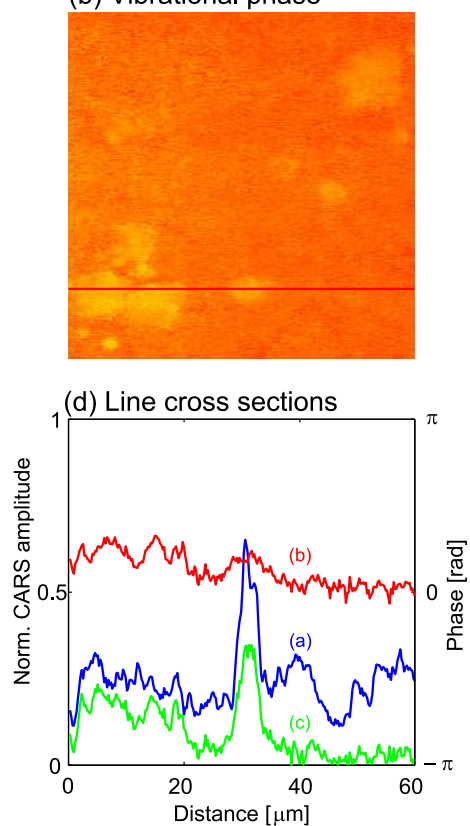

Figure 4.7: $V P C-C A R S$ image at $950 \mathrm{~cm}^{-1}$ of bone growing cells.

print region is normally overwhelmed by the non-resonant background signal. In figure 4.7 a demonstration of VPC-CARS is given on cells that are capable of growing bone. A detailed study of the bone growth from these cells can be found in section 5.3. Figure 4.7 is imaged at 950 $\mathrm{cm}^{-1}$, a vibrational resonance for the label-free detection of early bone mineralization. In the amplitude image a large non-resonant background characterizes the image, but the vibrational phase image shows several locations of different phase. These locations result in the the resonant bone in high signal to background visibility of early Calcium phosphate minerals typical for developing bone constituents. 


\subsection{Third order cascaded phase-preserving chain}

\subsubsection{Single wavelength CARS detection}

In 1997, Chen [67] demonstrated CARS spectroscopy measured at a single (constant) wavelength over the entire vibrational spectrum. In this study the third harmonic $(355 \mathrm{~nm})$ of a Nd:YAG laser $(1064 \mathrm{~nm})$ is synchronously pumping an OPO. CARS spectra are obtained by using the OPO idler as pump wavelength, fundamental of the laser as Stokes wavelength and OPO signal as probe wavelength. The generated CARS signal results in the double fundamental wavelength $(532 \mathrm{~nm})$ and has the same wavelength over the full scanning range. This results in the ability to measure a complete spectrum by tuning the OPO, but with detection at a single wavelength. Chen used this single wavelength detection for efficient background rejection in spectra obtained in the presence of high background levels, as in remote sensing and atmospheric studies. Furthermore, due to the single wavelength detection, more efficient and specialized filters and detectors can be used.

\subsubsection{The cascaded phase-preserving chain}

In section 3.2.2 the cascaded phase-preserving chain is explained and it us shown how the OPO signal can interfere with the generated CARS signal. The phases and wavelengths are locked to each other and allow for amplitude and phase detection of the generated CARS signal. The cascaded phase-preserving chain uses an OPO that is pumped by the second harmonic of the laser, see figure 4.8(a). Table 4.1 shows the wavelength and phase relations in the different stages of the cascaded phase-preserving chain. The excitation phase is further obtained by using the interference between the two routes addressing a vibrational resonance or virtual state, see figure 4.8(b). Switching between the heterodyne phase detection scheme and excitation phase detection scheme results in the VPC-CARS, as shown in chapter 4, where the phase of the oscillators in the focal volume is measured.

The single wavelength detection scheme of Chen can be used in the VPC-CARS detection scheme. This section will explain how to implement the single wavelength detection scheme, how the phase is preserved and what the advantages of this detection scheme are. 


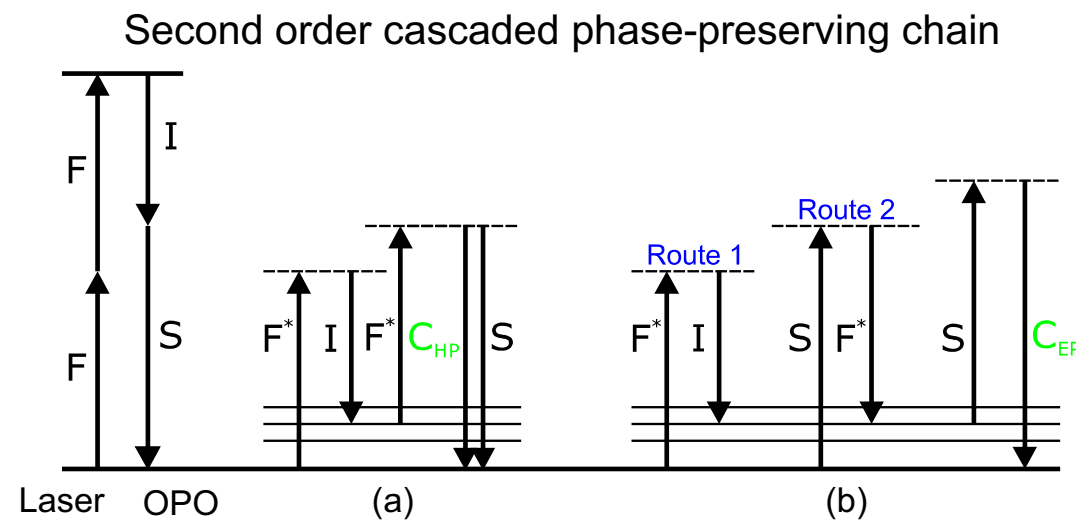

Figure 4.8: Schematic of the second order cascaded phase-preserving chain between laser, OPO and CARS process. Where (a) shows the heterodyne phase detection scheme and (b) shows the local excitation phase detection scheme. $F=$ fundamental laser, $F^{*}=$ modulated fundamental laser, $I=O P O$ idler, $S=O P O$ signal and $C=C A R S$ signal.

\begin{tabular}{|l|l|l|}
\hline Process & Wavelength relation & Phase relation \\
\hline 1. SHG & $2 \cdot \omega_{F}=\omega_{2 F}$ & $2 \cdot \phi_{F}=\phi_{2 F}$ \\
2. OPO & $\omega_{2 F}=\omega_{S}+\omega_{I}$ & $\phi_{2 F}=\phi_{S}+\phi_{I}$ \\
3. CARS & $\omega_{C A R S}=2 \cdot \omega_{F}-\omega_{I}$ & $\phi_{C A R S}=2 \cdot \phi_{F}-\phi_{I}+\phi_{\chi^{3}}$ \\
4. PPC & $\omega_{C A R S}=\omega_{S}$ & $\phi_{C A R S}=\phi_{S}+\phi_{\chi^{3}}$ \\
\hline
\end{tabular}

Table 4.1: The table shows the wavelength and phase relations of the individual stages in the second order cascaded phase-preserving chain.

Figure 4.9 shows schematically how the signal and idler from the OPO are created by the third harmonic of the laser. Heterodyne CARS is obtained with the OPO idler as pump wavelength, the laser fundamental as Stokes wavelength and the OPO signal as probe wavelength. The generated CARS wavelength is at a single wavelength and is independent of tuning of the OPO. Furthermore it has the same wavelength as the second harmonic of the laser, see figure 4.9(a). In table 4.2 the wavelength and phase relations are shown of the third order phase-preserving chain. It can be seen that also in this creation process the wavelength and phases of the generated CARS signal and second harmonic of the laser are locked to each other and allowing them to be interfered with each other. 


\section{Third order cascaded phase-preserving chain}

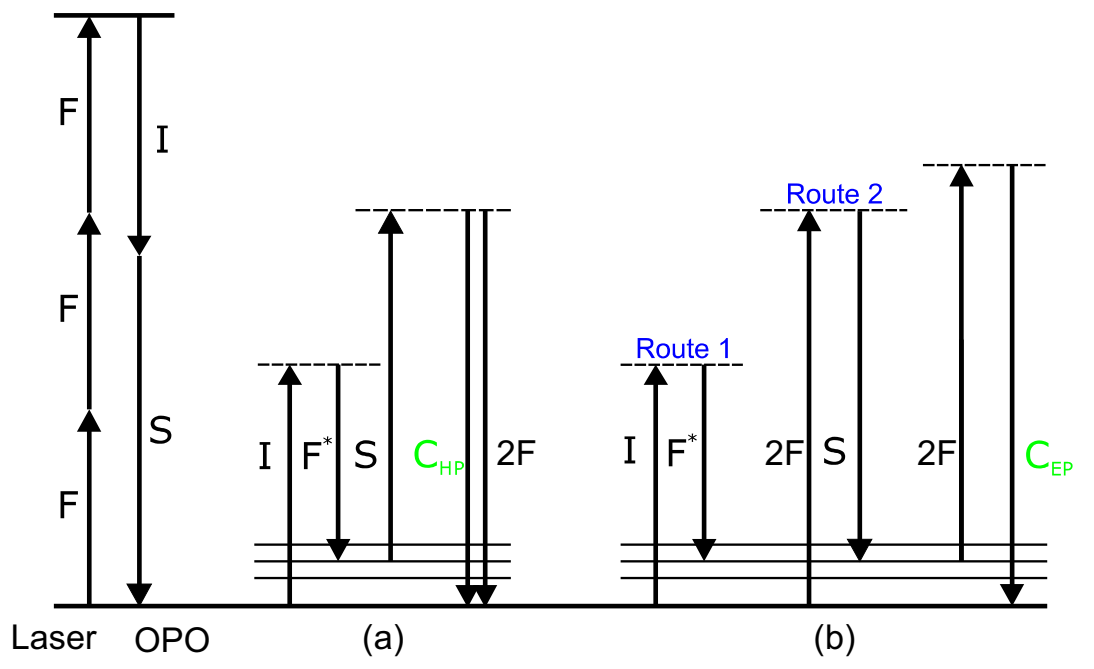

Figure 4.9: Schematic of the third order cascaded phase-preserving chain between laser, OPO and CARS process. Where (a) shows the heterodyne phase detection scheme and (b) shows the local excitation phase detection scheme. $F=$ fundamental laser, $F^{*}=$ modulated fundamental laser, $2 F=$ second harmonic of the laser, $I=O P O$ idler, $S=O P O$ signal and $C=C A R S$ signal.

\begin{tabular}{|l|l|l|}
\hline Process & Wavelength relation & Phase relation \\
\hline 1. SHG & $2 \cdot \omega_{F}=\omega_{2 F}$ & $2 \cdot \phi_{F}=\phi_{2 F}$ \\
2. THG & $3 \cdot \omega_{F}=\omega_{3 F}$ & $3 \cdot \phi_{F}=\phi_{3 F}$ \\
3. OPO & $\omega_{3 F}=\omega_{S}+\omega_{I}$ & $\phi_{3 F}=\phi_{S}+\phi_{I}$ \\
4. CARS & $\omega_{C A R S}=\omega_{I}-\omega_{F}+\omega_{S}$ & $\phi_{C A R S}=\phi_{I}-\phi_{F}+\phi_{S}+\phi_{\chi^{3}}$ \\
5. PPC & $\omega_{C A R S}=\omega_{2 F}$ & $\phi_{C A R S}=\phi_{2 F}+\phi_{\chi^{3}}$ \\
\hline
\end{tabular}

Table 4.2: The table shows the wavelength and phase relations of the individual stages in the third order optical phase preserving chain.

Detection of the excitation phase is achieved by the combination of route 1 , OPO idler minus fundamental, and route 2 , second harmonic of the laser minus OPO signal. In this case the CARS signal is generated by probing the interference with the second harmonic of the laser, see figure $4.9(\mathrm{~b})$. 


\subsubsection{Differences in detection}

\section{Modulation frequency}

In the second order cascaded phase-preserving chain (CPPC) the fundamental wavelength is frequency modulated to allow detection of the interference between the generated CARS signal and the OPO signal wavelengths, which enables CARS amplitude and phase detection. The beating frequency is doubled in respect to the modulation frequency due to the fundamental contributing twice in the CARS generation process. In the excitation phase detection scheme the fundamental is positive in route 1 and negative in route 2 , resulting in twice the modulation frequency as the detected beating frequency.

In the third order CPPC the fundamental can still be modulated. This modulation will result in a CARS signal at the beating frequency in both the excitation detection scheme and the heterodyne detection scheme, but now the interference appears at the beating frequency. In this case the acoustic optical modulator (AOM) should be giving pure frequency modulation and absolutely no amplitude modulation. The second harmonic of the laser can also be modulated, resulting in the same beating frequency as the frequency modulation of the fundamental.

\section{Wavelength detection}

The second and third order CPPCs result in different wavelength detection throughout the vibrational spectrum. In figure 4.10 graphs are shown for two different scenarios. The second order CPPC is based on an OPO pumped at the second harmonic wavelength of a $1064 \mathrm{~nm}$ laser, figure 4.10(a). And the third order CPPC is based on a OPO pumped at the third harmonic wavelength of a $1500 \mathrm{~nm}$ laser (telecom wavelengths), figure 4.10(b). In both scenarios a LBO based OPO, pumped around $500 \mathrm{~nm}$ is selected, because these are commercial available. The $1064 \mathrm{~nm}$ laser is also commercially available. However, a $1500 \mathrm{~nm}$ picosecond laser with enough power in the third harmonic to efficiently pump an OPO is not yet available. For this simulation a $1500 \mathrm{~nm}$ laser is chosen, instead of the third harmonic of $1064 \mathrm{~nm}$ laser. To limit the CARS frequencies and prevent the associated problem such as increased tow photon absorption and cell damage and avoid two-photon enhanced non-resonant background.

From figure 4.10 it can be seen that the heterodyne CARS (H-CARS) signal in the third order CPPC has a constant wavelength and the local 
excitation phase CARS (LEP-CARS) signal is always lower in wavelength. In particular

$$
\lambda_{F}>\lambda_{O P O_{\text {idler }}}>\lambda_{O P O_{\text {signal }}}>\lambda_{H-C A R S}>\lambda_{L E P-C A R S} .
$$

This results in a single optimized filter set in combination with matching detector for the detection of the H-CARS and LEP-CARS signal, which is not possible for the second order cascaded phase-preserving chain, where multiple filter sets are needed to cover the full vibrational spectrum.

Furthermore the cavity mirror set of the OPO determines how close it can be tuned toward the degeneracy point - the crystal temperature where the OPO signal is at the same frequency as the OPO idler. In the case of the second order cascaded phase-preserving chain, being unable to tune close toward the degeneracy point will exclude the lowest frequency vibrational resonances from the covered vibrational range. The third order CPPC will exclude the highest frequency vibrational resonances. CARS microscopy has proven its use in the high frequency range, but in the future the lower frequency range expected to become more important to improve chemical selectivity. The lower frequency range shows more characteristic frequencies that typical can be used to identify specific molecules.

\subsubsection{Conclusion}

When a laser source becomes commercially available for effective pumping of the OPO with the third harmonic of the laser (around $500 \mathrm{~nm}$ ), the third order cascaded phase-preserving chain can be used. This method based on the VPC-CARS and single wavelength detection schemes could attract more commercial interest, due to its background free imaging capabilities over the full vibrational spectrum and its simplicity for the user. The filters and detectors do not need to be changed and can be designed specifically for this application. 

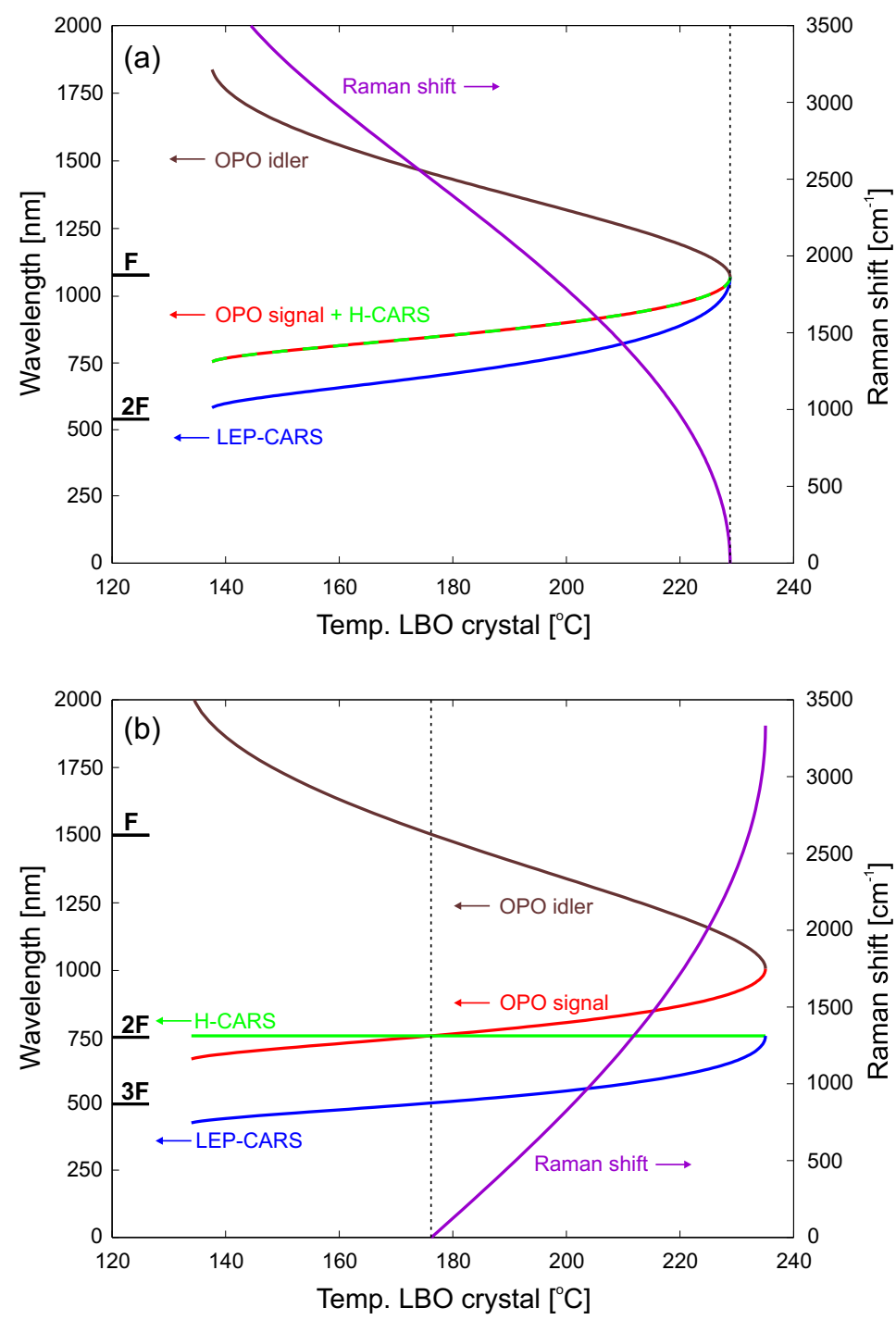

Figure 4.10: Graphs show the OPO signal and idler wavelength for a certain crystal temperature on the left wavelength axis. The generated heterodyne CARS (H-CARS) and local excitation phase CARS (LEP$C A R S)$ wavelengths at a certain crystal temperature also on the left wavelength axis. The addressed Raman shift at a certain crystal temperature are displayed on the right Raman shift axis. (a) Second order $C P P C$ and (b) third order CPPC. F, $2 F$ and $3 F$ are respectively the fundamental, second harmonic and third harmonic wavelength of the laser. 


\subsection{Detection in the complex plane}

\subsubsection{The complex plane}

For a given driving frequency, the molecules in the focal volume emit a combined amplitude and phase, that can be represented by one point in the complex plane defined by its vector length and angle, see figure 4.11. A single resonance traces a circle in the complex plane as a function of frequency. Multiple resonances result in a circle with additional circular features. In figure 4.11 the amplitude, phase and complex plane representation are shown of the resonances of polyethylene around 3000 $\mathrm{cm}^{-1}$, derived from figure 3.12. The spectrum of polyethylene shows four resonances, where the strength of the resonance and the associated phase determine the shape of the features in the complex plane. The offset on the real axis represents the level of non-resonant background.

Detection of the vibrational phase of the oscillators in the focal volume is possible with VPC-CARS, without the need for a reference phase in the sample, as was used to obtain the spectrum of polyethylene in figure 3.12. This VPC phase detection allows for recording of background free spectra in a single point. In figure 4.12 the spectrum of a volume mixture of $50 \%$ ethanol and $50 \%$ water is recorded. To record this spectrum, the Lyot filter in the OPO is (automatically) rotated, tuning the signal wavelength over $12.3 \mathrm{~nm}$. This signal scan corresponds to a spectrum over $187 \mathrm{~cm}^{-1}$. Every point in the spectrum is measured in 0.5 seconds, resulting in a total measuring time of 26 seconds. Note that the beam displacement caused by the grating in AOM 2 (which give rise to amplitude modulation) is negligible within one Lyot filter rotation.

By extending the complex plane with a third axis for the driving frequency of the molecule, a spiral or corkscrew in complex space can be constructed, see figure 4.13. Looking along the frequency axis (front view) shows the complex plane. Looking along the the imaginary axis (bottom view) the real plane is obtained, that shows the typical dispersion caused by wavelength-dependent refractive index changes. The last view in this $3 \mathrm{D}$ plane is along the real-axis (side view) and shows the imaginary plane, with the absorption profile of the molecule which is directly related to the Raman intensity spectrum. From the 3D perspective it is clear that some resonances that seem to overlap in amplitude can have significant differences in phase. 

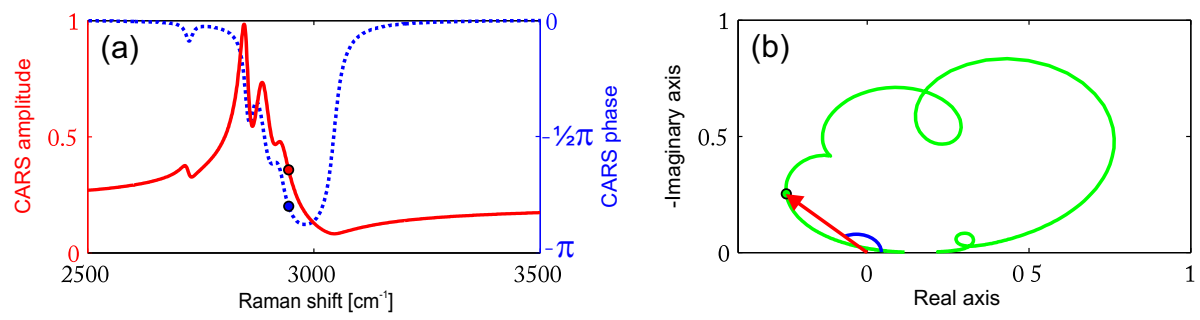

Figure 4.11: (a) Amplitude (red) and phase (blue) of the resonances of polyethylene around $3000 \mathrm{~cm}^{-1}$. (b) The amplitude and phase are constructed in the complex plane. The CARS amplitude vector is shown in red and the phase angle is shown in blue.
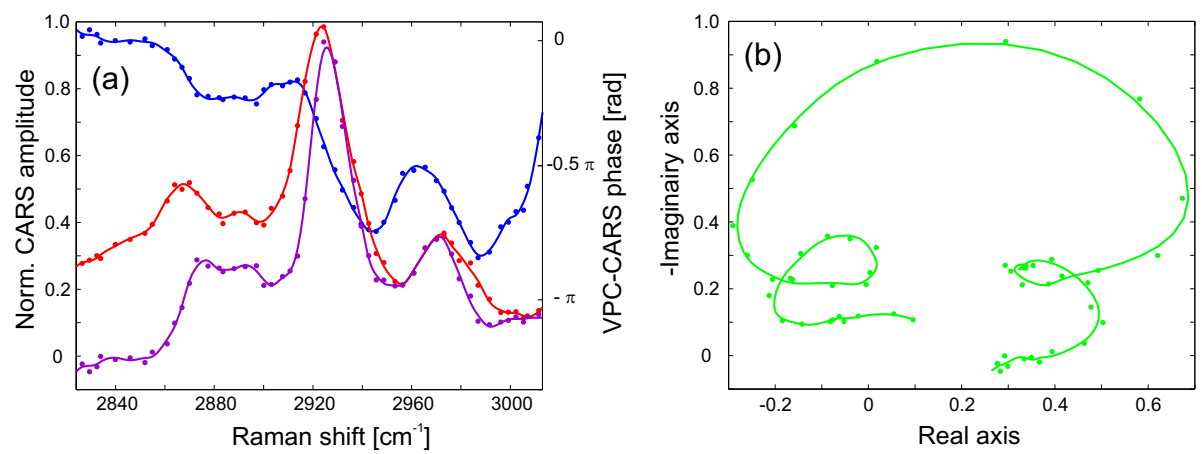

Figure 4.12: (a) The CARS spectrum (red), VPC-CARS phase (blue) and background free spectrum (purple) of a volume mixture of $50 \%$ ethanol and $50 \%$ water. (b) The CARS amplitude and VPC-CARS phase are constructed in the complex plane.

A measured signal is the combined result of all the amplitudes and phases of the molecules in the focal volume. Within the focal volume the signal from these molecules is coherently added. When the focal volume contains only one type of molecule we obtain constructed points as shown in (I) and (III) in figure 4.14, where (III) has a stronger vibrational stretch than (I). A mixture of different molecules will result in a addition of the individual molecules with their specific vector length and angle. For example, a 50/50\% molecular mixture of the two types of molecules will result in the distribution as displayed in (II), containing half of the (I) molecules, with their associated vector length and angle and half of the (III) molecules. 

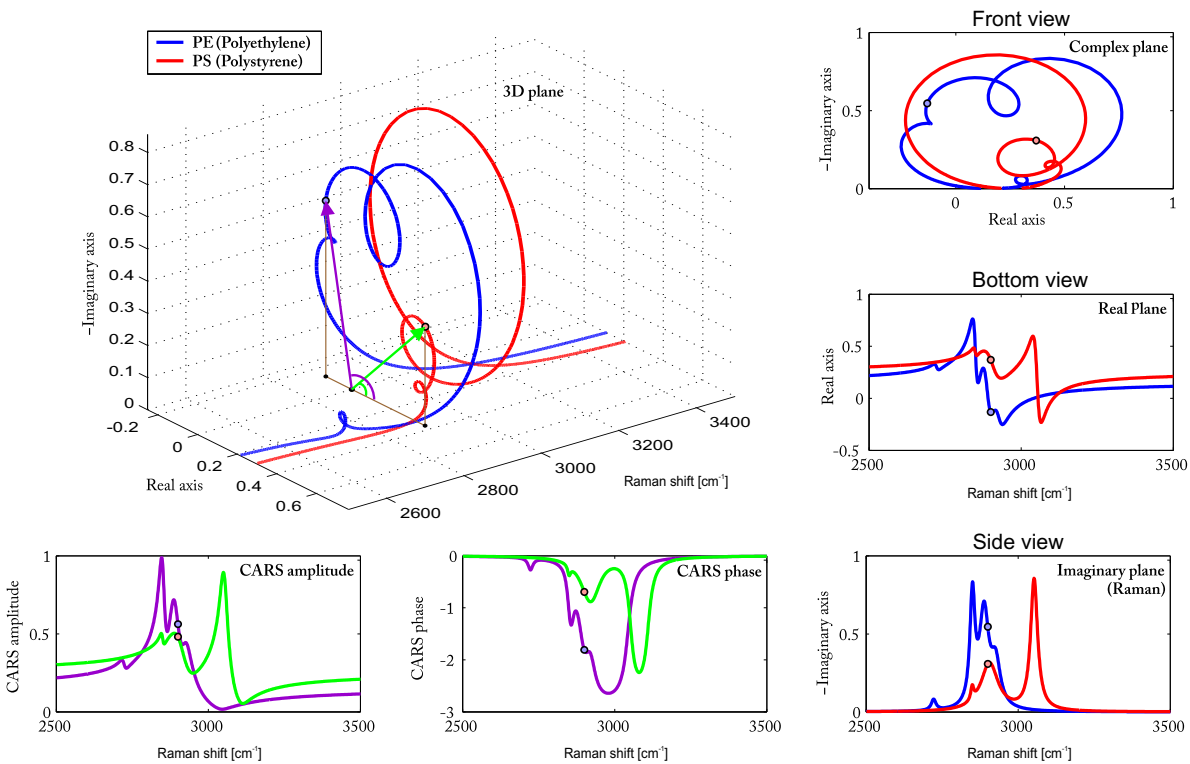

Figure 4.13: "The molecular rollercoaster" of polyethylene (PE) and polystyrene (PS), where the position in the complex plane is plotted against the frequency. The three different views of this $3 D$ space are on the right side. The amplitude and phase of the CARS signal are plotted in the bottom-left.

The changes in amplitude and phase can be used to monitor a sample or system over time. A dissolution graph, where a resonant compound is dissolved in a non-resonant compound, can be seen in figure 4.14(IV). When two partially resonant compounds are mixed together we obtain figure $4.14(\mathrm{~V})$. Figure $4.14(\mathrm{~V})$ can also be obtained when a reaction between two molecules or transformation of a molecule (crystalinity, folding) is monitored over time. When two molecules react via an intermediate state, figure 4.14(VI), could be obtained. These are just a few theoretical examples of the possibilities of monitoring molecular dynamics in the complex plane.

\subsubsection{Monitoring dissolution in the complex plane}

An example of a dilution graph is given in figure 4.15(a). This graph is obtained at the vibrational resonance of $2927 \mathrm{~cm}^{-1}$. Ethanol (resonant) is mixed in different ratios with water (non-resonant). Eleven samples, ranging from pure ethanol to pure water with $10 \%$ volume ratio inter- 

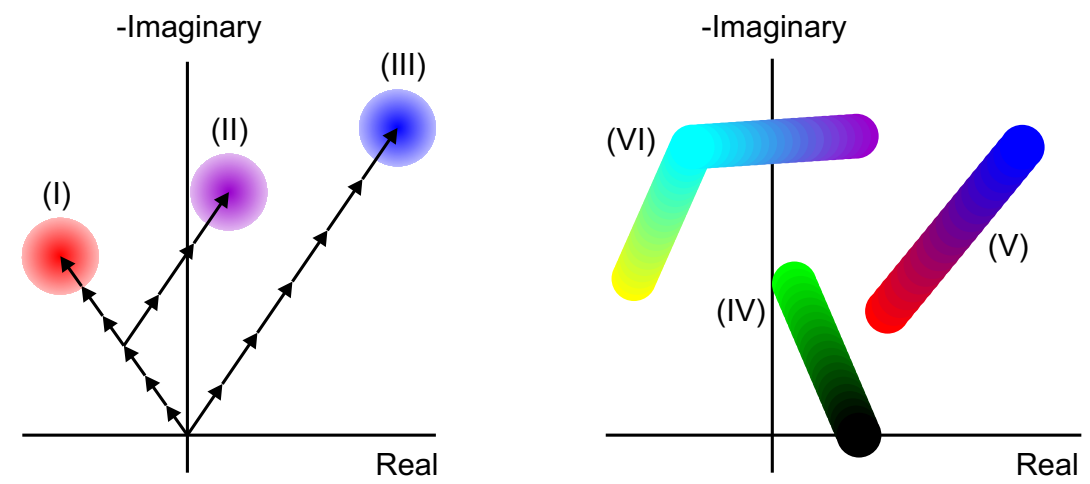

Figure 4.14: Complex plane trajectories obtained at one vibrational resonance. Left: compound (I) and (III) are constructed contain the same number of molecules but have a different angle and vector length. A $50 / 50 \%$ molecular mixture between (I) and (III) gives location (II) in the complex plane. Right: (IV) shows the dilution of a resonant compound, $(V)$ shows a mixing or reaction between two resonant compounds and (VI) shows a reaction via an intermediate state.
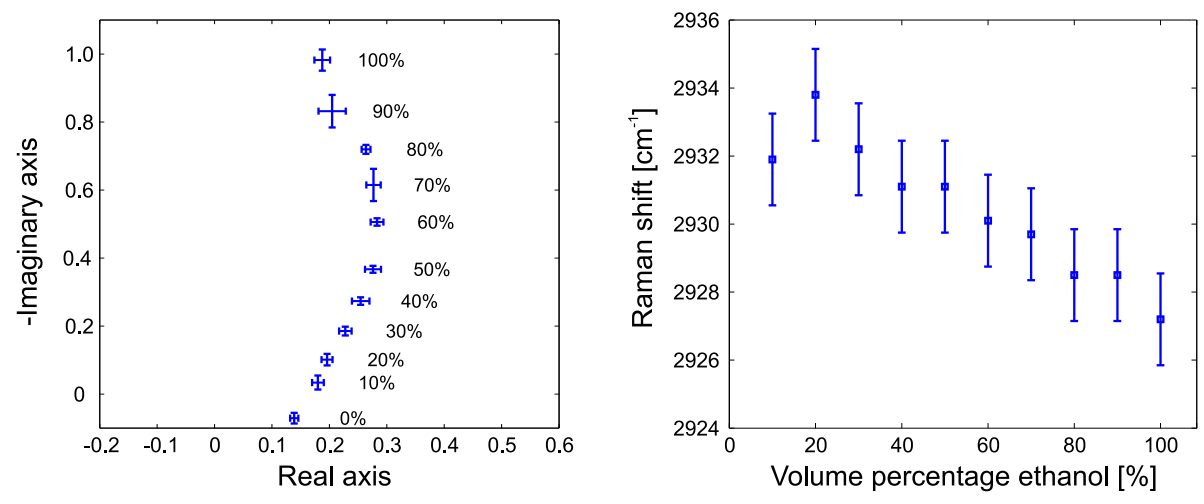

Figure 4.15: Dilution of ethanol in water as function of volume percentage.

vals, are flow through a cell. The dilution does not follow a straight line between the pure substances, but curves. This curvature is consistent with the Raman scattering data on the location of the resonance peak of ethanol as a function of the mixing ratio with water, see figure 4.15(b). It can be seen that the peak position shifts towards higher Raman frequencies when the ethanol is diluted with water. This shift also results in a shift of the associated vibratioal phase, decreasing the angle while diluting. This experiment shows that the interaction dynamics between 

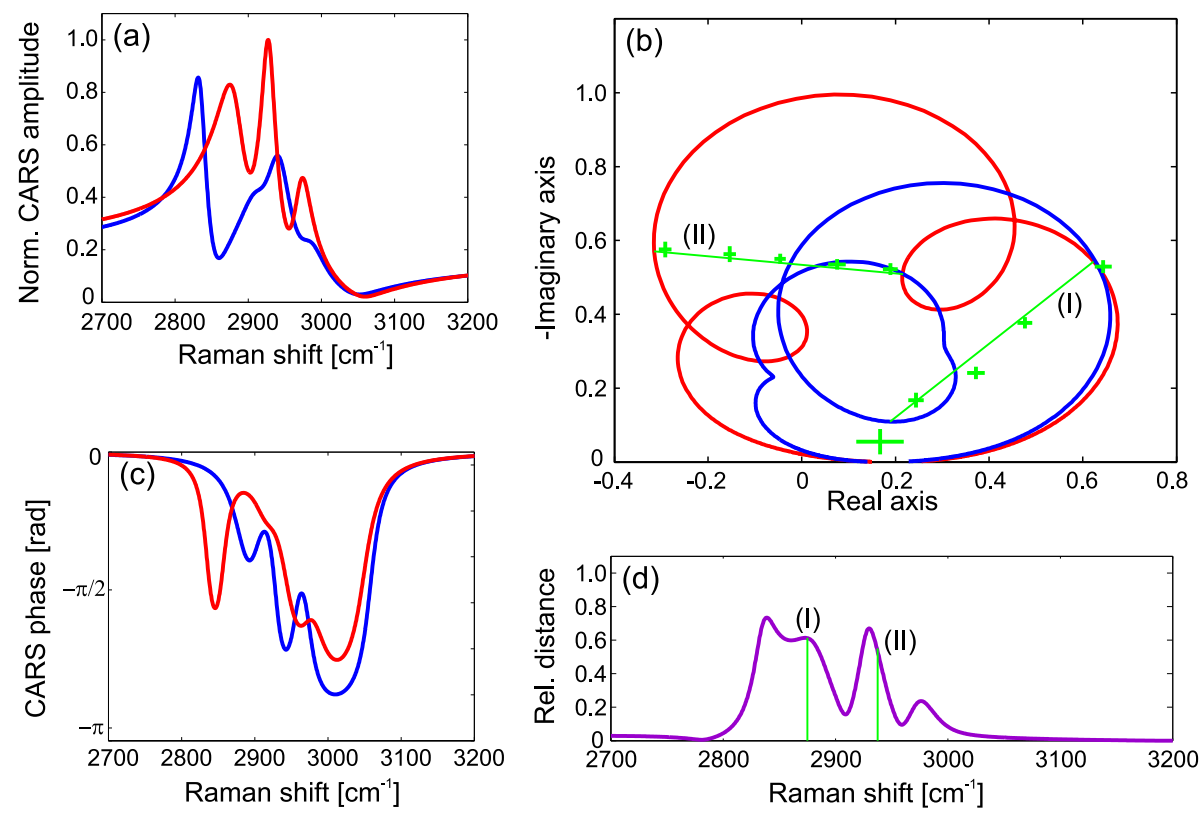

Figure 4.16: $C A R S$ amplitude (a), phase (b) and complex representation (c) of ethanol (blue) and methanol (red). (d) Shows the relative distance between the complex points at the vibrational resonances, where (I-II) are measured locations. The green line and data points show the measured mixing points and theoretical line at 2871 and $2937 \mathrm{~cm}^{-1}$.

ethanol and water are more complicated than just a combination of the two compounds.

\subsubsection{Monitoring mixing in the complex plane}

Mixing of two compounds that are both resonant result in different pathways trough the complex plane, depending on the frequency that is used (see figure $4.14(\mathrm{~V})$. The distance between the positions of pure compounds in the complex plane can be changed by measuring at a different vibrational resonance. Based on Raman spectra of the pure substances, the trajectories of ethanol and methanol are calculated (figure 4.16). The length of the difference vector as function of frequency is shown in figure 4.16(d). In the experiment ethanol and methanol are mixed in five steps, from pure ethanol to pure methanol with $25 \%$ volume percentage mixing steps. The mixtures, measured at $2936 \mathrm{~cm}^{-1}$, are represented in green in figure 4.16(b), showing a very good agreement with the theoretical line. 


\subsubsection{Multi component analysis}

All compounds have vibrational resonances. Some of these resonances overlap closely, others just partially. The differences in amplitude can be very small between the different compounds, but the difference in phase can be substantial. In figure 4.13 two plastics (PE and PMMA) have almost the same amplitude at the vibrational resonance of $2940 \mathrm{~cm}^{-1}$, but there is a phase difference of about 1 radian. Therefor the two plastics have a very different location in the complex plane at this frequency. This difference can be exploited to separate the compounds in an image.

In figure 4.17, a combination of three different materials is imaged with VPC-CARS at the vibrational frequency of $2940 \mathrm{~cm}^{-1}$. The sample contains a PE sheet on the left side, $4 \mu \mathrm{m}$ PMMA beads and water. The amplitudes of the PE and PMMA are almost the same at this frequency and the signal from the water is so weak that it can be considered as nonresonant background signal. In the phase image a clear difference can be observed between the PE and PMMA. When the amplitude and phase information of all points in the image are displayed as a density plot in the complex plane, it is possible to distinguish three different areas. The points in these three different areas can be used separately to show the three pure constituents. The elongated spread of the PMMA beads is probably caused by the spherical shape of the beads. Between the PE points and the PMMA and water points there is a crossing, showing the points where the focal volume consists of a mix of both compounds. These reconstructed images from the density complex plane are all free from background signal; they relate only to the signal from the pure substances. This example illustrates that VPC-CARS allows for multicomponent separation at a single vibrational resonance.

Separation of more components with increased amplitude and phase overlap can be obtained by extending the detection to multiple frequencies. The complex space is then constructed as in figure 4.13 and measurements at multiple frequencies can be obtained by automatic rotation of the Lyot filter as done for the spectrum in figure 4.12. This will yield elongated clouds of points in space. With prior knowledge of the trajectories in the 3D complex space, decompositions of more complex compounds can be made, further improving the selectivity of narrowband CARS microscopy. 

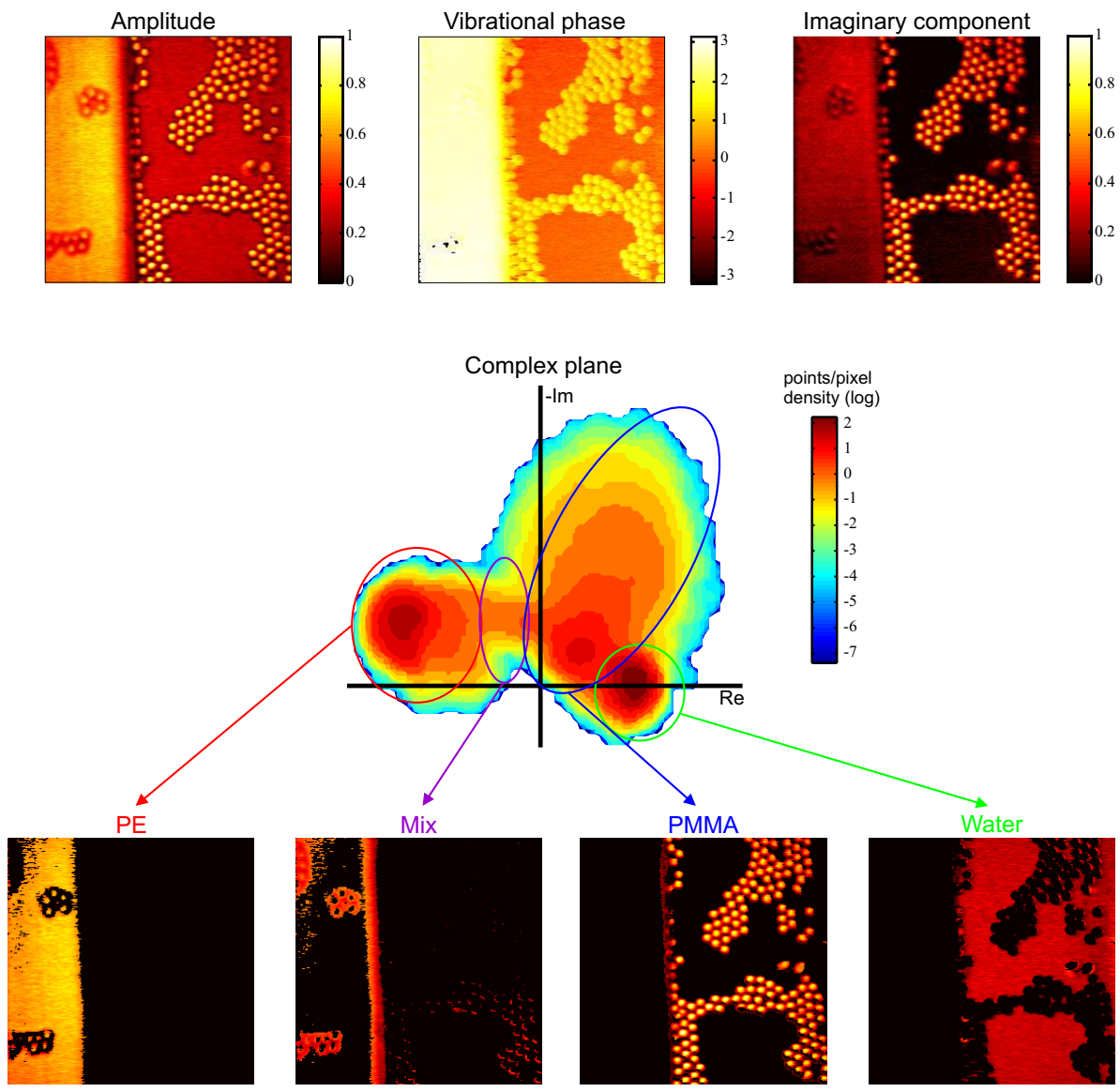

Figure 4.17: Top: amplitude, vibrational phase and background free image of a sample containing a sheet of PE, $4 \mu \mathrm{m}$ PMMA beads and water, imaged at $2940 \mathrm{~cm}^{-1}$. Middle: density graph of the projected amplitude and vibrational phase points in the complex plane. Bottom: multi component analysis, where the different components are separated by their location in the complex plane.

\subsection{Conclusion and Outlook}

\subsubsection{Conclusion}

VPC-CARS allows for detection of the CARS amplitude and phase. The subtraction of the local excitation phase from the heterodyne phase results in the vibrational phase of the oscillators in the focal volume. With VPC-CARS it is possible to obtain background free images over the full vibrational spectrum by projection of the CARS amplitude onto 
the imaginary axis, giving an image that is comparable to the Raman scattering intensity image. The background free detection capabilities are demonstrated by imaging of lipid droplets $\left(2845 \mathrm{~cm}^{-1}\right)$ in HeLa cells and bone structures $\left(950 \mathrm{~cm}^{-1}\right)$ of bone growing cells.

Furthermore a third order cascaded phase-preserving chain was presented that can simplify the detection and provide access of the low wavenumber region. The enabling factor for this technology would be the commercial availability of a laser source which is capable of efficiently pumping the OPO with the third harmonic of the laser (around $500 \mathrm{~nm}$ ).

VPC-CARS can be used to monitor dynamic processes in the complex plane. The detected CARS amplitude and phase represent a vector with an amplitude and angle in the complex plane. Dynamic processes such as dilution and mixing where demonstrated.

\subsubsection{Outlook}

The precision that was reached for recording a position in the complex plane, with an integration time of $250 \mathrm{~ms}$, was $\pm 1.1 \%$ standard deviation in amplitude and $\pm 2.0^{\circ}$ degrees in phase. Within a cubic micron in the order of a billion molecules are present, this gives as an precision in the order of a million molecules as minimal detectable amount. To increase the analytic abilities, the detection accuracy of amplitude and phase have to be improved. 


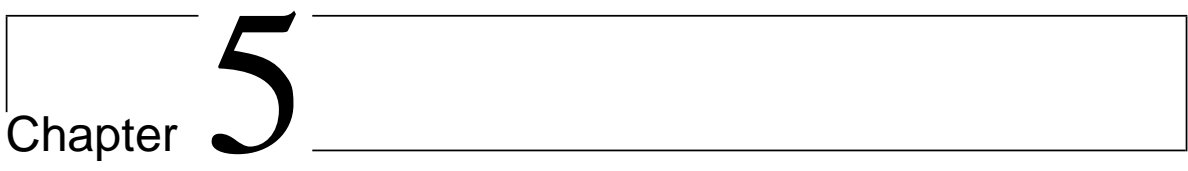

\section{Applications of CARS microscopy}

CARS microscopy is a powerful imaging technique for biology, pharmacology and the food industry, where chemical selectivity and label-free detection are essential. In CARS microscopy, the differences in the vibrational spectra of molecules are used to obtain specificity. Other nonlinear signals are generated simultaneously that can be exploited to obtain more information from the sample. In this chapter, CARS microscopy is used as one modality in a multimodal nonlinear microscopy setup. CARS, second harmonic generation microscopy and spontaneous Raman spectroscopy can be used, side by side. Two different studies are shown, one pharmaceutical and one biological, in which the combination of these techniques resolves questions on the dissolution behavior of a drug and how cells grow bone. 

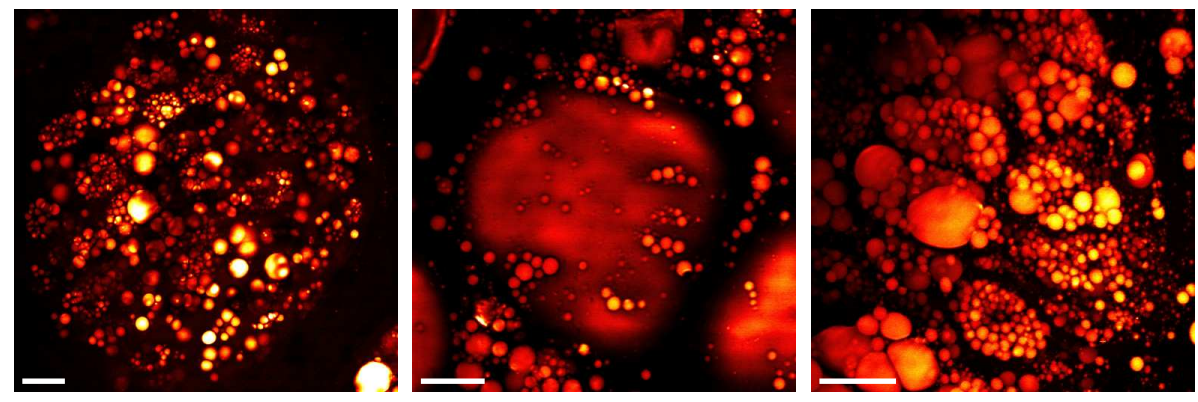

Figure 5.1: Images of different fat clusters in pork tissue, imaged at the vibrational resonance of lipids at $2845 \mathrm{~cm}^{-1}$. The scale bar is $20 \mu \mathrm{m}$.

\subsection{CARS as part of a nonlinear microscope system}

CARS microscopy has successfully demonstrated in vitro visualization of lipid droplets in cells [68] and monitoring of rapid organel transport [69]. It has also be used for in vivo studies of Caenorhabditis Elegans [70] and mice [71]. Furthermore, arteriosclerotic vascular diseases [72] and brain tumors [73] can be visualized ex vivo and chemical compounds can be detected in human hair [74]. These are just a few examples of the rapidly growing number of studies that use CARS microscopy. The variety in these studies demonstrates the diverse capabilities. Three examples of ex vivo images taken with our system are shown in figure 5.1 .

Figure 5.2 shows chemical selectivity and penetration capabilities of CARS microscopy by imaging a sebaceous gland in a rat ear. The images in this figure are taken by focusing through the skin. The displayed images are the accumulation of 100 images taken at $1-\mu \mathrm{m}$ intervals. The hair can be followed from the skin surface to its root, see figure 5.2(b), and beyond to $100 \mu \mathrm{m}$ depth where the first layer of fat cells can be seen in figure 5.2(c). The gland is composed of individual cells, filled with $\sim 1-\mu \mathrm{m}$ lipid droplets. The cell nuclei appear as darker shadows inside the cells. Deeper penetration depths can potentially be obtained by using adaptive optics [75] to repair the distorted focus in the highly scattering sample.

The CARS signal has a cubic dependence on the intensity and needs two input wavelengths (pump/probe and Stokes) to generate the CARS 

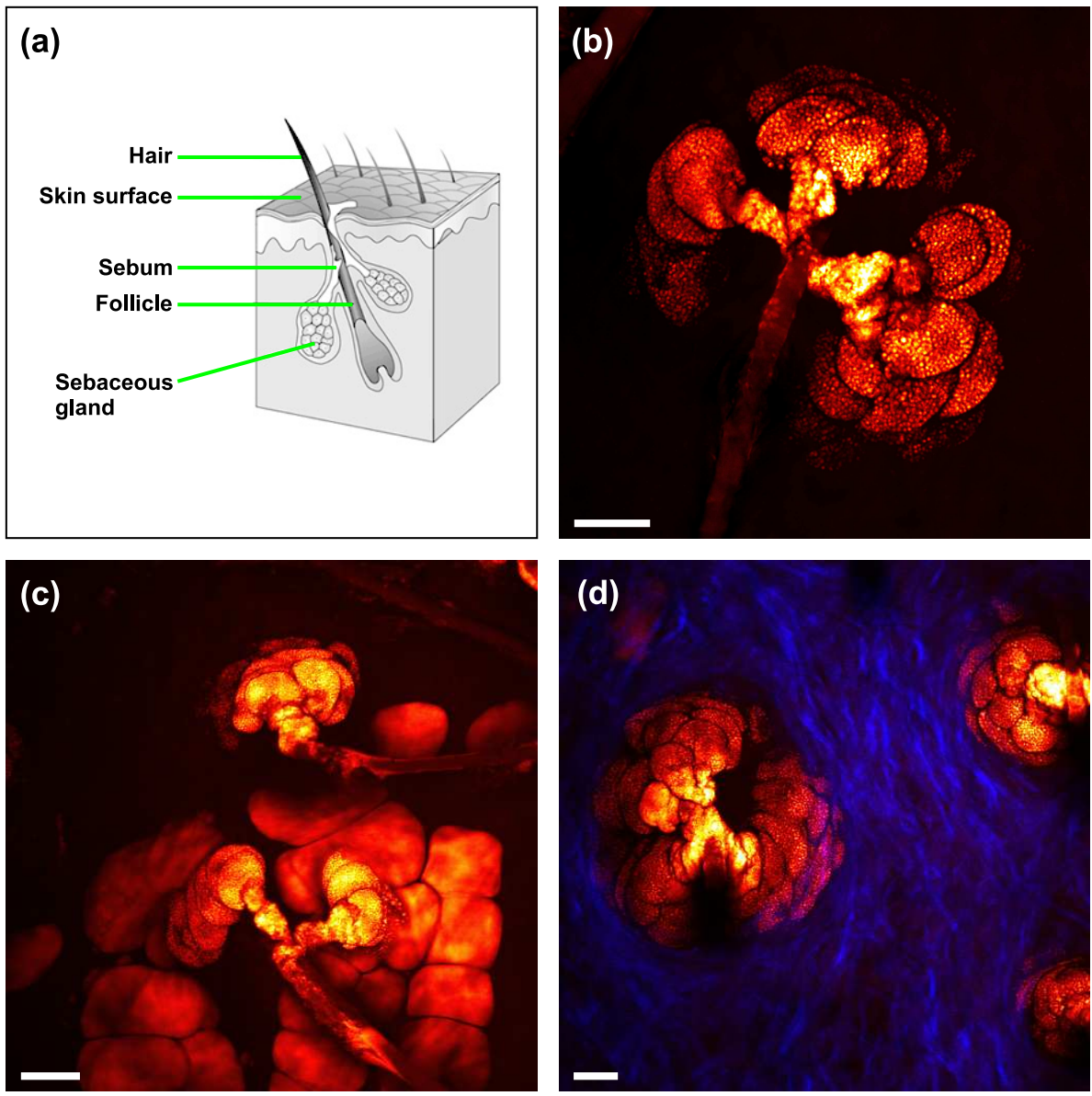

Figure 5.2: Non-invasive imaging of the sebaceous gland in a rat ear. (a) Schematic drawing of a hair in the skin showing the location of the sebaceous gland. (b-c) Image of the hair and sebaceous gland imaged with CARS at $2845 \mathrm{~cm}^{-1}$. (c) additionally shows the first layer of fat cells at a depth of $100 \mu \mathrm{m}$. (d) Image of a combination of CARS and SHG microscopy, where SHG imaging shows the collagen distribution in blue. The scale bar is $20 \mu \mathrm{m}$.

signal. The high intensities and different frequencies in the sample will participate in simultaneous generation of other nonlinear signals. Some of these nonlinear processes are: two photon (auto-) fluorescence (TPF), second harmonic generation (SHG), sum frequency generation (SFG) and third harmonic generation (THG) [76]. In figure 5.3 these nonlinear processes are shown schematically in an energy level diagram. 


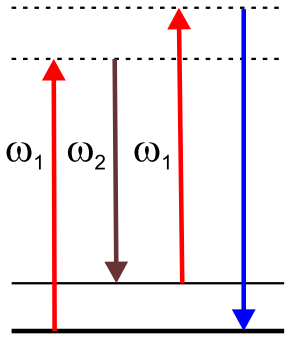

CARS

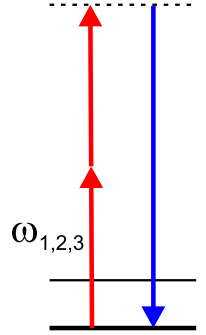

SHG/SFG
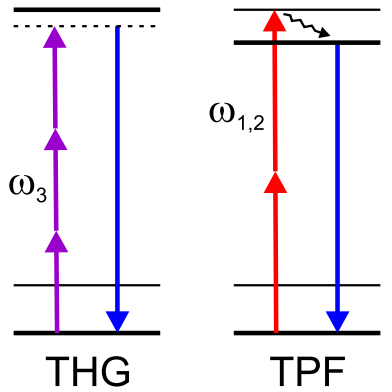

Figure 5.3: Schematic energy level diagram of several nonlinear processes. Solid thick lines represent the electronic ground state and first electronic excited state, solid thin lines represent vibrational levels and dashed lines represent virtual states. $\omega_{1,2,3}$ represent the signal of the $O P O$, fundamental of the laser and idler of the OPO respectively. THG generation with the OPO signal or fundamental frequency are generally to high.

There are several possible combinations of wavelengths, resulting in different detection wavelengths. A proper selection of detector and filters is essential to measure the signal from the nonlinear process of interest and block the remaining signals. For example, using the fundamental of the laser $(1064 \mathrm{~nm})$ and the signal of the OPO $(800 \mathrm{~nm})$ for CARS microscopy will result in a CARS signal at $641 \mathrm{~nm}$. If the sample is also SHG active, SHG/SFG will give signals at 532, 450, and $400 \mathrm{~nm}$. The idler $(1588 \mathrm{~nm})$ of the OPO could furthermore be used for THG, giving a signal at $529 \mathrm{~nm}$.

Second harmonic generation was first demonstrated in 1961 by Franken [77] on a quartz sample. The key requirement for SHG is that the sample material lacks a center of inversion symmetry. Typical noncentrosymmetric structures in nature are starch grains and collagen fibers. In 1986, Freud [78] did the first biological study with SHG microscopy on the orientation of collagen fibers in rat tail tendon. The selectivity of SHG is the result of the structural arrangement at the supramolecular level [18].

Third harmonic generation was first demonstrated in 1967 by New [79] in gases and can be generated in centrosymmetric structures, but is inefficient unless the axial focal symmetry is broken by a change in material properties [80]. An interface breaks the symmetry and creates 
a THG signal. Third harmonic generation is therefore a very sensitive method for the visualization of interfaces in transparent samples and is often enhanced by selecting the input wavelength so that it is close to an electronic resonance. Hemoglobin and lipids are found to provide a resonantly enhanced THG signal, at input wavelengths of $1260 \mathrm{~nm}$ [81] and $1200 \mathrm{~nm}[82]$ respectively.

The advantage of these other nonlinear methods is that they are generated at the same time as the CARS process, but are sensitive to other molecules or structures in the sample. The combination of several nonlinear methods can provide better overview of processes. A tunable picosecond laser system is ideal for CARS microscopy, but gives a lower efficiency of the other nonlinear optical processes [83]. In the setup used in this chapter, THG does not produce enough signal to obtain a useful signal-to-noise ratio and is therefore not used. SHG gives an adequate amount of signal and is used for its selectivity for collagen and non-centrosymetric molecules.

Figures 5.2(d) and 5.4 demonstrate CARS and SHG microscopy. CARS is used for the visualization of the lipid structures and fat cells, and SHG shows the collagen in the tissue. In figure 5.4(b) the SHG reveals a vein embedded between fat cells. The sectioning capabilities of CARS and SHG microscopy allows for three dimensional imaging of the vein. 

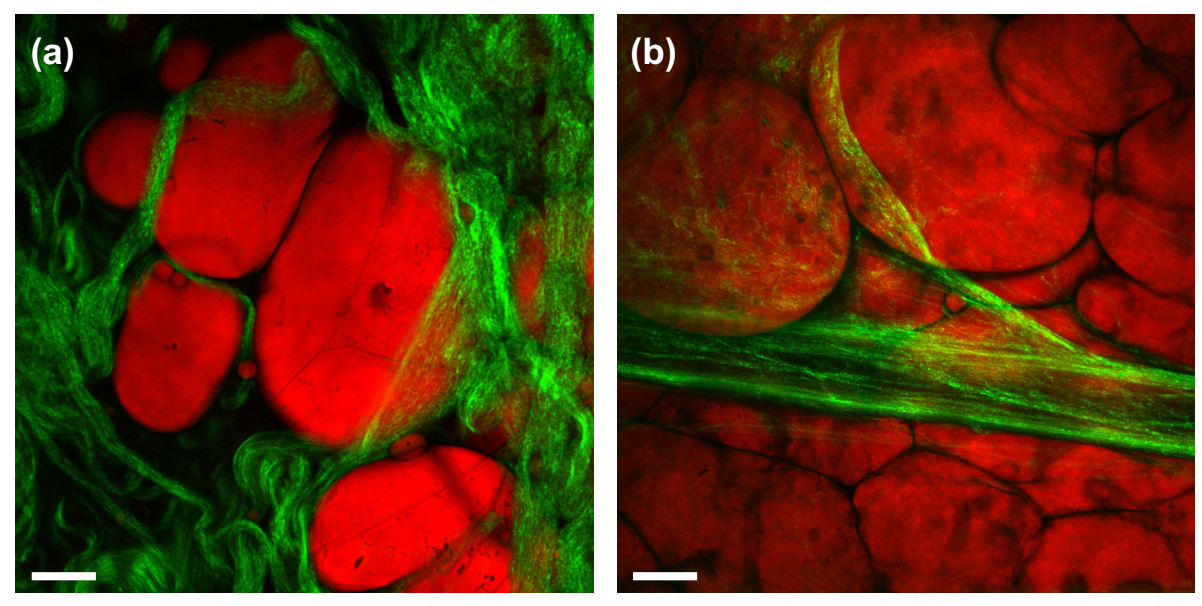

Figure 5.4: (a) Image of beef tissue and (b) an image of a vein in pork tissue. The images show fat cells imaged with CARS microscopy (2845 $\left.\mathrm{cm}^{-1}\right)$ in red and collagen imaged with SHG microscopy in green. The scale bar is $20 \mu \mathrm{m}$. 


\subsection{Chemical imaging and monitoring of phar- maceutical tablets}

\subsubsection{Introduction}

Time- or controlled-release technology in pharmaceutical tablets refers to any mechanism used to release a drug from a tablet slowly and over a pre-determined time window. Figure 5.5 shows a schematic of an instantrelease formulation, where several doses are required over a certain time window, and a controlled-release tablet where one dose is enough to cover the entire window. The advantages of this controlled-release mechanism are that tablets can be taken less frequently and that a more steady drug concentration is maintained in the bloodstream. The first controlled release tablets were made by Howard A. Press in the early 1950s. People diagnosed with angina (severe chest pain) need to take nitroglycerine pills regularly to keep their pain away. Press began developing a method of releasing the nitroglycerine inside the pills over time. The result was a capsule of small drug pellets, where the pellets are coated with different amount of layers which dissolve at different rates. This resulted in a capsule that maintained an effective concentration of nitroglycerine in the body for a longer period of time. This idea of multiple coatings was granted with a US patent in 1960 [84] and became known as controlled release.

Dissolution of drugs from tablets is a complex process that depends on several properties of the drug and the tablet, such as the distribution of the drug in the tablet and the nature and flow of the dissolution medium. One important factor that influences the drug dissolution is the solid state form of the drug. During dissolution, the drug can transform to a less soluble hydrate [85]. For this reason, it is crucial that the solid-state properties are monitored during dissolution. Generally, dissolution testing is a crucial part in pharmaceutical dosage form investigations, performed by analyzing the concentration of the released drug in a defined volume of flowing dissolution medium.

In this study coherent anti-Stokes Raman scattering (CARS) microscopy is used to visualize the release of a drug (theophylline) from a lipidbased (tripalmitin) tablet during dissolution. The molecular structures of these compounds are shown in figure 5.6. With CARS microscopy it is possible to achieve temporally- and spatially-resolved visualization $[86,87]$ of the distribution and the solid-state properties of the powders 


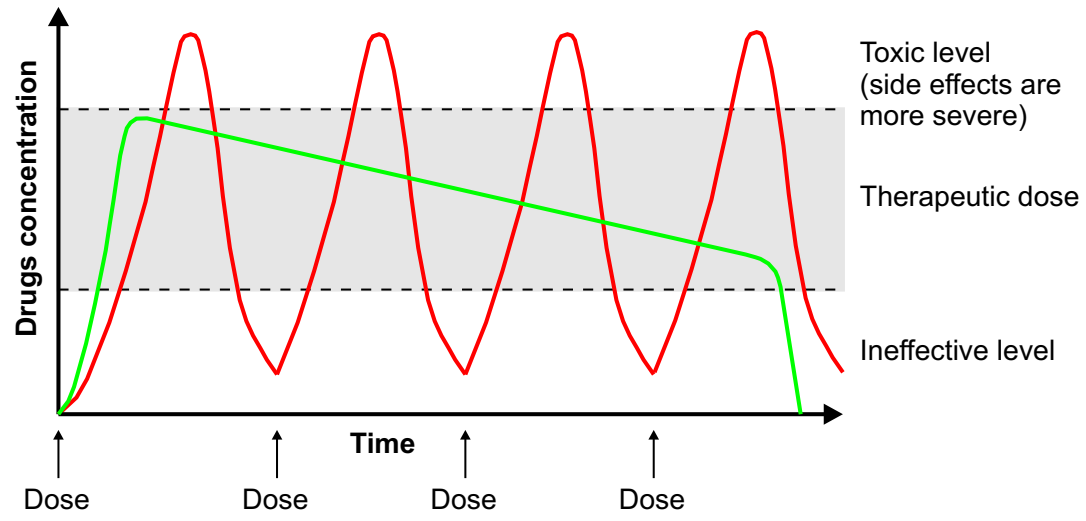

Figure 5.5: Schematic of controlled released (green) and conventional drug release (red). For the conventional drug release the time of each dose is shown and the overshoot (above toxic level) and undershoot (ineffective level) are shown. Where the therapeutic dose is covered by the time released tablet during the complete time.

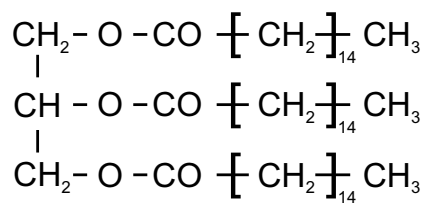

Tripalmitin<smiles>Cn1c(=O)c2[nH]cnc2n(C)c1=O</smiles>

Theophylline

Figure 5.6: Molecular structure formula of the tripalmitin lipid and theophylline drug

and tablets. The results obtained by CARS microscopy reveal that the method used to combine the lipid and the active ingredient (drugs) into the tablet can influence the physicochemical behavior of the drug during dissolution. The manufacturing process causes significant differences in the release process: tablets prepared from powder show formation of theophylline monohydrate on the surface which prevents a controlled drug release, whereas solid lipid extrudates do not show formation of monohydrate. The use of CARS microscopy in pharmaceutical dosage form investigation gives new insight in future tablet design. 


\subsubsection{Conventional techniques}

Important properties of the solid dosage form include the apparent solubility of the drug and the other components, particle size, and drug distribution. These characteristics, and hence the dissolution rate, change during drug dissolution [85]. Dissolution testing is universally used in the development, production, and quality assurance of oral solid dosage forms. During such testing, the dosage form is immersed in a flowing aqueous medium and the concentration of the released drug in the medium is measured at defined time intervals using techniques such as UV spectroscopy or high performance liquid chromatography (HPLC). Although valuable, such analysis provides no direct information on the changing dosage form phenomena, and hence the dissolution behavior of drugs cannot be completely understood with these techniques alone. Hence, there is a need to monitor the changing chemical and physical properties of dosage forms during dissolution. Chemical selectivity, good spatial resolution and fast imaging for in situ analysis are needed, which CARS microscopy provides. The CARS technique has recently been used to monitor drug distribution and release from polymer films used in stents [86]. In a subsequent study, drug crystallization during preparation of films was observed for stent coating materials [88]. To the best of our knowledge, oral dosage forms and their physicochemical changes during dissolution have not previously been visualized with the use of CARS microscopy.

\subsubsection{Experimental system}

Lipid-based formulations have shown promise as controlled release oral dosage forms as they can effectively control the release rate of drugs, they are physiological and therefore nontoxic, they mask the unpleasant taste of drugs, and no organic solvents are required during dosage form preparation. In these studies different lipid-based oral dosage forms are investigated. Physical powder mixtures of lipid and drug were compressed and compared to extruded matrixes consisting of the same components. In addition, the extruded matrixes were compressed to tablets. The aim of the study was to combine CARS microscopy with a suitable flow-through cell setup as a means to gain deeper understanding of the physicochemical behavior of oral dosage forms during dissolution.

In this study of dissolution, two different types of pharmaceutical tablets and three model systems of a drug (theophylline) in a matrix 
(tripalmitin) are investigated:

- tablets of tripalmitin with theophylline monohydrate,

- tablets of tripalmitin with theophylline anhydrate,

- extrudate of tripalmitin with theophylline anhydrate,

- tablets from an extrudate of tripalmitin with theophylline anhydrate.

The tablets are made by compressing the powder mixture in a tableting machine at room temperature. The extrudates are made in a corotating twin-screw extruder at a processing temperature of $55{ }^{\circ} \mathrm{C}$. More details of the sample preparation are given in [89].

The CARS setup is based on a Coherent Paladin $\mathrm{Nd}: \mathrm{YVO}_{4}$ laser and an APE Levante Emerald Optical Parametric Oscillator (OPO). The fundamental $(1064 \mathrm{~nm})$ of the laser is used as Stokes, whereas the signal from the OPO (tunable between 700-1000 nm) is used as the pump and probe. The beams are scanned over the sample by galvano mirrors (Olympus FluoView 300, IX71) and focused by a 20x 0.5NA objective lens into the sample. Both beams have a power of several tens of $\mathrm{mWs}$ at the sample. Due to the highly scattering samples the forward generated CARS signal is scattered and part of it is collected in the backward direction. The collected signal is filtered and detected by a photo multiplier tube (Hamamatsu R3896). All images are 512x512 pixels and are obtained in 2 seconds. Images at different wavelengths require tuning of the OPO, but no realignment of the optics. For the dissolution testing, the tripalmitin matrix was imaged before and after dissolution to verify the absence of change in the matrix material. During the dissolution, only the theophylline was imaged in real time.

To determine suitable vibrational bands for component-specific imaging and analysis, Raman spectra were recorded of pure tripalmitin, theophylline anhydrate, theophylline monohydrate and water. Spectra were taken using a 1600 pixels CCD camera (Newton DU-970N, Andor Technology). The samples were irradiated by a Kr-ion Laser (Coherent, Innova $90 \mathrm{~K}$ ) of $30 \mathrm{~mW}$ (at $647.1 \mathrm{~nm}$ ) and the light was focused by $40 \mathrm{x}$ 0.65NA microscope objective lens. Care was taken to ensure that the focal spot was filled with pure material. Figure 5.7(a) shows the Raman spectra of the three powdered substances. The high frequency region is highlighted in gray and shown with the main vibrational stretches in 

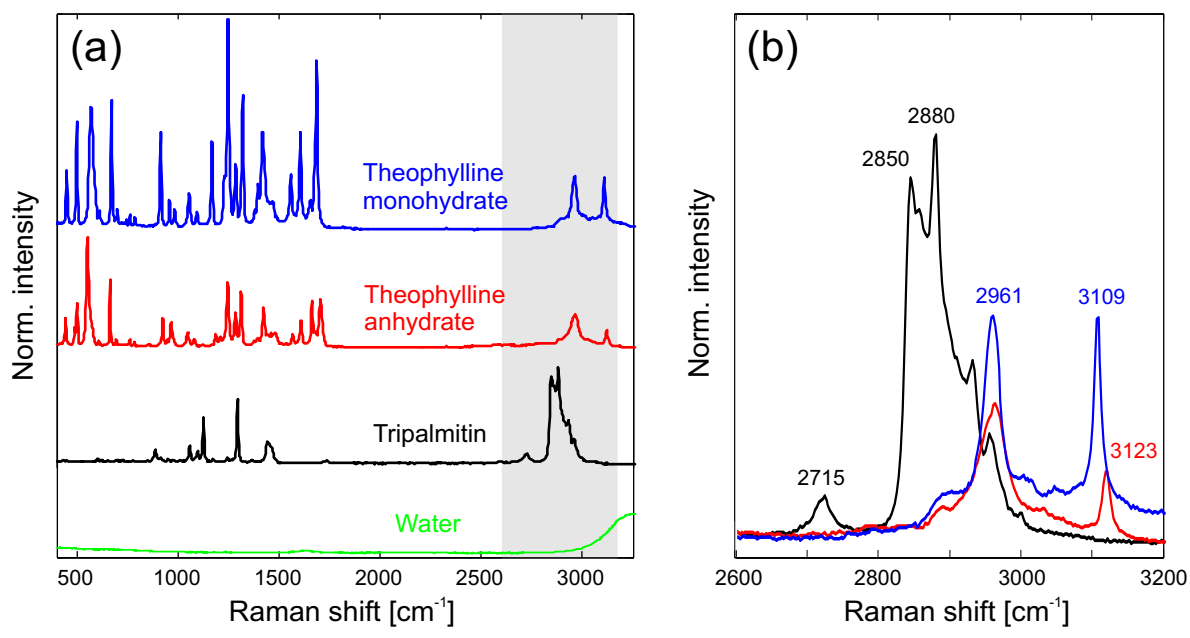

Figure 5.7: Raman spectra of the powdered substances and water: (a) full spectra of tripalmitin (black), theophylline anhydrate (red), theophylline monohydrate (blue) and water (green) and (b) highlighted detail of image (a).

figure 5.7(b). Between 2700 and $3200 \mathrm{~cm}^{-1}$, the $\mathrm{CH}$ stretch in the aldehyde function of tripalmitin gives rise to a peak at $2715 \mathrm{~cm}^{-1}$ whereas the $\mathrm{CH}_{2}$ and $\mathrm{CH}_{3}$ symmetric stretching in the fatty acid chains correspond to peaks at $2850 \mathrm{~cm}^{-1}$ and $2880 \mathrm{~cm}^{-1}$, respectively [90]. Theophylline exhibits $\mathrm{CH}_{3}$ antisymmetric stretching at $3109 \mathrm{~cm}^{-1}$ for the theophylline monohydrate and $3123 \mathrm{~cm}^{-1}$ for the anhydrate [91, 92]. Specific component analysis in the 1600 to $1800 \mathrm{~cm}^{-1}$ region is not favorable due to the water vibrational modes around $1650 \mathrm{~cm}^{-1}$ and is therefor not used for real time dissolution experiments. In the 2700 to $3200 \mathrm{~cm}^{-1}$ region tripalmitin and theophylline can be imaged selectively using the peaks at $2880 \mathrm{~cm}^{-1}$ (tripalmitin) and $3109 \mathrm{~cm}^{-1}$ (theophylline). Unfortunately, a distinction between theophylline anhydrate and monohydrate is not possible in this region. Nevertheless, the peak at $3109 \mathrm{~cm}^{-1}$ can be used to selectively image both forms of theophylline. The interference from water in this region would seem large as well, but these bands are so broad and dephase so quickly that their influence is limited.

Dissolution testing is performed with a flow-through cell. The flow cell used for the in situ dissolution imaging consists of a Teflon chamber with two metal bars that fix the tablets in the middle of a flowing water bed (see figure 5.8). A hose pump circulates purified water (at room 


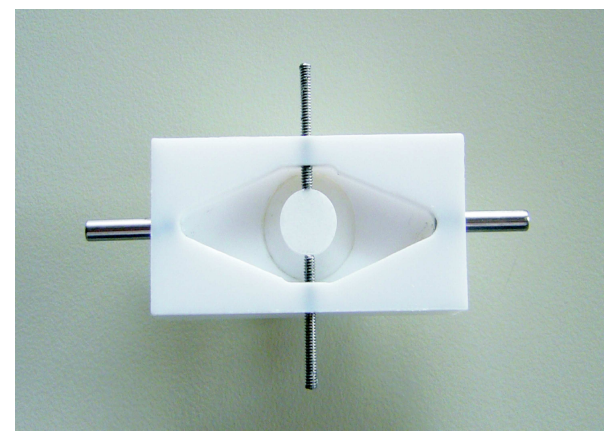

Figure 5.8: Photograph of the flow cell with tablet (without the cover glass)

temperature) through the chamber via suitable conduits at a constant rate of $5 \mathrm{~mL} / \mathrm{min}$. The lower side of the cell consists of a thin microscope cover glass that is placed on the microscope stage. This specific set-up allows in situ visualization of the solid-state properties of the tablets under pharmaceutically relevant dissolution conditions [93].

\subsubsection{Dissolution experiments}

Tablets and extrudates were analyzed using CARS microscopy to visualize the distribution of lipid and drug in the dosage forms. Figure 5.9 shows false-color images of the surface of the tablet and extrudate model systems. The drug is always depicted in green, irrespective of its solid state form. The lipid matrix is depicted in red. A mix of both signals appears yellow. Since yellow is not observed, the substances do not mix at this resolution $(\sim 1.5 \mu \mathrm{m})$.

The first column depicts the three dry tablet and the extrudate system. The surface of the theophylline monohydrate (figure 5.9(a)) is characterized by thin needles whereas the anhydrate (figure 5.9(d)) shows anisometric particles. The tablet of compressed extrudates (figure $5.9(\mathrm{~g})$ ) shows a smaller portion of drug signal on the surface, so that the drug can be considered more embedded in the matrix or may be attributed to a depletion of drug adjacent to the extruder barrel wall during processing [94], not observed on an uncompressed extrudate, figure $5.9(\mathrm{j})$. On the dry tablet the surface coverage was determined as

- figure 5.9(a) tablet tripalmitin/theophylline monohydrate (40/60\%), 
- figure 5.9(d) tablet tripalmitin/theophylline anhydrate (60/40\%), and

- figure $5.9(\mathrm{~g})$ tablet of extrudates tripalmitin/theophylline anhydrate $(80 / 20 \%)$.

The lipid-based dosage forms were imaged after immersion in 500 $\mathrm{mL}$ of purified water and compared with the images of the dosage forms before dissolution. After 30 and $180 \mathrm{~min}$ of immersion, they were removed and imaged using CARS microscopy to investigate drug release and solid-state transformations. The release of the drug from a tablet consisting of a physical mixture of tripalmitin and theophylline monohydrate (figure 5.9(a-c)) is evident from a gradual loss of green color representing the drug. After 30 min of immersion, the drug was still visible whereas after $180 \mathrm{~min}$ no drug was evident on the surface of the tablet. Instead, pores in the lipid matrix where drug needles were located are represented as dark areas in the false-color image. The lipid (red color) remained after dissolution, demonstrating that the matrix stays completely intact during dissolution. It can thus be concluded that the release of the drug is purely diffusion controlled with the drug initially dissolving at the surface of the matrix and then dissolving within the matrix and diffusing thorough the resulting pores in the matrix.

Different phenomena were observed in the tablet consisting of tripalmitin and theophylline anhydrate (figure 5.9(d-f)). Figure 5.9(d) depicts the tablet surface before immersion with the anhydrous theophylline clearly visible as dispersed particles in the lipid matrix. After 30 min of immersion in water, the surface of the tablet was covered by fine needles could be observed by the green false color (figure 5.9(e)). This can be attributed to the solution-mediated formation of theophylline monohydrate, a phenomenon which has previously been observed upon immersion of the anhydrate in water. In these studies [95, 96, 97], monohydrate growth in water has always been associated with needlelike morphology. After 180 min of immersion in water, needles (green) completely disappeared and the lipid (red) was once more visible, suggesting that the theophylline monohydrate had completely dissolved.

The dissolution process of theophylline anhydrate in this dosage form can therefore be subdivided in several stages. First, the theophylline anhydrate dissolves and, with the monohydrate being less soluble than the anhydrate, a supersaturated solution with respect to theophylline monohydrate is created. This is followed by a transformation phase in 

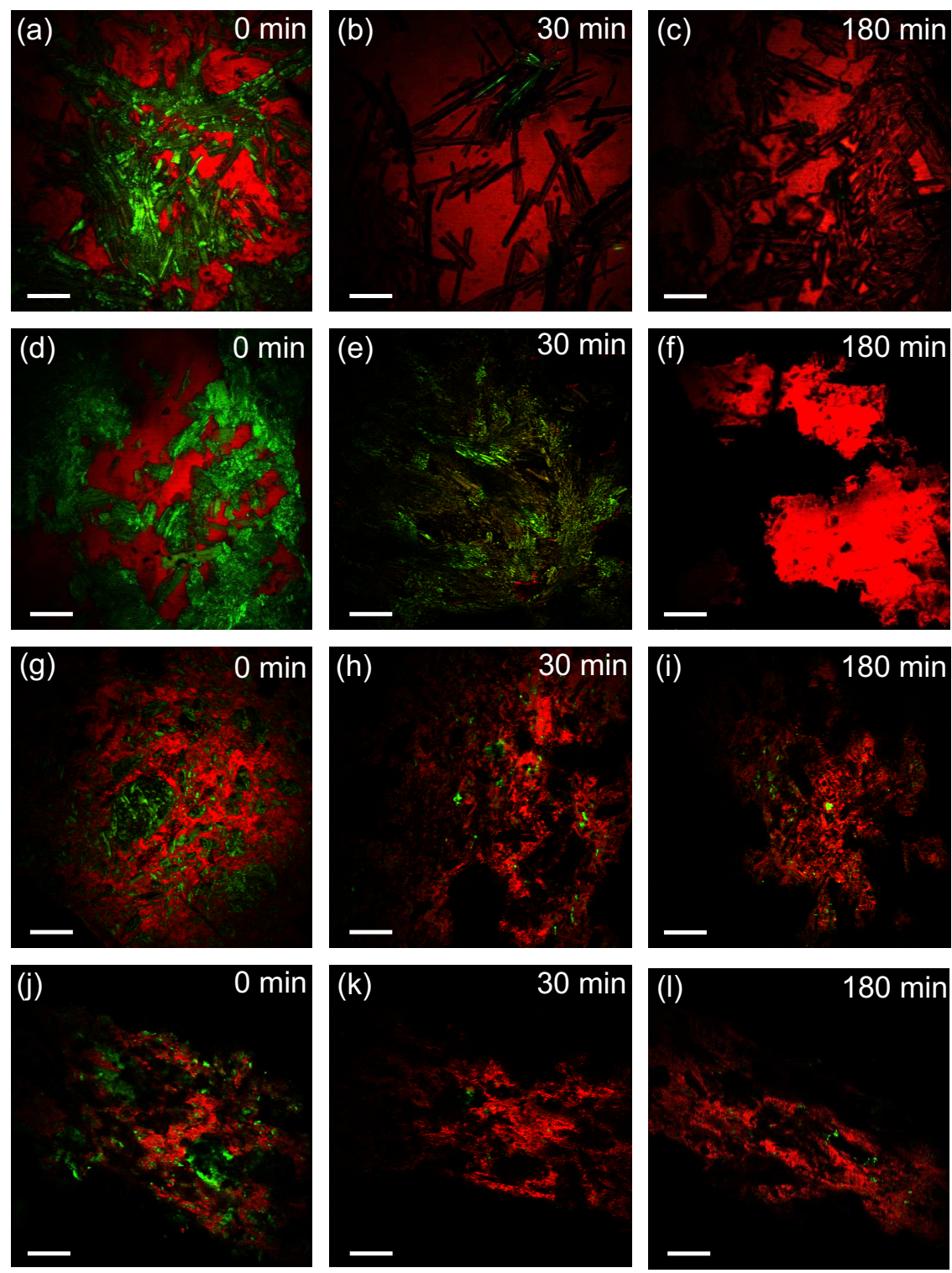

Figure 5.9: Images of the lipid tripalmitin (2880 $\mathrm{cm}^{-1}$, red) and the drug theophylline (3109 $\mathrm{cm}^{-1}$, green) after different durations of immersion. (a-c) tablet tripalmitin/theophylline monohydrate (d-f) tablet tripamitin/theophylline anhydrate ( $g$-i) tablet of extrudates tripalmitin/theophylline anhydrate ( $j-l)$ extrudate of tripalmitin/theophylline anhydrate. The scale bar is $100 \mu \mathrm{m}$. 
which the monohydrate crystallizes [98]. Afterward, dissolution of the two forms takes place. To investigate the influence of the extrusion process on the release behavior of the drug, a tablet was compressed from tripalmitin and theophylline anhydrate extrudates and subjected to the same dissolution study. For this tablet (figure 5.9(g-i)), release of the anhydrate particles was observed but, strikingly, no monohydrate needle formation on the tablet surface was observed.

With the CARS microscopy setup, additional studies were conducted using the uncompressed extrudates made of tripalmitin and theophylline anhydrate (results depicted in figure $5.9(\mathrm{j}-\mathrm{l})$ ) and in these unmodified extrudate samples there was also no evidence of theophylline monohydrate formation during the dissolution of the anhydrate particles. A scan in the pores of the extrudate at a depth of $50 \mu \mathrm{m}$ was performed to observe physicochemical changes within the matrix in addition to at the surface. At this depth a few very small monohydrate needles can be found inside the pores. Further investigation is required to understand the reason for such different solid-state behavior of the unextruded and extruded samples. However, in an attempt to understand such behavior, one can look at the mechanism of crystallization of the monohydrate. The mechanism of conversion is believed to be solution-mediated. First, nucleation from solution must occur followed by crystal growth. Nucleation typically occurs in the presence of a suitable surface and supersaturated solution with respect to the monohydrate, and crystal growth is dictated by the degree of supersaturation. The outer surface of the extrudate may be a poor substrate for nucleation since it is very smooth, created by the temperature of the extrusion process. In contrast, the surface of the compressed powder mixtures is likely to be rougher. With regard to supersaturation, as already stated it appears that the extrusion process reduces the drug exposure at the surface of the extrudate and hence of the surface of the tablet consisting of extrudates, which may mean that the solution adjacent to the surface is less supersaturated with respect to the monohydrate inhibiting its nucleation and crystal growth. Within the pores, the rougher surface which is left after the release of drug particles in the pores may promote nucleation. In addition, the diffusion within the small pores is likely to be very slow, and therefore through greater supersaturation in these regions crystal formation is more likely to occur.

To monitor the solid-state transformation in real time, tablets consisting of a mixture of tripalmitin and theophylline anhydrate were placed 

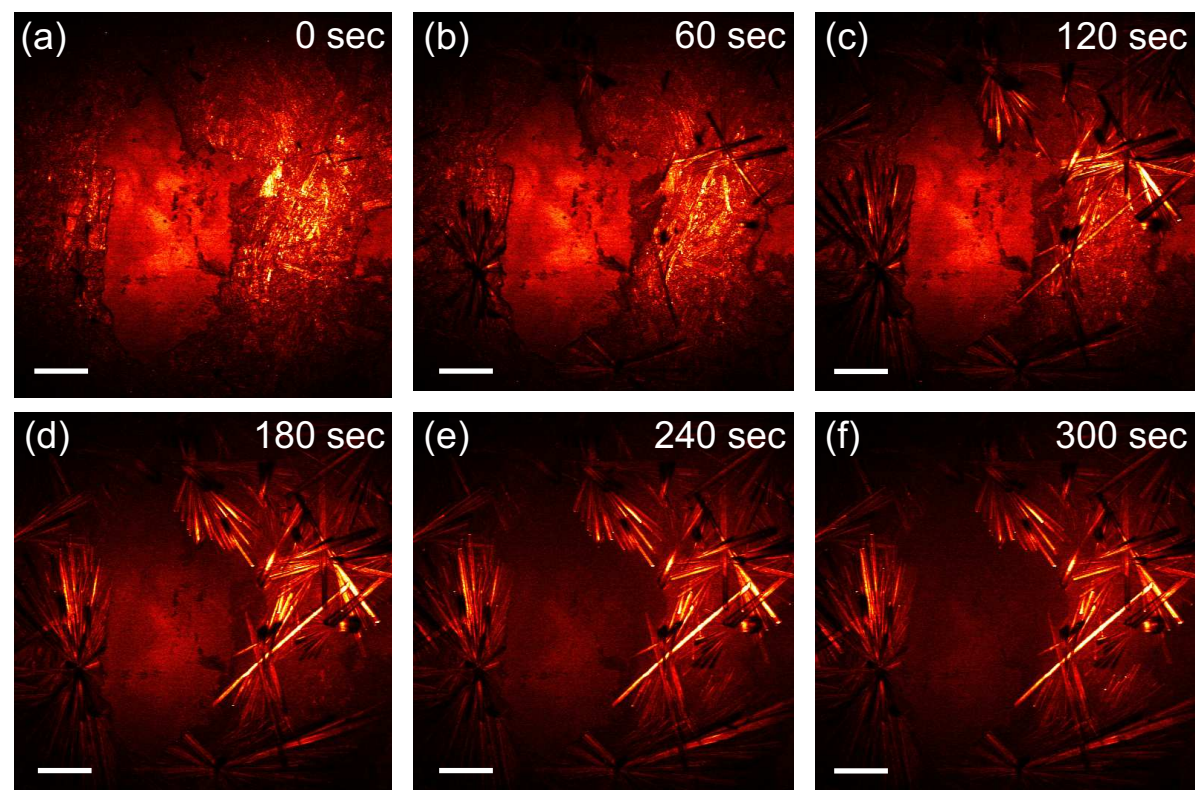

Figure 5.10: Inline visualization of theophylline monohydrate crystal growth on the surface of a tablet consisting originally of tripalmitin/theophylline anhydrate in water. The scale bar is $100 \mu \mathrm{m}$.

directly on the microscope stage in a small container mounted on a thin glass slide which was filled with purified water so that a thin water layer was located between the sample and the microscope objective. Figure 5.10(a-f) depicts several frames of the recorded images. In real time, the transformation from theophylline anhydrate to monohydrate could be visualized for the first time on a tablet surface. When a thin stagnant water layer between the tablet and the microscope cover slide is created, growth of extra long needles is promoted resulting in images such as figure 5.11 .

For dissolution testing, tablets containing monohydrate were continuously imaged over 90 minutes while placed in the flow cell (see figure 5.12), which provides a pharmaceutically relevant dissolution setup for oral dosage forms. Focal drift and stability issues prevented a direct quantitative analysis of the images, but controlled release of the drug was clearly observed and quantitative analysis should be possible once the stability is improved. 


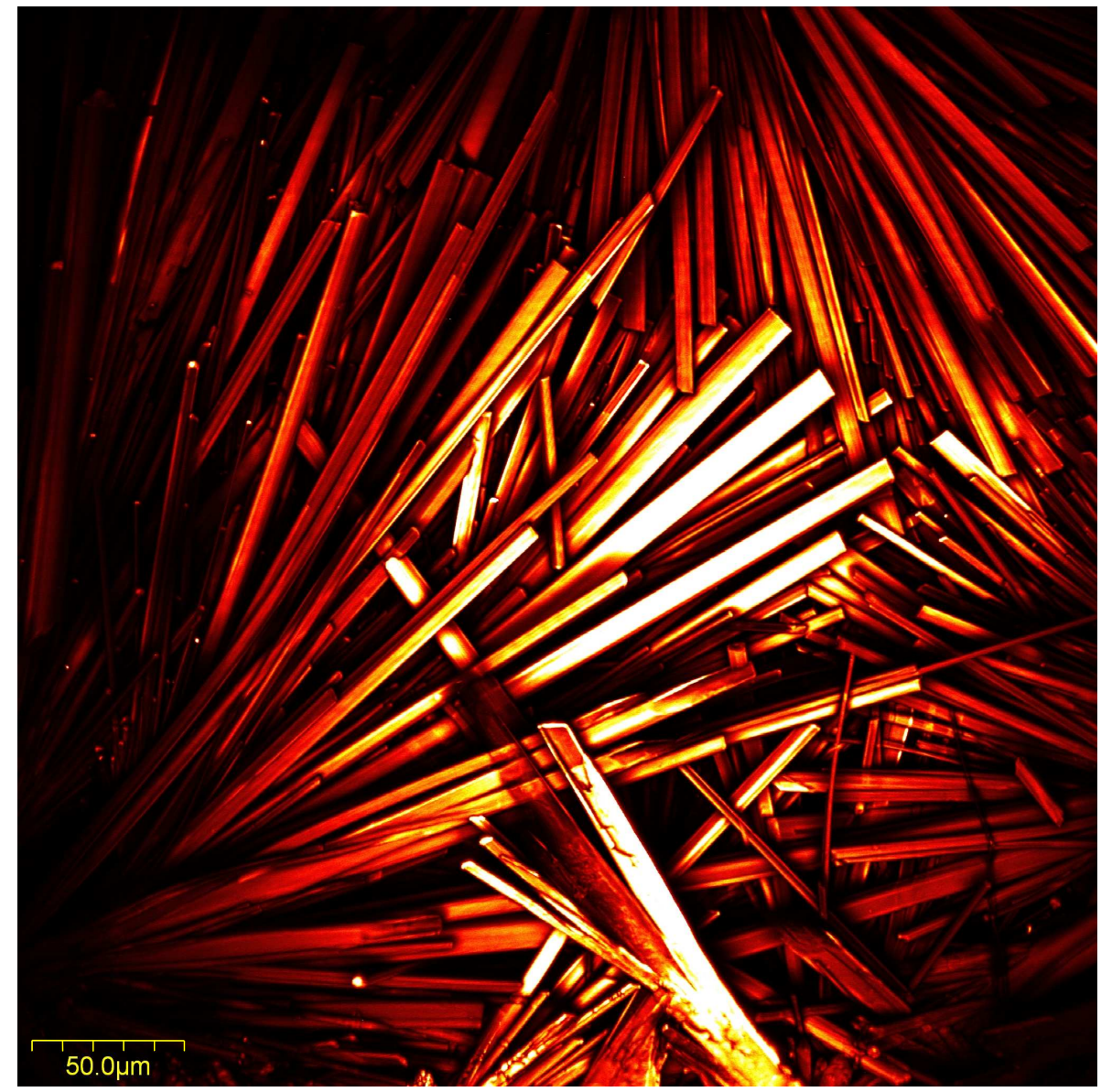

Figure 5.11: Formation of long theophylline monohydrate needles by using a thin stagnant water layer between the theophylline anhydrate tablet and the microscope coverslide.

\subsubsection{Conclusion and outlook}

CARS microscopy was used to visualize the spatial distribution of different components in oral pharmaceutical dosage forms and, by combining the method with a suitable flow-through cell, drug release and physicochemical changes during dissolution testing were monitored in real time. As solid-state properties of pharmaceutical dosage forms affect the dissolution behavior, the visualization of solid-state changes with this method can expand the knowledge about dissolution mechanisms. Such knowledge will help lead to tailor-made dissolution profiles by manufacturing of pharmaceutical dosage forms that exhibit desirable 

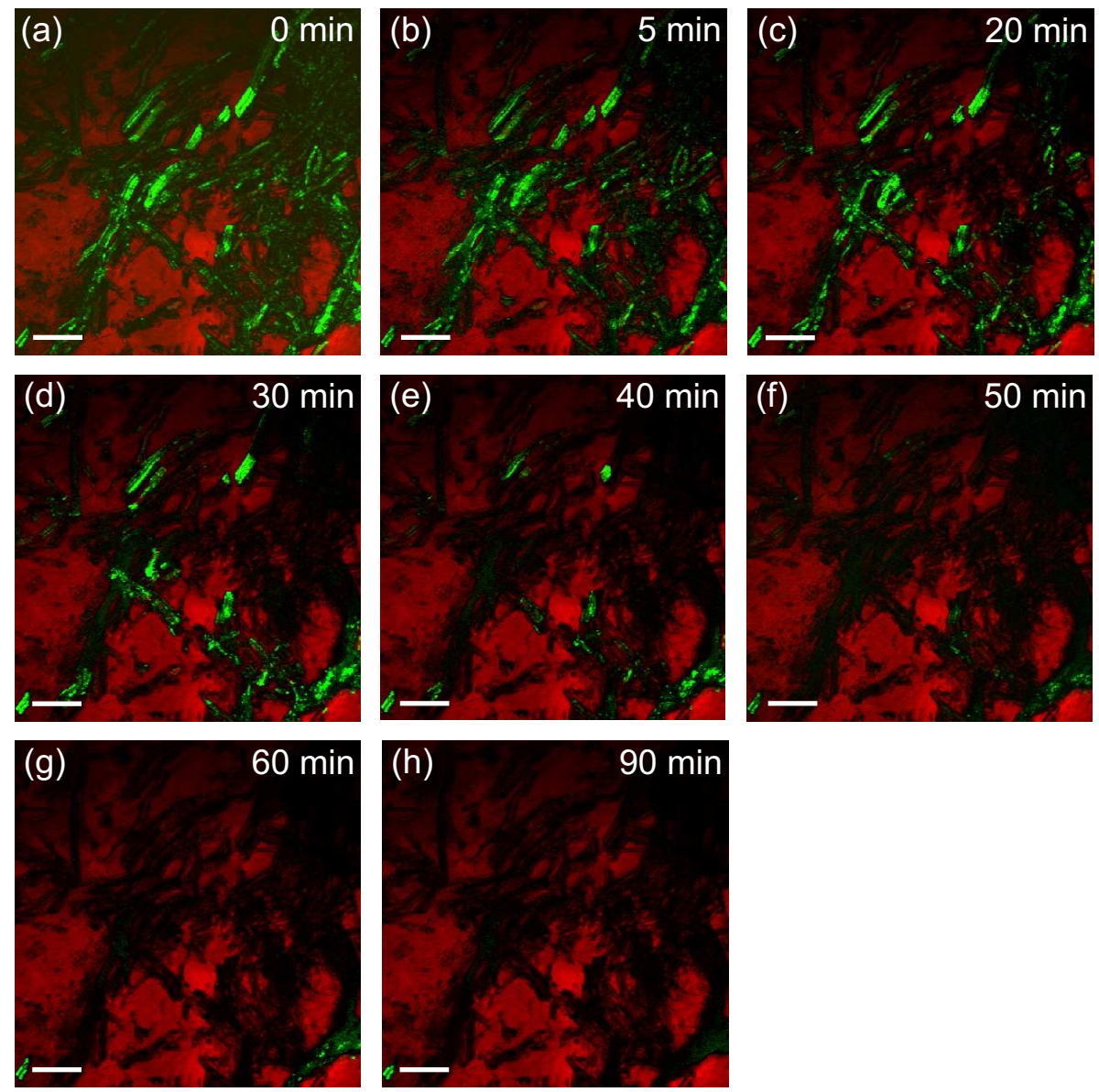

Figure 5.12: In situ CARS imaging of drug release during dissolution in the flow cell. The lipid tripalmitin $\left(2880 \mathrm{~cm}^{-1}\right.$, red) and drug theophylline monohydrate (3109 $\mathrm{cm}^{-1}$, green) are shown. The scale bar is $100 \mu \mathrm{m}$.

physicochemical properties during dissolution. In the case of solid lipid extrudates, the drug and lipid distribution on the surface of the solid dosage form was rapidly visualized with a spatial resolution of $\sim 1.5 \mu \mathrm{m}$ and a frame every 2 seconds with CARS microscopy. Furthermore CARS microscopy was used to monitor the loss of the model drug theophylline from the lipid matrix as well as solution-mediated solid-state transformations (from theophylline anhydrate to the monohydrate form) on the surface of tablets in real time. Solid lipid extrusion prevented theophylline hydrate formation, which is probably due to surface differences of 

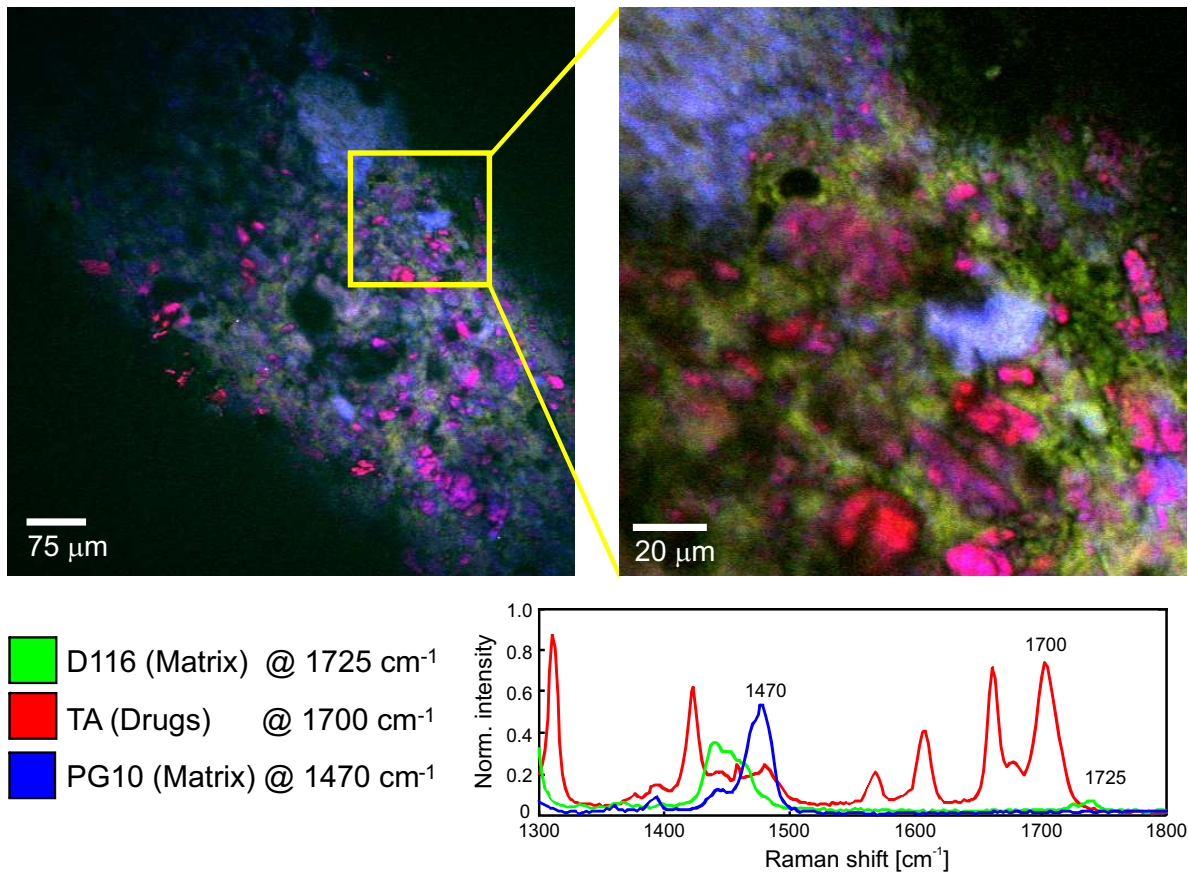

Figure 5.13: Dry CARS image and contributed Raman spectrum of polyethylene glycol (PG10, 25\%, matrix), trimalmitin (D116, 25\%, matrix) and theophylline anhydrate (TA, 50\%, drugs) of extrudate

the lipid matrix. On the basis of these results, CARS microscopy may be a valuable characterization method in the future development of different kinds of oral solid dosage forms.

Depending on the type of drugs in a tablet, the tablet material may be suitable for controlled release or not. More matrix materials have to be studied as well as combinations of matrix materials like lipids and gels to obtain more variable parameters to obtain the ideal dissolution profile [99]. As a preliminary experiment a three compound dry tablet is imaged, containing theophylline anhydrate (drugs), trimalmitin (lipid matrix) and polyethylene glycol (gel matrix), see figure 5.13. 


\subsection{How cells grow bone}

\subsubsection{Introduction}

The growth of bone starts at the cellular level, where the progression through different stages in the formation process of new bone is called the osteogenic lineage, shown in figure 5.14. It starts with stem cells, which have the ability of self-renewal, but once differentiated the cell becomes lineage-restricted (multipotent). The stem cells in this study are retrieved from adults. The mesenchymal stem cell can differentiate into several different lineages, three of them are:

- Osteoblasts - lead to bone formation.

- Chondrocytes - lead to cartilage formation.

- Adipocytes - also known as lipocytes or fat cells, lead to storage into the form of fat.

By specific growth factors, the differentiation of the mesenchymal stem cells towards osteoblast cells can be controlled as described by Pittenger et al. [100]. The osteogenic differentiation process follows several steps, starting with the differentiation towards osteoprogenitor. These cells allow for proliferation, cell development and cell division. Further differentiation results in preosteoblast cells, which are responsible for the syntheses of the cell matrix structure. In the next step the cell becomes a mature osteoblast and when the osteoblast becomes trapped in the matrix structure, it becomes an osteocyte. From this moment on hydroxyapatite that contains phosphate and carbonate ions is deposited in the matrix, leading to mineralized extra cellular matrix that is analogous to in vivo bone [101].

In this study multipotent adult human bone marrow stromal cells are visualized at specific points in a 60-day culture period. The observations cover formation processes of bone at five-day intervals from day 25 until day 60. A combination of Raman spectroscopy, CARS microscopy and second harmonic generation (SHG) microscopy is used. Based on the results a model for bone growth is presented. The model contains the following steps:

- The proliferation of the cells, the growth of lipid droplets and synthesis of collagen.

- Mineralization around lipid droplets. 


\section{Osteogenic lineage}

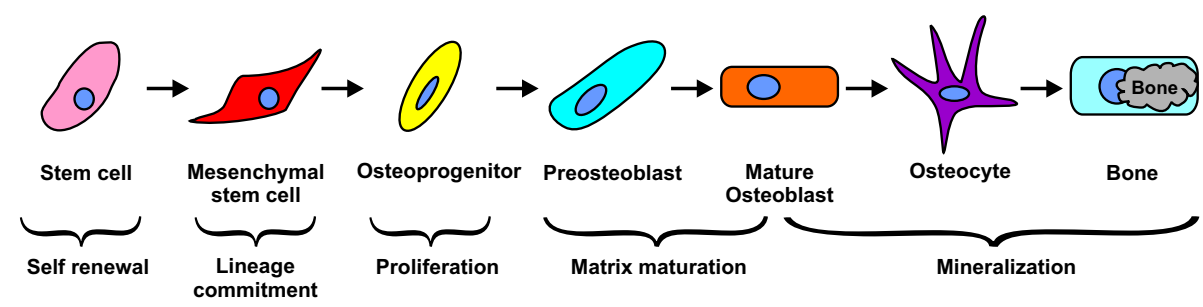

Figure 5.14: Schematic overview of the osteogenic lineage, showing the differentiation steps from stem cell to bone formation [102].

- Collagen fibrils guiding the bone layer growth.

- Three dimensional bone formation.

\subsubsection{Previous work}

The in vitro differentiation of multipotent human mesenchymal stromal cells towards bone has been explored previously [100, 103, 104]. There are two different hypotheses for the start of the mineralization process of hydroxyapatite in vivo.

- Hypothesis 1: The cells construct collagen fibrils (type-I) with holes and pores in the interfibrilar spaces. These holes and pores are filled with several types of non-collagenous proteins which are unique to bone and help in mineralization [102].

- Hypothesis 2: There is formation of matrix vesicles $(0.1-1 \mu \mathrm{m})$ which are cell-derived extracellular membrane-enclosed particles [105]. These matrix vesicles are composed of calcium ions (binding phosphates), proteins and small amounts of collagen (type-II) and enable the initial mineralization [106, 107, 108].

Furthermore it has recently been demonstrated that cholesterol - which plays an important role in the differentiation towards osteoblasts [109, 110 - also plays an active role in the hydroxyapatite mineralization $[111,112]$.

A recent study, performed with Raman spectroscopy [113], showed that the formation of mineralized nodules in the cells is influenced by the presence of phospholipids in the lipid droplets in the cells. Studies 
on chicken embryo osteoblasts with different visualization techniques demonstrated the growth of three to four layers of cells. Each layer of cells showed an assembly of collagen fibrils. Within these studies, the assembly of collagen fibrils was shown to play a role in the mineralization process [114]. Furthermore, the formation of calcium phosphate towards carbonated calcium phosphate by osteoblast cells has been observed [115]. There are also observations of lipid droplets under the mineralized collagen layer, indicating their role in the calcification of collagen in the formation of compact bone [116].

In this study the differentiation and mineralization of multipotent adult human bone marrow stromal cells is monitored by Raman spectroscopy, CARS and SHG microscopy.

\subsubsection{Experimental setup}

The cells are retrieved from healthy donors and seeded on multiple glass cover slips at a cell density of 1000 cells $/ \mathrm{cm}^{2}$. Cell measurements are performed on every fifth day from day 25 until day 60 . The Raman spectroscopy measurements are performed on living cells, whereas the CARS and SHG measurements are performed after fixing the cells with $1 \%$ paraformaldehyde.

A confocal Raman microspectroscope [117] is used to perform measurements on the living cells. $35 \mathrm{~mW}$ of power from a Krypton ion laser (Coherent, Innova 90K) emitting at $647.1 \mathrm{~nm}$ is used as excitation source. A $63 x 1.2$ NA water immersion objective (Zeiss Plan Neofluar) is used to focus the laser light on the sample and collect the Raman scattered photons from the sample. The scattered photons are focused on a confocal pinhole placed in front of a spectrograph and detected by an air-cooled CCD camera (Newton DU-970N, Andor Technology). This combination provides a spectral resolution of 1.85 to $2.85 \mathrm{~cm}^{-1} /$ pixel over a Raman shift range of -20 to $3670 \mathrm{~cm}^{-1}$. Spectra are recorded in $100 \mathrm{~ms}$.

The CARS microscopy setup with SHG imaging capability starts with a Coherent Paladin Nd: $\mathrm{YVO}_{4}$ laser and an APE Levante Emerald OPO. The OPO signal (tunable between 700-1000 nm) is used as pump and probe wavelength while the fundamental $(1064 \mathrm{~nm})$ wavelength from the laser is used as Stokes wavelength in the CARS process. Both beams 

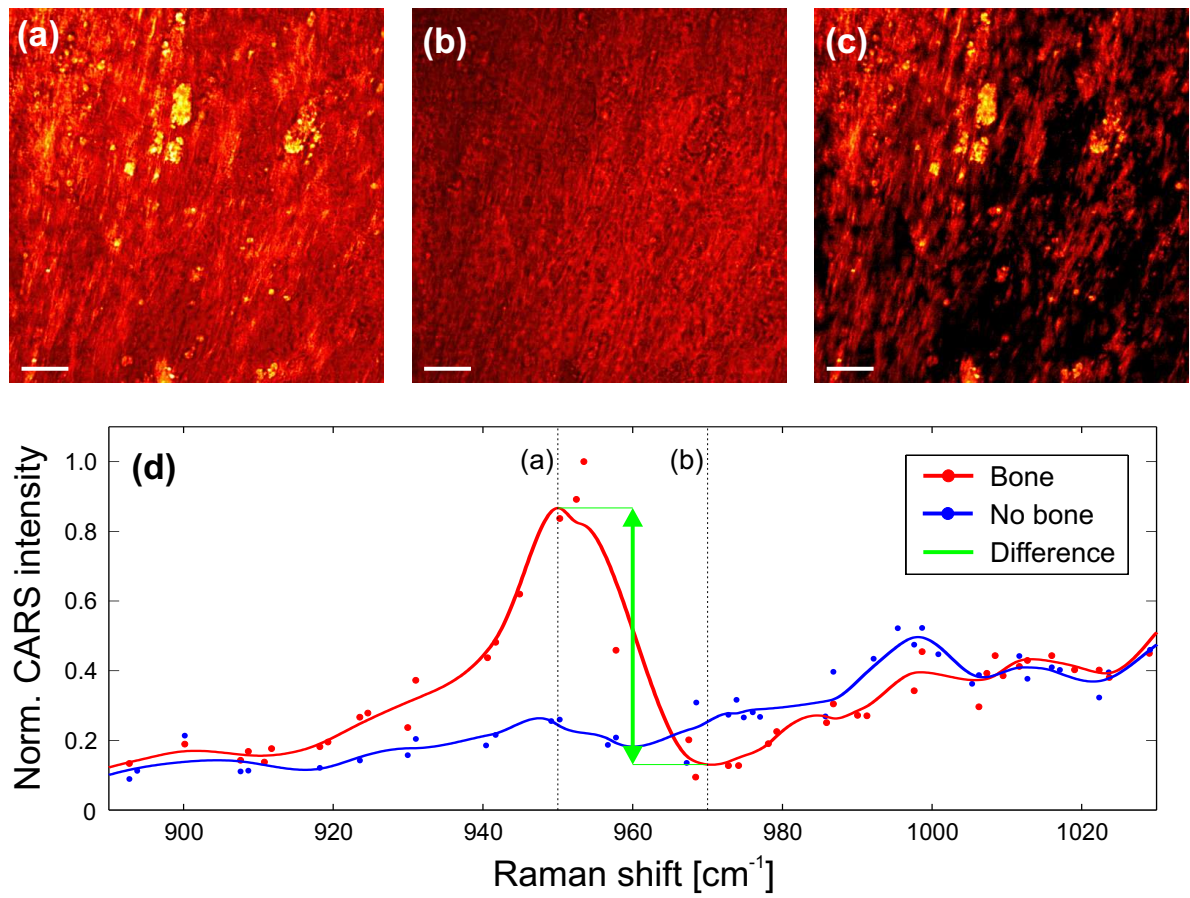

Figure 5.15: (a) and (b) show images at $950 \mathrm{~cm}^{-1}$ and $970 \mathrm{~cm}^{-1}$ respectively. The subtraction results in an image of only the resonant bone (c). The spectra of the bone and the non-resonant background are shown in (d). The scale bar is $20 \mu \mathrm{m}$.

have a power of several tens of $\mathrm{mW}$ at the sample. Images are acquired by scanning the input beams over the sample with two Galvano mirrors (Olympus FluoView 300, IX-71) and focusing with a 60x 1.2 NA (Olympus C.A.R.S.) water immersion objective on the sample. The generated CARS signal is collected in the forward direction by a 0.55 NA condenser lens, filtered by several bandpass filters and detected by a PMT (Hamamatsu R943-02). The SHG signal, generated in the forward direction, is partly backscattered in the sample, collected by the focusing objective, and detected in the non-descanned backward direction. The SHG photons are separated from the input beams by a dichroic mirror and detected by a PMT (Hamamatsu R3896). CARS and SHG images are acquired over an area of 150x150 $\mu \mathrm{m}$, divided into 512x512 pixels. Three dimensional images are obtained by stacking multiple slices along the $z$-axis. Each slice consists of an average of frames, where each frame is acquired in $1.12 \mathrm{~s}$. Images at different vibrational resonances are obtained by temperature tuning of the OPO. 
CARS images of the lipid vibrational resonance $\left(2845 \mathrm{~cm}^{-1}\right)$ are obtained by averaging 5 frames per slice. Due to the high intensity of the lipid droplets compared to the non-resonant background a threshold can be applied to the images. The bone images, at $950 \mathrm{~cm}^{-1}$, are severely influenced by non-resonant background signal. To overcome this problem the spectral behaviour of the resonant and non-resonant signal is exploited. Figure 5.15(d) shows a recording - obtained by rotation of the Lyot filter in the OPO - of the CARS intensity spectrum (890-1030 $\mathrm{cm}^{-1}$ ) of a bone and a bone free (non-resonant background) part in the sample. The spectrum of the non-resonant background can be assumed to be flat over $20 \mathrm{~cm}^{-1}$. The bone spectrum shows a peak at $950 \mathrm{~cm}^{-1}$ and a dip at $970 \mathrm{~cm}^{-1}$. Subtraction of the images obtained at $950 \mathrm{~cm}^{-1}$ and $970 \mathrm{~cm}^{-1}$ results in a background free image showing only the bone features. For example, figure 5.15 (a) shows an image taken at $950 \mathrm{~cm}^{-1}$, figure 5.15(b) shows the same field of view at $970 \mathrm{~cm}^{-1}$, and subtraction results in figure 5.15(c). The first two images are averages of 10 frames. The SHG images revealing the collagen fibrils are obtained by averaging 15 frames per slice for three dimensional and 20 frames for two dimensional images.

\subsubsection{Hypothetical model of how cells grow bone}

Based on the results obtained with Raman spectroscopy, CARS and SHG microscopy a new model for the bone growth is presented. This model is illustrated in figure 5.16.

\section{The proliferation of the cells, the growth of lipid droplets and synthesis of collagen}

Until day 45 the multipotent adult human bone marrow stromal cells show proliferation and differentiation, resulting in an organization of the cells in multiple layers. The cells differentiate towards osteoblasts between days 25 and 30 . The differentiation can be confirmed by the identification of the osteoblast biomarkers with Raman scattering spectroscopy, see figure 5.17 .

Figure 5.18 shows the distribution of lipid droplets in the cells and the collagen distribution between the cell layers, acquired with CARS and SHG microscopy respectively. The measurement from day 25 reveals three layers of cells. The cells closest to the substrate cover slip (bottom) 


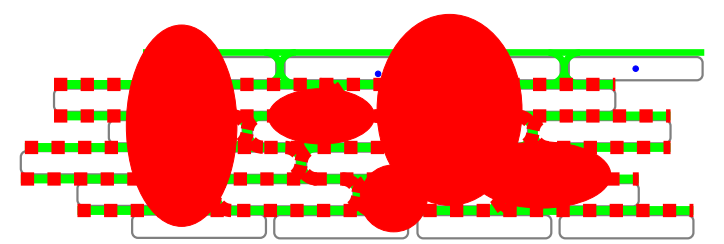

Day 60
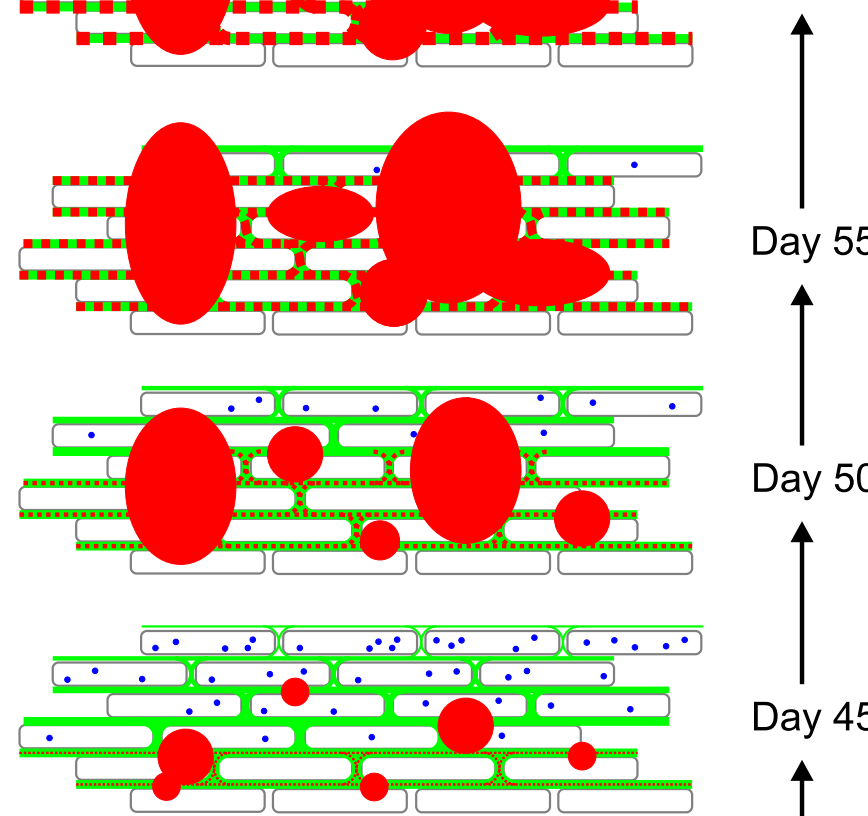

Day 55

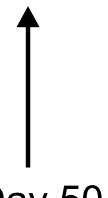

Day 50

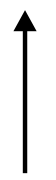

Day 45
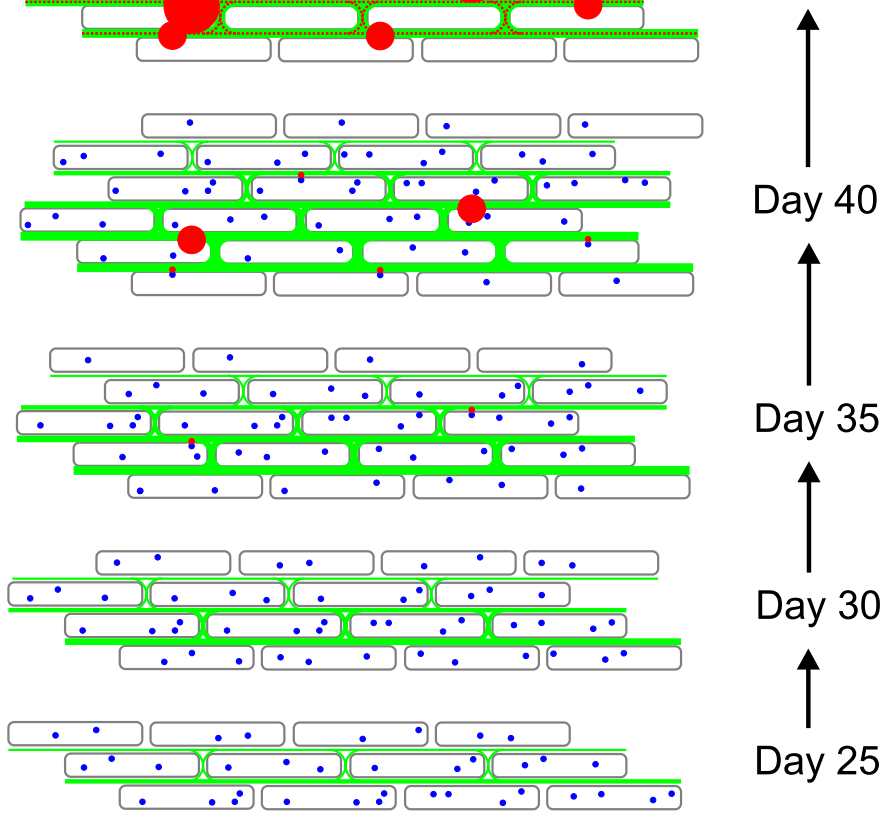

Day 35

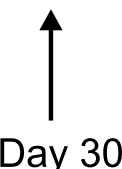

Cells

- Lipid

Day 30

Collagen

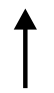

- Bone nodule -... Bone layer

Day 25

Figure 5.16: Illustration of bone development during a 60-day culture. 

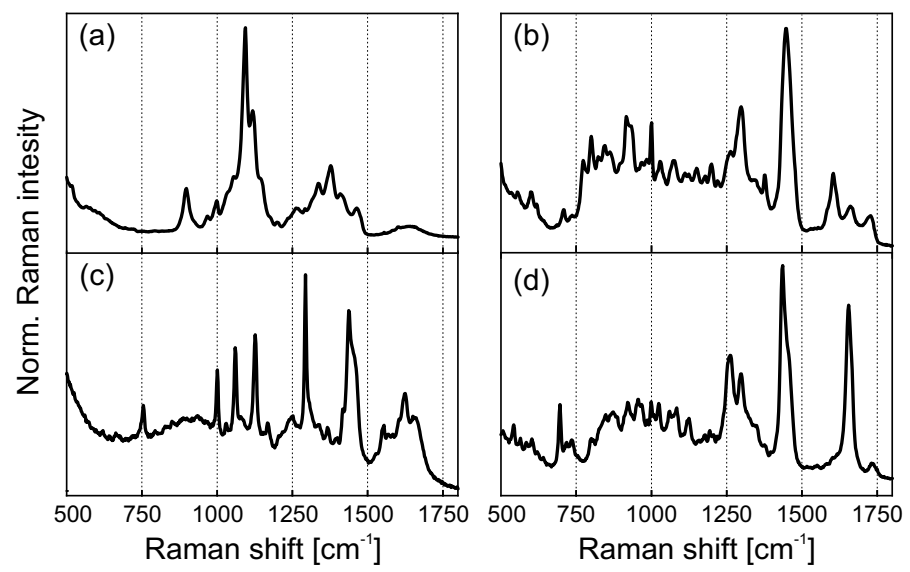

Figure 5.17: Raman spectroscopy showing biomarkers for osteoblast differentiation. (a) glycosaminoglycans, (b) phosphatidylserine, (c) phosphatidic acid, and (d) combination of cholesterol and phosphatidylserine.

show the presence of lipid droplets and a small layer of collagen fibrils on top of these cells. The upper layer of cells shows fewer lipid droplets and almost no collagen. From this observation it can be suggested that the lower cell layers are more differentiated towards preosteoblast cells compared to the cells in the upper layers, which were formed more recently. Older cultures show a growth of layers, where newly formed cells are growing on top of the older cells. On day 45 of culture at least six cell layers could be seen along with a well-developed extracellular matrix of collagen fibrils. The collagen fibrils in the lower layers are much more dense than the newly formed collagen fibrils over the upper layers of cells. The distribution of the lipid droplets in the sample increases from day 25 to day 45 . Days 50 and 55 show just a few lipid droplets in the upper-most layers. Within those days the localization of the lipid droplets shifts up with the newly formed cells. The organization of the collagen fibrils on top of each layer of cells can be seen at different depths in the sample with SHG microscopy, as seen in figure 5.19.

\section{Mineralization around lipid droplets}

The cells are composed of phosphatidic acid, phosphatidylserine and cholesteral as can be seen in the Raman spectra in figure 5.17(b-d). On day 25 and 30 (figure 5.17(d)) Raman spectra reveal the presence of phosphatidylserine and cholesterol in the cells. The lipid droplet may acts as a reservoir to store calcium ions $\left(\mathrm{Ca}^{2+}\right)$. Due to the good crystallographic 


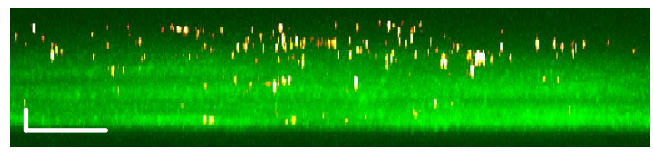

day 45

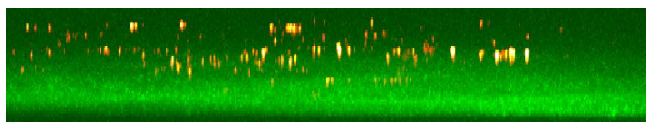

day 40

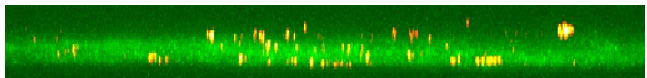

day 35

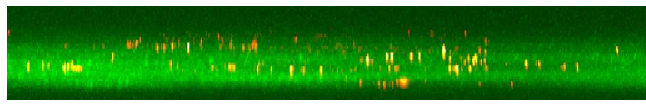

day 30

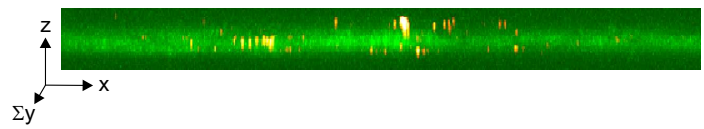

day 25

Figure 5.18: Lipid (CARS, $2845 \mathrm{~cm}^{-1}$, hot colorscale) and collagen (SHG, green colorscale) distribution in the $x, z$ plane, showing the distribution integrated over the y plane of the sample. The non-resonant background is removed by setting an intensity threshold between the intensities of the background and droplets. The bottom of the z-stack shows the sample cover slip. Horizontal scale bar is $20 \mu \mathrm{m}$ and vertical scale bar is $5 \mu \mathrm{m}$.

fit between the crystal structures of hydroxyapatite and cholesterol an epitaxial growth of hydroxyapatite on cholesterol is feasible [112]. On day 35 , see figure 5.21 , a vibrational resonance at $954 \mathrm{~cm}^{-1}$ representing the phosphate band appears at distinct locations in the sample along with lipid spectra. The observation of this phosphate band is a characteristic indication for the initial hydroxyapatite mineral deposition.

On day 40, see figure 5.20, a CARS image is obtained of the bone (red) and the lipids (green). From this image it can be seen that there is some colocalization between lipid droplets and bone. Throughout the sample repeated occurrences suggest the co-localization of bone and lipids. Figure 5.20(b) presents a clearer image of the bone nodule formation. In this figure three different possible stages of bone nodule formation are indicated with circles (I-III). The bone in circle (I) clearly shows the shape and size of several mineralized droplets close to each other. Circle (II) Shows more advanced mineralization, where the drop- 

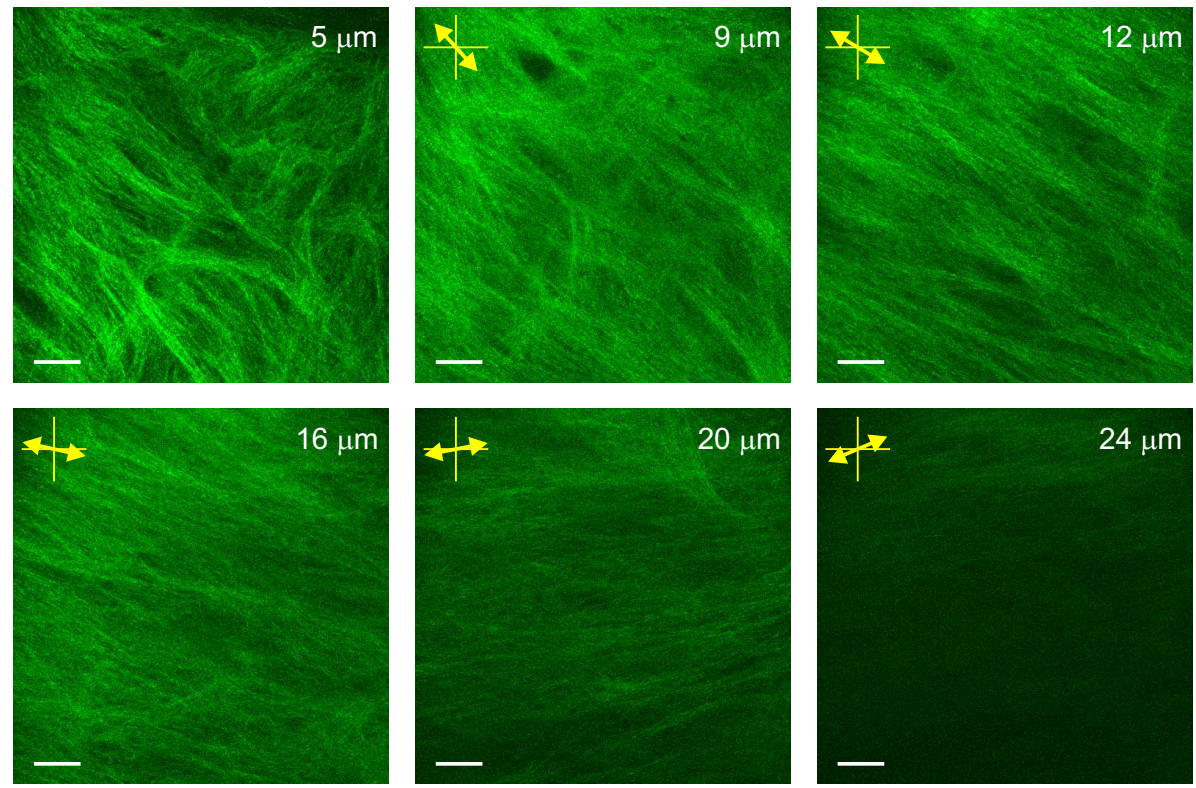

Figure 5.19: SHG microscopy visualizing the collagen fibril arrangement. The arrow indicates the global arrangement direction of the collagen fibrils at a specific depth in the sample. The scale bar is $20 \mu \mathrm{m}$.

lets can still be identified but have significantly grown in size. Circle (III) shows droplets attached to each other; the shapes of the individual droplets are no longer recognizable anymore but fused into the shape of a bone nodule.

The Raman spectrum in figure 5.21 on day 40 of culture shows an increase in intensity of the $954 \mathrm{~cm}^{-1}$ phosphate resonance (hydroxyapatite indicator) and a shift in the resonance from $954 \mathrm{~cm}^{-1}$ to $956 \mathrm{~cm}^{-1}$ compared to day 35. The shift and increase of the vibrational resonance is an indication of the change in the mineral structure and composition toward hydroxyapatite. Further growth shows accretion of hydroxyapatite formation in the cells by the increase of the resonance amplitude. The increase in hydroxyapatite with culture period is accompanied by an appearance of the $1070 \mathrm{~cm}^{-1}$ band from day 45 , indicating a symmetric carbonate vibration [118]. The intensity of this band increases with time, indicating that the phosphates in the hydroxyapatite are substituted by the carbonates from the culture medium. This indicates that there is a conversion of hydroxyapatite to carbonated hydroxyapatite. The formation of carbonated hydroxyapatite results in an increase of the 

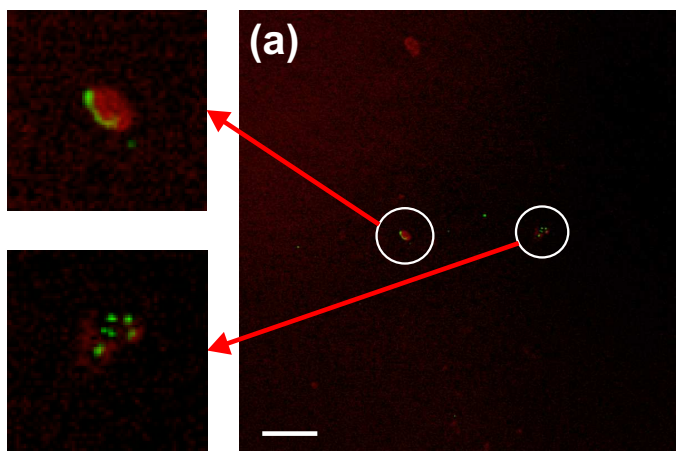

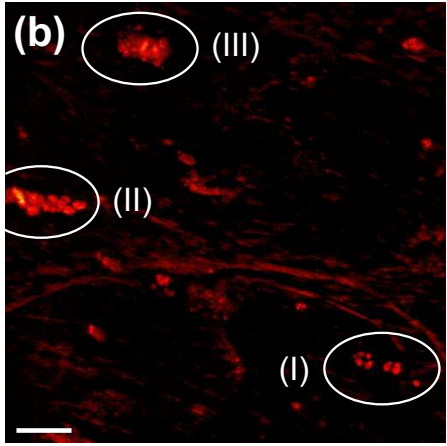

Figure 5.20: (a) Background subtracted image of lipid $\left(2845 \mathrm{~cm}^{-1}\right.$, green) and bone $\left(950 \mathrm{~cm}^{-1}\right.$, red). The lipid is shown with a threshold for suppressing the non-resonant background. The circles indicate colocalization of lipids with bone formation. (b) Background subtracted image of bone $\left(950 \mathrm{~cm}^{-1}\right)$. The image shows the formation of bone nodules from lipid droplets (I-III). The scale bar is $20 \mu \mathrm{m}$.

crystalline nature of the apatite indicated by the shift of the phosphate vibration from $954 \mathrm{~cm}^{-1}$ (day 35) to $959 \mathrm{~cm}^{-1}$ (day 60), and decrease in full width at half maximum from $21.2 \mathrm{~cm}^{-1}$ observed at day 40 to $17.4 \mathrm{~cm}^{-1}$ at day $60[119,120]$. The full width at half maximum of the phosphate vibration seen on day 60 of culture is close to that seen for in vivo bone [121].

\section{Collagen fibrils guiding the bone layer growth}

The collagen fibrils (type-I) are syntheses of the extracellular matrix by the cells, with holes and pores in the interfibrilar spaces. These holes and pores act as small reservoirs for non-collagenous proteins, which are unique to bone and help in mineralization [102]. From day 45, hydroxyapatite is formed on the same location as the collagen fibrils and starts to form a bone layer. In figure 5.22(a-d), CARS microscopy images show the mineralization formation of a bone layer guided by the collagen fibrils on four different locations after 50 days. Figure 5.22(a) shows the start of the bone layer formation, where collagen fibrils are mineralized. This mineralization continues as can be seen in figure 5.22(b). Figure 5.22 (c-d) show an even further state of the mineralization of the collagen fibrils. Figure 5.22(e) shows that the direction of the fibrils overlaps with the direction of bone formation in figure 5.22(c). Figure 5.22(c-d) furthermore show that the bone layer and bone nodules merge into each other at locations that appear more mature. 

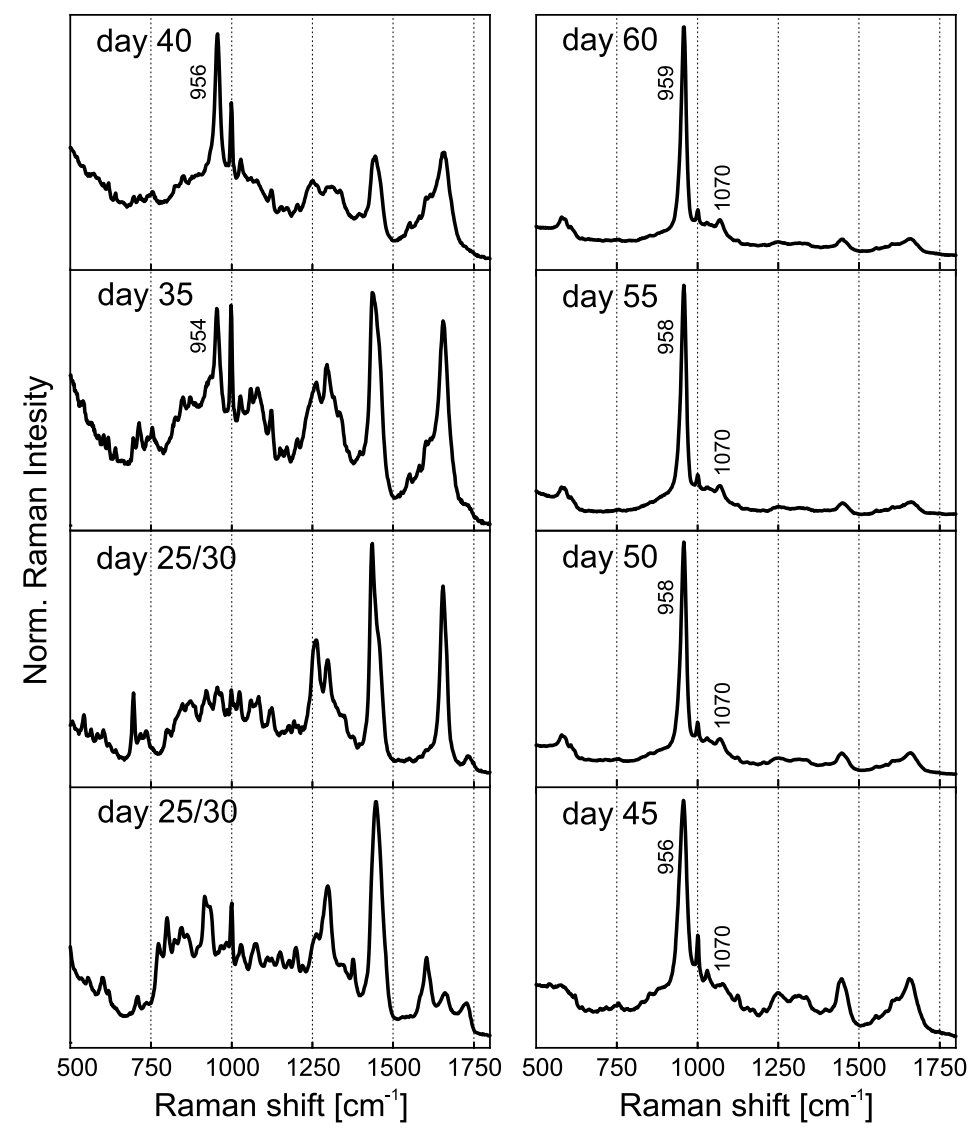

Figure 5.21: Raman specta showing the mineralization and crystallization of bone observed from day 25 until day 60 of culture. On day 25 and 30 of culture a combination of cholesterol and phosphatidylserine is observed. On day 35 of culture, early mineral deposition for phosphates can be observed represented by the band at $954 \mathrm{~cm}^{-1}$. This band gradually shifts towards $959 \mathrm{~cm}^{-1}$ by day 60. The cells show the formation of hydroxyapatite (indicated by phosphate band) and the conversion of hydroxyapatite to carbonated hydroxyapatite represented by the carbonate band at $1070 \mathrm{~cm}^{-1}$.

\section{Three dimensional bone formation}

The early mineralized nodules are independently formed in the extracellular matrix. With time, the collagen fibrils provide mechanical support for the hydroxyapatite deposition, which occurs along the orientation of the collagen fibrils. The combination of the early formed nodules and 

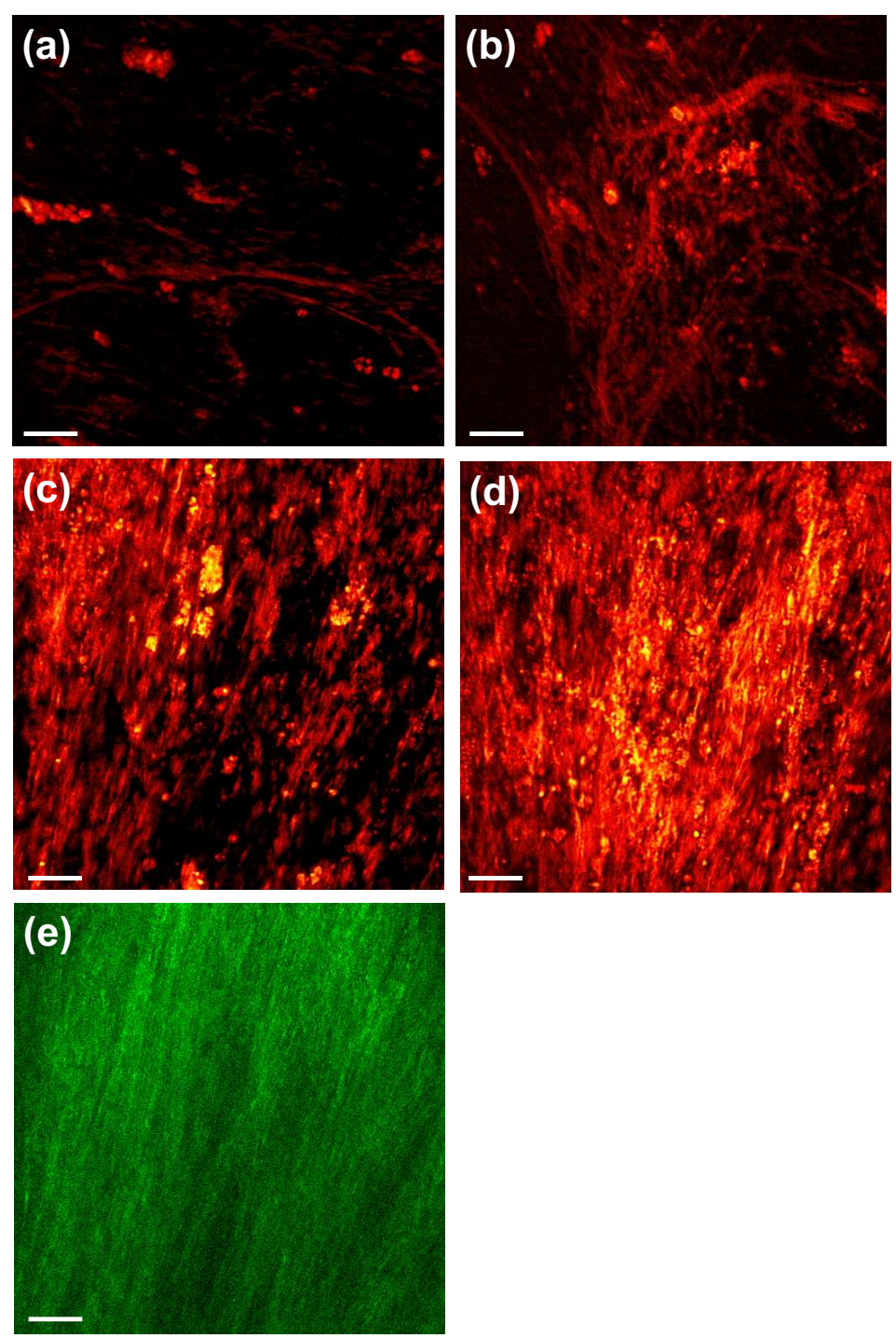

Figure 5.22: Representation of four different stages of bone formation in the 50-day-old culture sample observed at different locations in the sample. (a) Shows the early bone layer formation guided by the collagen fibrils. (b) Further mineralization of the collagen layers. (e) Shows the collagen fibrils of (c) separately. (d) A denser and more mature bone layer. The individually created nodules become part of the bone layer, forming a three dimensional bone matrix. The scale bar is $20 \mu \mathrm{m}$. 

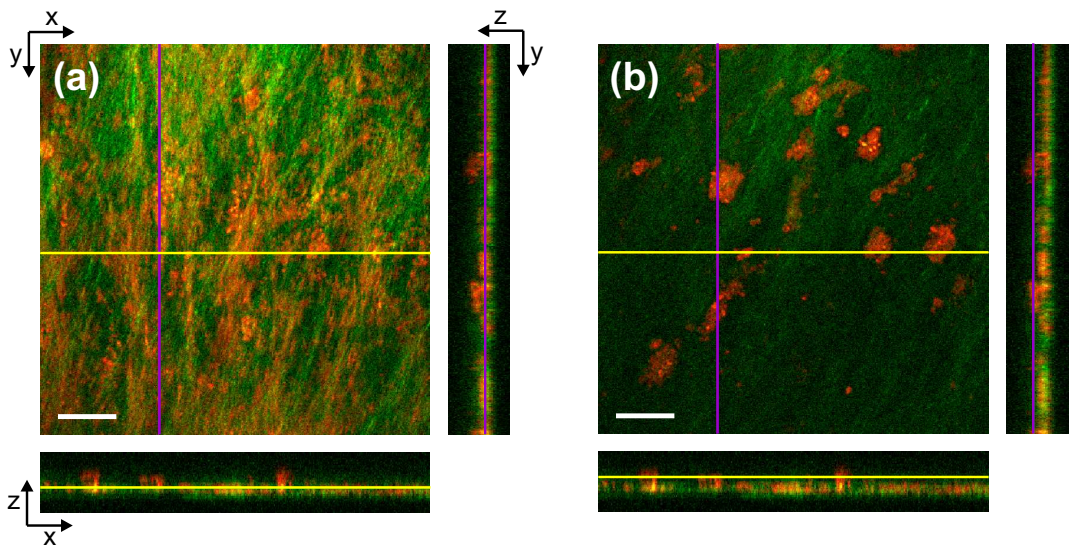

Figure 5.23: (a) $10 \mu \mathrm{m}$ and (b) $13 \mu \mathrm{m}$ depth slice in a 50 day culture sample. Collagen and bone are colored green and red respectively. Lines indicate location and depth of the cross sections. The scale bar is $20 \mu \mathrm{m}$.

the bone layer leads to a three-dimensional structure of bone. Figure 5.23(a) shows a side view of the bone layer with collagen overlap at 10$\mu \mathrm{m}$ depth and figure 5.23(b) (at $13 \mu \mathrm{m}$ depth) shows the bone nodules that do not follow the collagen fibrils and grow in three dimensions. By day 60 of culture, the bone formation embedded with cells and collagen in a three dimensional structure is similar to that of in vivo bone.

\subsubsection{Conclusion}

Multipotent adult human bone marrow stromal cells were cultured towards osteogenic lineage followed by hydroxyapatite mineralization over a 60 day period. Raman spectroscopy, CARS and SHG microscopy were applied to follow these cells and understand how they grow bone. Raman spectroscopy and SHG microscopy are used to confirm the differentiation towards osteogenic lineage. By day 45, at least six layers of cells are formed, embedded within a collagen layer. From day 35 calcium-active phospholipids inside the lipid droplets enable initial mineralization, and later continued growth into three dimensional nodules. From day 40 the concentration of phospholipids in the holes and pores of the collagen fibrils was sufficient to start mineralization. With CARS and SHG microscopy it is shown that the mineralization follows the collagen fibril structure, forming a layer of bone. Furthermore the increase in intensity of the band at $1070 \mathrm{~cm}^{-1}$ indicates an increase of carbonated hydroxyapatite, resulting in an increase in crystallinity of the formed apatite, 
which has been seen with Raman spectroscopy. Day 60 of culture showed three dimensional mineral formations completely embedded with collagen fibrils, with composition similar to in vivo bone. From this study a model of bone growth is constructed. 
Chapter 5 


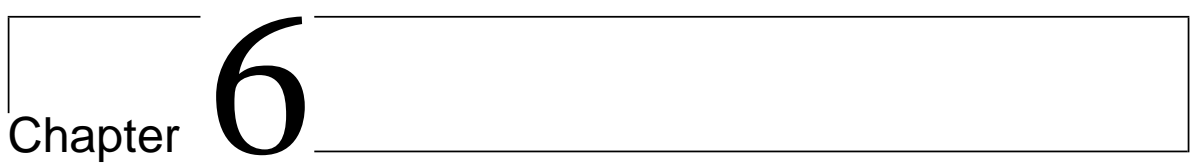

\section{Microscale art}

Throughout this thesis there is a variety of images showing the capabilities of CARS microscopy. Besides the physical and biological information retrieved from the microscopy images, the shapes and assigned colors often form interesting patterns that stimulate the imagination of the reader. In this chapter several of these artistic images are selected, guiding the reader further in the wonderful world of microscale art. 


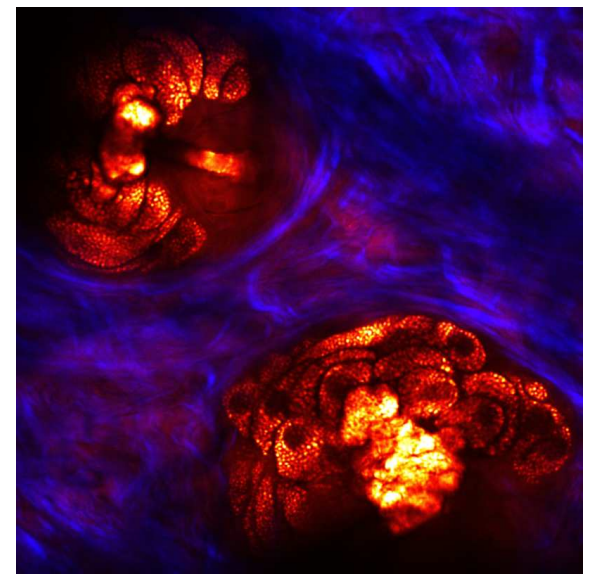

(a) "Stellar gas cloud": A Superposition of a CARS and SHG microscopy image in rat ear. In hot color scale the sebaceous gland can be seen, imaged at a Raman shift of $2845 \mathrm{~cm}^{-1}$. In blue the collagen fibril structure shown. Honorable mention Olympus BioScapes International Digital Imaging Competition 2009

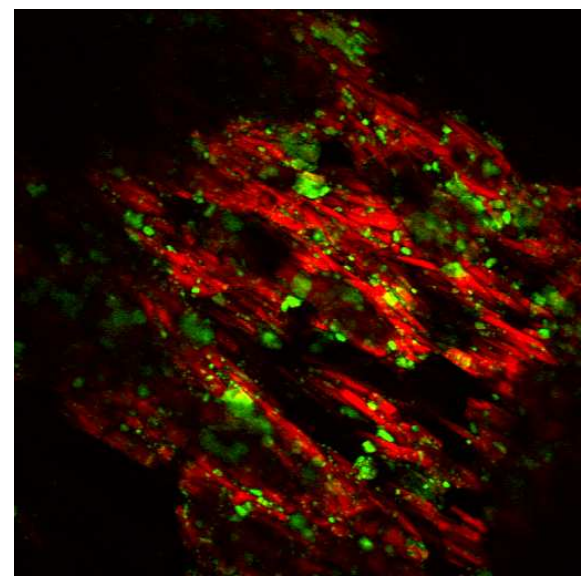

(c) "Alpine landscape": A pharmaceutical tablet surface of the medicine Griseofulvin (antifungal drug) in green (SHG microscopy), packed in a matrix of Mannitol (sugar alcohol) in red (CARS microscopy, $2889 \mathrm{~cm}^{-1}$ ).

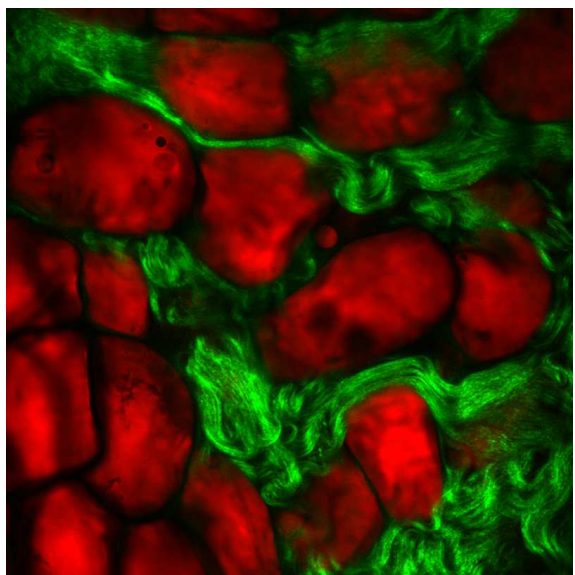

(b) "Pebbles in a river": Beef tissue, where collagen fibrils are represented in green (SHG microscopy) and fat cells in red (CARS microscopy, $2845 \mathrm{~cm}^{-1}$ ). Third prize MESA + photo contest 2009

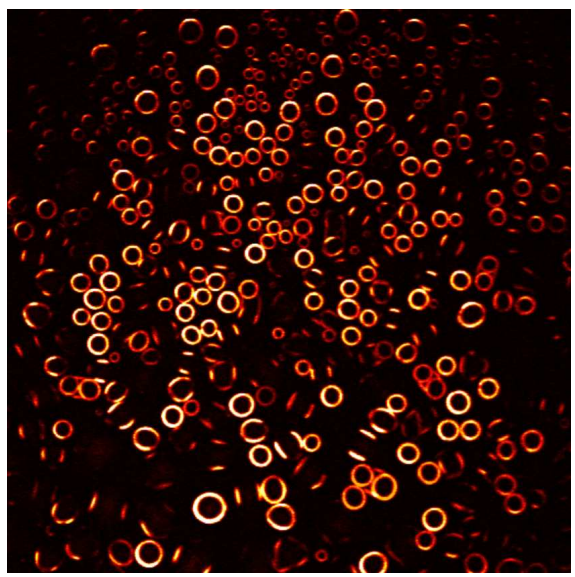

(d) "Magic rings": These ring shapes were observed with CARS microscopy on the edge of a flow cell, where the cover glass is glued to the teflon flow cell with rapid glue. Some rings seem to be connected. 


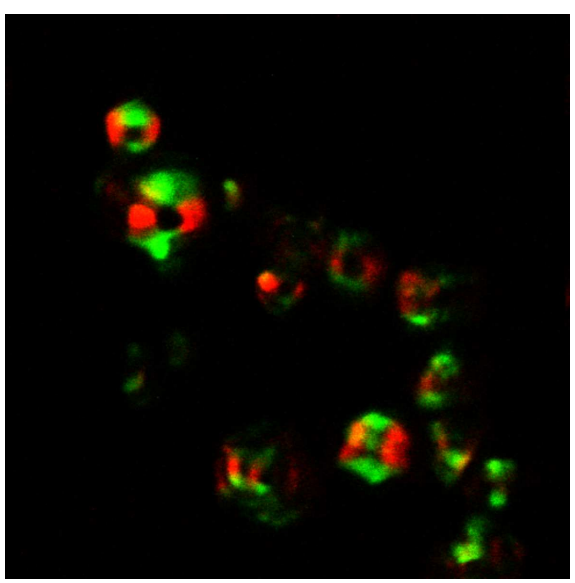

(e) "Spinning discs": Several starch grains imaged with SHG microscopy. The image shows a superposition of the horizontal (red) and vertical polarization (green) of the input beam showing the orientation dependence.

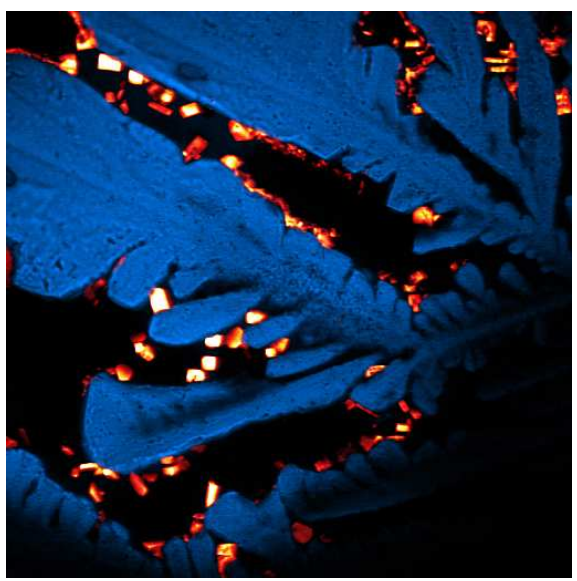

(g) "Oak leaf": Composite of CARS images of a dried protein sample. In the hot color scale, crystals of $\alpha$-synuclein protein light up brightly at $1005 \mathrm{~cm}^{-1}$, attributed to the amino acid phenylalanine. In blue the non-resonant image of crystallized buffer solution is shown.

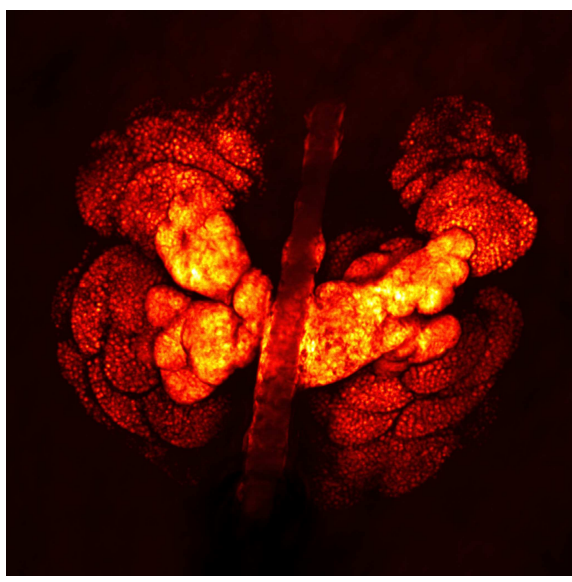

(f) "Junction of fat": CARS microscopy image in a rat ear showing four sebaceous glands attached to a hair, at a Raman shift of $2845 \mathrm{~cm}^{-1}$.

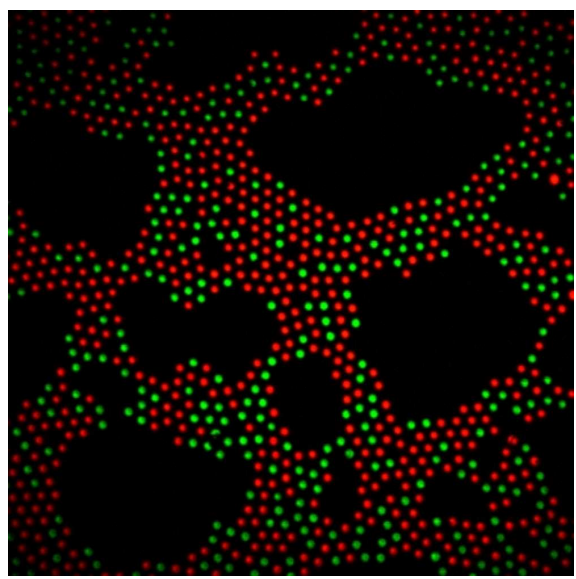

(h) "Ordered marbles": Superposition of CARS images of two different types of plastic $4 \mu \mathrm{m}$ beads, PMMA and PS, obtained at the Raman shifts of 2945 $\mathrm{cm}^{-1}$ and $3050 \mathrm{~cm}^{-1}$ respectively. 


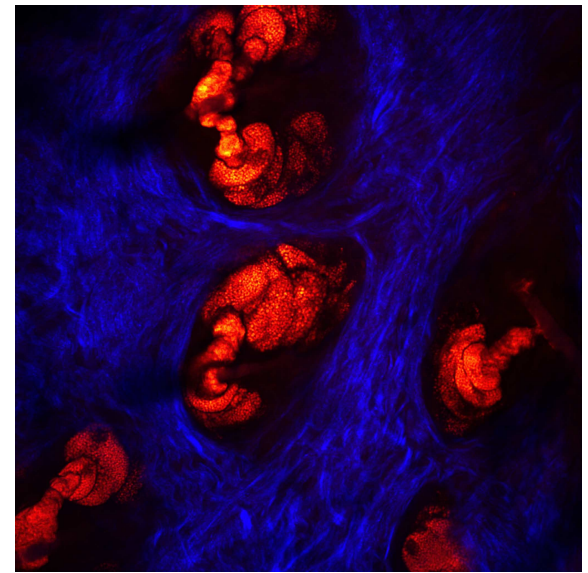

(i) "What is nested": Within a rat ear several sebaceous glands are imaged at the Raman shift of $2845 \mathrm{~cm}^{-1}$ with CARS microscopy (red). Simultaneously the collagen fibril structure is imaged with SHG microscopy (blue).

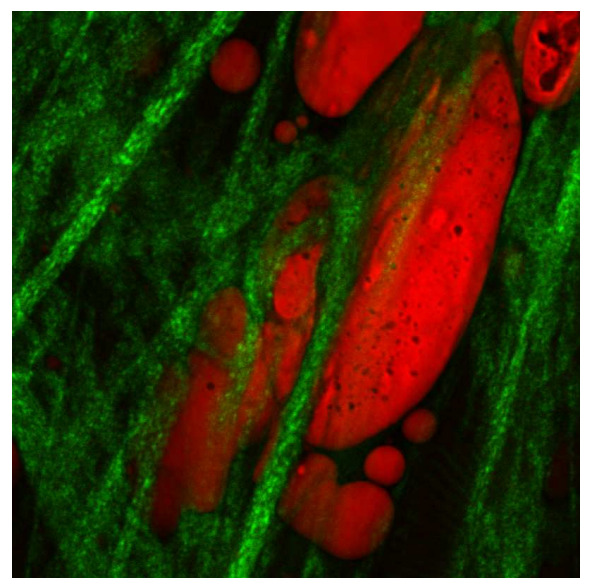

(k) "Hidden in algae": Beef tissue, where collagen fibrils are represented in green (SHG microscopy) and fat cells in red (CARS microscopy, $2845 \mathrm{~cm}^{-1}$ ).

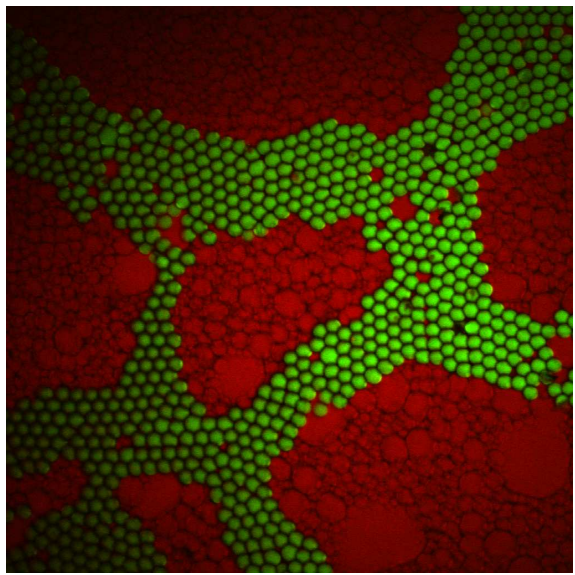

(j) "Honeycomb": A superposition of two CARS images, one of lipid globules in mayonnaise $\left(2845 \mathrm{~cm}^{-1}\right)$ in red and one of polystyrene beads $\left(3055 \mathrm{~cm}^{-1}\right)$ in green.

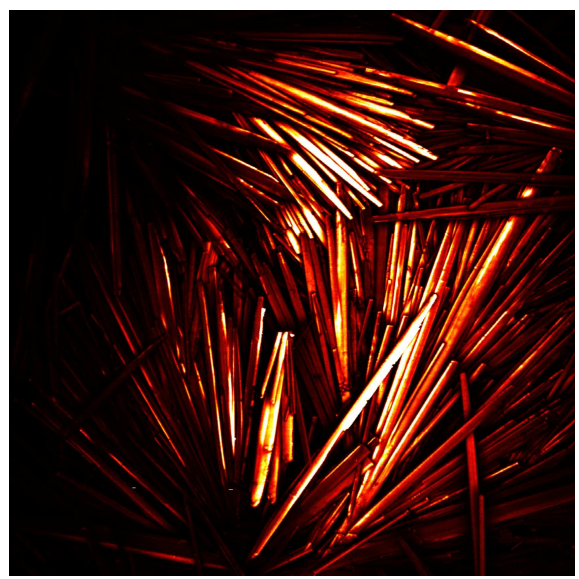

(1) "Wall of crystals": Formation of long needles on a theophylline anhydrate tablet (drug used in therapy for respiratory diseases such as asthma). 

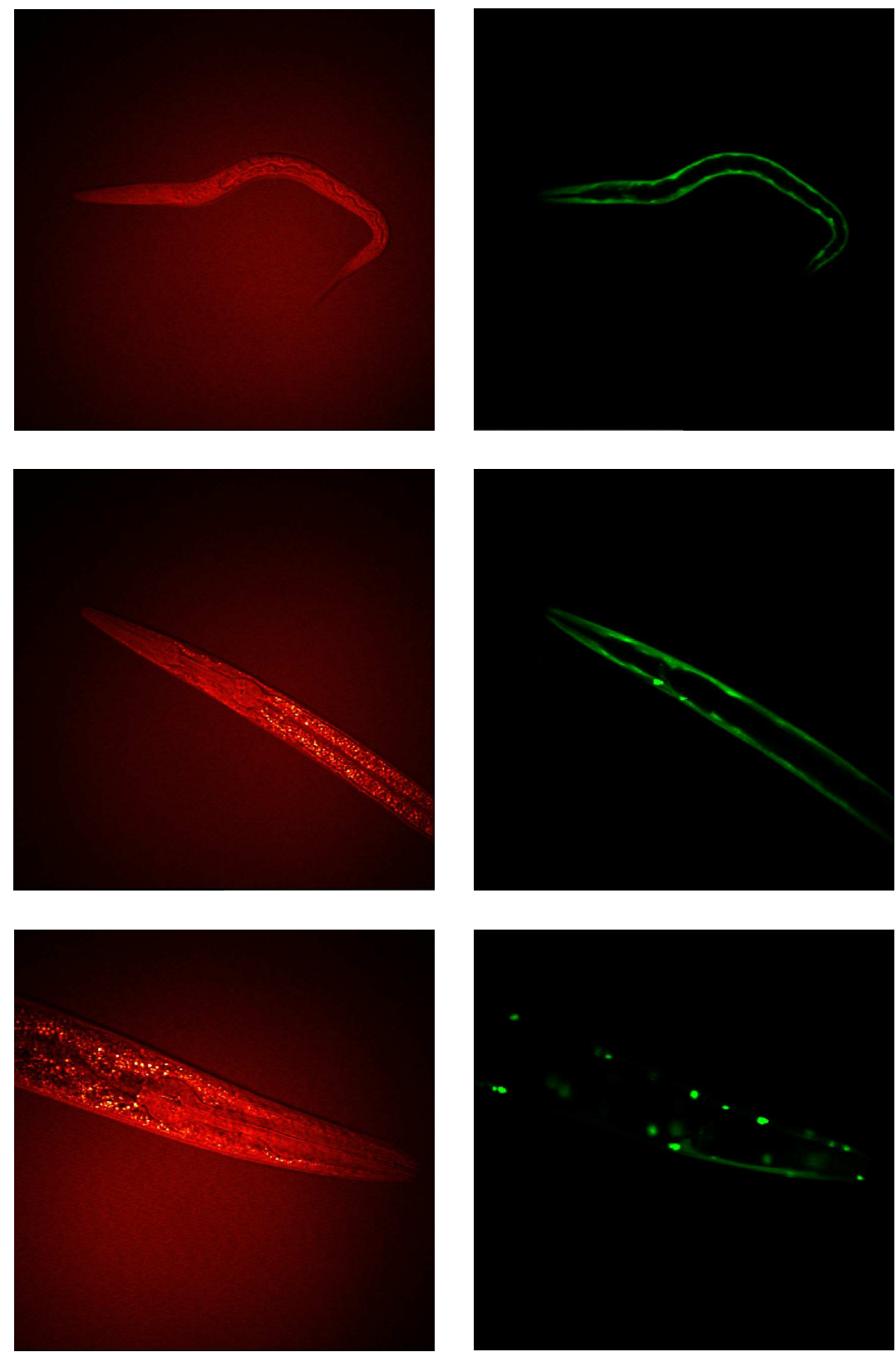

(m) "Protein development during aging in micro-organisms": Three age stages of the C. elegans are shown, from young in the top image to older in the bottom image. The left images represents the CARS image on the lipid vibrational resonance at $2845 \mathrm{~cm}^{-1}$ and the images on the right are the two-photon fluorescence on Q40-YFP. Specific proteins are labeled with Q40-YFP. From the images it can be observed that the proteins aggregate. 

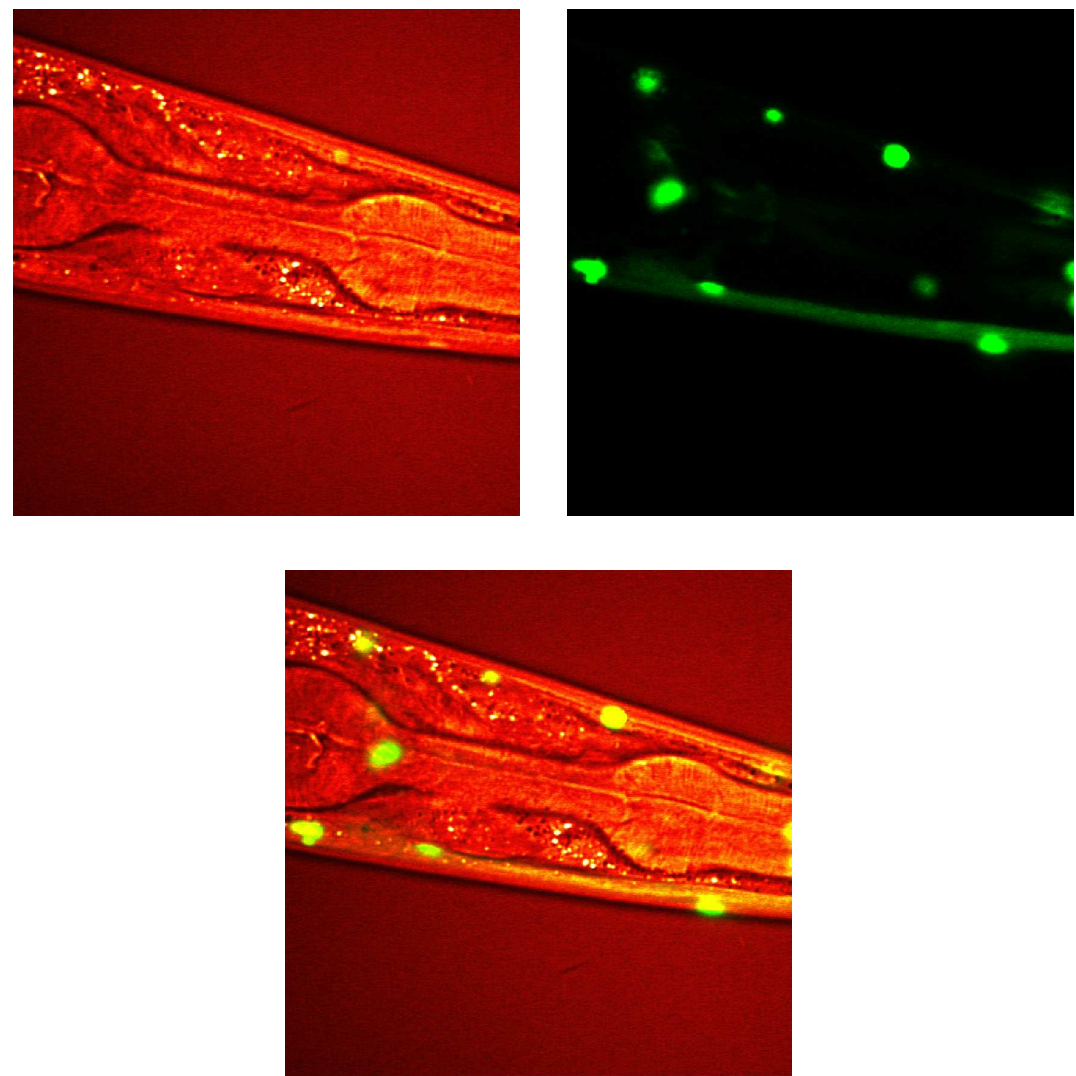

(n) "Possible co-localization": A 7 days old C. elegans. In the first image the lipid vibrational resonance $\left(2845 \mathrm{~cm}^{-1}\right)$ is imaged with CARS microscopy, the second image shows the aggregated polyglutamine YFP (Q40-YFP) protein imaged with two-photon fluorescence and the third image shows a superposition of both images. 


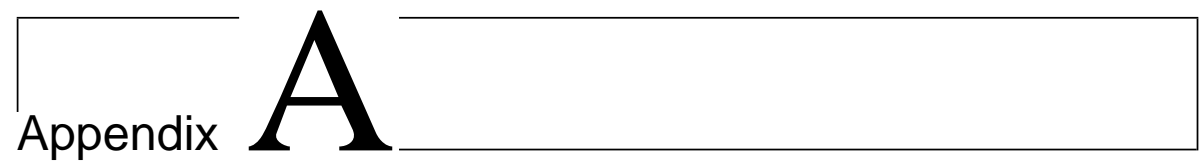

Calculations 


\section{A.1 Noise equivalent Power (NEP)}

An example is given of the calculation of the NEP and what the influence is of the resistor $(\mathrm{R})$ in the transimpedance amplifier.

Photodiode detector:

Detector sensitivity $=0.5 \mathrm{~A} / \mathrm{W}$

Resistor $=1 \mathrm{M} \Omega$

The Johnson noise in the Voltage is given by

$$
V_{\text {noise }}=\sqrt{4 k T R}=127 \mathrm{nV} / \sqrt{\mathrm{Hz}},
$$

where $\mathrm{T}=293 \mathrm{~K}$ and $\mathrm{k}=1.38 \cdot 10^{-23} \mathrm{~J} / \mathrm{K}$.

The noise in the current is given by

$$
I_{\text {noise }}=\frac{V_{\text {noise }}}{R}=127 \mathrm{fA} / \sqrt{\mathrm{Hz}} .
$$

The NEP is now given by

$$
N E P=\frac{I_{\text {noise }[A]}}{\text { detector sensitivity }[A / W]}=254 \mathrm{fW} / \sqrt{H z} .
$$

The bandwidth of the NEP is calculated from the integration time constant of the Lock-in Amplifier (100 ms)

$$
f=\frac{1}{2 \pi \tau}=1.58 \mathrm{~Hz} .
$$

Giving the NEP in $1.58 \mathrm{~Hz}$ bandwidth of

$$
N E P_{1.58 \mathrm{~Hz} \text { bandwidth }}=N E P \cdot \sqrt{f}=319 \mathrm{fW} \text { rms } .
$$

Increasing the detector resistance by a factor 100 to $100 \mathrm{M} \Omega$, the NEP at $1.58 \mathrm{~Hz}$ bandwidth results in $31.9 \mathrm{fW}_{r m s}$. This shows that a 100 times higher resistor results in a $\sqrt{100}=10$ times smaller NEP. 


\section{A.2 Quantum efficiency (QE)}

An example is given of the calculation of the shot noise level and what the influence is of the QE.

The relation between the detector sensitivity and the QE is

$$
\text { detector sensitivity }[A / W]=\frac{Q E \cdot e}{E},
$$

where the electron charge $\mathrm{e}=1.6 \cdot 10^{-19} \mathrm{C}$ and $\mathrm{E}$, the energy of one photon at $800 \mathrm{~nm}$ is given by

$$
E=\frac{h c}{\lambda}=2.49 \cdot 10^{-19} \mathrm{~J},
$$

where $\mathrm{h}=6.6 \cdot 10^{-34} \mathrm{Js}, \mathrm{c}=3 \cdot 10^{8} \mathrm{~m} / \mathrm{s}$ and $\lambda=800 \cdot 10^{-9} \mathrm{~m}$.

Consider two identical detectors with different QE's, of $100 \%$ and $7 \%$ and a local oscillator power of $10 \mathrm{pW}$.

The amount of photons in the local oscillator is $4 \cdot 10^{7}$.

The shot noise on the local oscillator is given by

$$
\text { Shot noise }=\sqrt{\# \text { photons }}=6.3 \cdot 10^{3} .
$$

$4 \cdot 10^{7}$ photons in the local oscillator detected with the $7 \%$ QE detector gives $2.8 \cdot 10^{6}$ electrons with $1.67 \cdot 10^{3}$ electrons shot noise.

The NEP of a detector with a QE of $100 \%=6.3 \cdot 10^{3} / 100 \%=6.3 \cdot 10^{3}$ The NEP of a detector with a QE of $7 \%=1.67 \cdot 10^{3} / 7 \%=2.39 \cdot 10^{4}$

This shows that due to a lower QE a higher NEP is obtained. The NEP in case of shot noise limited detection for a certain $\mathrm{QE}$ can be determined as

$$
N E P_{\text {Shot noise at } Q E}=\frac{N E P_{\text {Shot noise at }} Q E=1}{\sqrt{Q E}} .
$$




\section{Bibliography}

[1] F. Zernike, "Phase contrast, a new method for the microscopic observation of transparent objects," Physica 9, 686-698 (1942).

[2] A. Coons and M. Kaplan, "Localization of antigen in tissue cells: II. improvements in a method for the detection of antigen by means of fluorescent antibody," J. Exp. Med. 91, 1-13 (1950).

[3] M. Chalfie, Y. Tu, G. Euskirchen, W. Ward, and D. Prasher, "Green fluorescent protein as a marker for gene expression," Science 263, 802805 (1994).

[4] R. Yuste, "Fluorescence microscopy," Nat. Methods 2, 902-904 (2005).

[5] C. Raman and K. Krishnan, "A new type of secondary radiation," Nature 121, 501 (1928).

[6] M. Sneep and W. Ubachs, "Direct measurement of the Rayleigh scattering cross section in various gases," J. Quant. Spectrosc. Ra. 92, 293-310 (2005).

[7] E. Smith and G. Dent, Modern Raman spectroscopy: a practical approach (John Wiley \& Sons, 2005).

[8] T. Maiman, "Stimulated optical radiation in ruby," Nature 187, 493494 (1960).

[9] P. Maker and R. Terhune, "Study of optical effects due to an induced polarization third order in the electric field strength," Phys. Rev. 137, A801-A818 (1965).

[10] G. Eesley, ed., Coherent Raman Spectroscopy (Pergamon Press, 1981).

[11] M. Duncan, J. Reintjes, and T. Manuccia, "Scanning coherent anti-Stokes Raman microscope," Opt. Lett. 7, 350-352 (1982). 
[12] A. Voroshilov, "Polarised coherent Raman and saturation micro-Raman studies of biological macromolecules," Ph.D. thesis, University of Twente (1997).

[13] A. Zumbusch, G. Holtom, and X. Xie, "Three-dimensional vibrational imaging by coherent anti-Stokes Raman scattering," Phys. Rev. Lett. 82, 4142-4145 (1999).

[14] N. Bloembergen, Nonlinear Optics (World Scientific Publishing Company, 1996), 4th ed.

[15] R. Hellwarth, "Third-order optical susceptibilities of liquids and solids," Prog. Quant. Electron. 5, 168 (1977).

[16] R. Boyd, Nonlinear optics (Academic Press, 2003), 2nd ed.

[17] J. Cheng and X. Xie, "Coherent anti-Stokes Raman scattering microscopy: Instrumentation, theory, and applications," J. Phys. Chem. B 108, 827-840 (2004).

[18] B. Master and P. So, eds., Handbook of biomedical nonlinear optical microscopy (Oxford University Press, 2008).

[19] Y. Shen, ed., The principles of nonlinear optics (John Wiley and Sons Inc., 1984).

[20] J. Shirley, R. Hall, and A. Eckbreth, "Folded boxCARS for rotational Raman studies," Opt. Lett. 5, 380-382 (1980).

[21] S. Mukamel, Principles of Nonlinear Optical Spectroscopy. (Oxford Univerisity Press, 1995).

[22] H. Kano and H. Hamaguchi, "In-vivo multi-nonlinear optical imaging of a living cell using a supercontinuum light source generated from a photonic crystal fiber," Opt. Express 14, 2798-2804 (2006).

[23] T. Lang, M. Motzkus, H. Frey, and P. Beaud, "High resolution femtosecond coherent anti-Stokes Raman scattering: Determination of rotational constants, molecular anharmonicity, collisional line shifts, and temperature," J. Chem. Phys. 115, 5418-5426 (2001).

[24] C. Evans, E. Potma, M. Puoris'haag, D. Côté, C. Lin, and X. Xie, "Chemical imaging of tissue in vivo with video-rate coherent anti-Stokes Raman scattering microscopy," P. Natl. Acad. Sci. 102, 16807-16812 (2005).

[25] A. Volkmer, J. Cheng, and X. Xie, "Vibrational imaging with high sensitivity via epidetected coherent anti-Stokes Raman scattering microscopy," Phys. Rev. Lett. 87, 023901 (2001).

[26] C. Heinrich, A. Hofer, A. Ritsch, C. Ciardi, S. Bernet, and M. RitschMarte, "Selective imaging of saturated and unsaturated lipids by widefield CARS-microscopy," Opt. Express 16, 2699-2708 (2008). 
[27] O. Burkacky, A. Zumbusch, C. Brackmann, and A. Enejder, "Dual-pump coherent anti-Stokes-Raman scattering microscopy," Opt. Lett. 31, 36563658 (2006).

[28] Y. Yoo, D. Lee, and H. Cho, "Differential two-signal picosecond-pulse coherent anti-Stokes Raman scattering imaging microscopy by using a dualmode optical parametric oscillator," Opt. Lett. 32, 3254-3256 (2007).

[29] F. Ganikhanov, C. Evans, B. Saar, and X. Xie, "High-sensitivity vibrational imaging with frequency modulation coherent anti-Stokes Raman scattering (FM CARS) microscopy," Opt. Lett. 31, 1872-1874 (2006).

[30] J. Kanger, C. Otto, and J. Greve, "Stimulated Raman gain scattering in thin planar dielectric waveguides," Opt. Lett. 20, 2231-2233 (1995).

[31] J. Kanger, C. Otto, and J. Greve, "Stimulated Raman gain spectroscopy of thin layers using dielectric waveguides," J. Phys. Chem. 100, 1629316297 (1996).

[32] P. Nandakumar, A. Kovalev, and A. Volkmer, "Vibrational imaging and microspectroscopies based on coherent anti-Stokes Raman scattering microscopy," Paper presented at the 17th Intl. Conf. on Laser Spectroscopy (ICOLS) (2005).

[33] C. Freudiger, W. Min, B. Saar, S. Lu, G. Holtom, C. He, J. Tsai, J. Kang, and X. Xie, "Label-free biomedical imaging with high sensitivity by stimulated Raman scattering microscopy," Science 322, 1857-1861 (2008).

[34] P. Nandakumar, A. Kovalev, and A. Volkmer, "Vibrational imaging based on stimulated Raman scattering microscopy," New J. Phys. 11, 033026 (2009).

[35] A. Volkmer, L. Book, and X. Xie, "Time-resolved coherent anti-Stokes Raman scattering microscopy: Imaging based on Raman free induction decay," Appl. Phys. Lett. 80, 1505 (2002).

[36] A. Voroshilov, C. Otto, and J. Greve, "Secondary structure of bovine albumin as studied by polarization-sensitive multiplex CARS spectroscopy," Appl. Spectrosc. 50, 78-85 (1996).

[37] J. Cheng, L. Book, and X. Xie, "Polarization coherent anti-Stokes Raman scattering microscopy," Opt. Lett. 26, 1341-1343 (2001).

[38] S. Akhmanov, A. Bunkin, S. Ivanov, and N. Koroteev, "Polarization active Raman spectroscopy and coherent Raman ellipsometry," Sov. Phys. JETP 47, 667 (1978).

[39] G. Marowsky and G. Lüpke, "CARS-background suppression by phasecontrolled nonlinear interferometry," Appl. Phys. B 51, 49-51 (1990).

[40] C. Evans, E. Potma, and X. Xie, "Coherent anti-Stokes Raman scattering spectral interferometry: determination of the real and imaginary components of nonlinear susceptibility chi(3) for vibrational microscopy," Opt. Lett. 29, 2923-2925 (2004). 
[41] E. Potma, C. Evans, and X. Xie, "Heterodyne coherent anti-Stokes Raman scattering (CARS) imaging," Opt. Lett. 31, 241-243 (2006).

[42] H. Kano and H. Hamaguchi, "Ultrabroadband $\left(>2500 \mathrm{~cm}^{-1}\right)$ multiplex coherent anti-Stokes Raman scattering microspectroscopy using a supercontinuum generated from a photonic crystal fiber," Appl. Phys. Lett. 86, 121113 (2005).

[43] H. Rinia, M. Bonn, and M. Müller, "Quantitative multiplex CARS spectroscopy in congested spectral regions," J. Phys. Chem. B 110, 4472-4479 (2006).

[44] E. Vartiainen, "Phase retrieval approach for coherent anti-Stokes Raman scattering spectrum analysis," J. Opt. Soc. Am. B 9, 1209-1214 (1992).

[45] E. Vartiainen, H. Rinia, M. Müller, and M. Bonn, "Direct extraction of Raman line-shapes from congested CARS spectra," Opt. Express 14, 3622-3630 (2006).

[46] W. de Boeij, H. Kanger, G. Lucassen, C. Otto, and J. Greve, "Waveguide CARS spectroscopy: A new method for background suppression, using dielectric layers as a model," Appl. Spectrosc. 47, 723-730 (1993).

[47] V. Krishnamachari and E. Potma, "Focus-engineered coherent antiStokes Raman scattering: A numerical investigation," J. Opt. Soc. Am. A 24, 1138-1147 (2007).

[48] D. Oron, N. Dudovich, D. Yelin, and Y. Silberberg, "Narrow-band coherent anti-Stokes Raman signals from broad-band pulses," Phys. Rev. Lett. 88, 063004 (2002).

[49] T. Hellerer, A. Enejder, and A. Zumbusch, "Spectral focusing: High spectral resolution spectroscopy with broad-bandwidth laser pulses," Appl.Phys. Lett. 85, 25-27 (2004).

[50] A. van Rhijn, S. Postma, J. Korterik, J. Herek, and H. Offerhaus, "Chemically selective imaging by spectral phase shaping for broadband CARS around $3000 \mathrm{~cm}^{-1}$," J. Opt. Soc. Am. B 26, 559-563 (2009).

[51] Y. Fu, H. Wang, R. Shi, and J. Cheng, "Characterization of photodamage in coherent anti-Stokes Raman scattering microscopy," Opt. Express 14, 3942-3951 (2006).

[52] M. Balu, T. Baldacchini, J. Carter, T. Krasieva, R. Zadoyan, and B. Tromberg, "Effect of excitation wavelength on penetration depth in nonlinear optical microscopy of turbid media," J. Biomed. Opt. 14, 010508 (2009).

[53] J. Cheng, A. Volkmer, and X. Xie, "Theoretical and experimental characterization of coherent anti-Stokes Raman scattering microscopy," J. Opt. Soc. Am. B-optical Phys. 19, 1363-1375 (2002). 
[54] G. Lucassen, W. de Boeij, and J. Greve, "Polarization-sensitive CARS of excited-state rhodamine 6g: Induced anisotropy effects on depolarization ratios," Appl. Spectrosc. 47, 1975-1988 (1993).

[55] E. Potma, D. Jones, J. Cheng, X. Xie, and J. Ye, "High-sensitivity coherent anti-Stokes Raman scattering microscopy with two tightly synchronized picosecond lasers," Optics Lett. 27, 1168-1170 (2002).

[56] F. Ganikhanov, S. Carrasco, X. Xie, M. Katz, W. Seitz, and D. Kopf, "Broadly tunable dual-wavelength light source for coherent anti-Stokes Raman scattering microscopy," Opt. Lett. 31, 1292-1294 (2006).

[57] T. Tukker, "A high repetion rate picosecond infrared ligth source for a sum-frequency vibration spectrum," Ph.D. thesis, University of Twente (1998).

[58] T. Tukker, C. Otto, and J. Greve, "Design, optimization, and characterization of a narrow-bandwidth optical parametric oscillator." J. Opt. Soc. Am. B 16, 90-95 (1999).

[59] P. Chimento, M. Jurna, H. Bouwmans, E. Garbacik, L. Hartsuiker, C. Otto, J. Herek, and H. Offerhaus, "High-resolution narrowband CARS spectroscopy in the spectral fingerprint region," J. Raman Spectrosc. 40, 1229-1233 (2009).

[60] D. Preuss and J. Gole, "Three-stage birefringent filter tuning smoothly over the visible region: theoretical treatment and experimental design," Appl. Opt. 19, 702-710 (1980).

[61] F. Lu, W. Zheng, and Z. Huang, "Heterodyne polarization coherent anti-Stokes Raman scattering microscopy," Appl. Phys. Lett. 92, 123901 (2008).

[62] M. Jurna, J. Korterik, H. Offerhaus, and C. Otto, "Noncritical phasematched lithium triborate optical parametric oscillator for high resolution coherent anti-Stokes Raman scattering spectroscopy and microscopy," Appl. Phys. Lett. 89, 251116 (2006).

[63] W. Koechner, ed., Solid-state laser engineering, vol. 1 (Springer, 1996), 4 th ed.

[64] Y. Chen, D. de Bruin, C. Kerbage, and J. de Boer, "Spectrally balanced detection for optical frequency domain imaging," Opt. Express 15, 16390-16399 (2007).

[65] B. Bouma and G. Tearney, Handbook of Optical Coherence Tomography (Informa Healthcare, 2001).

[66] M. Jurna, J. Korterik, C. Otto, J. Herek, and H. Offerhaus, "Vibrational phase contrast microscopy by use of coherent anti-Stokes Raman scattering," Phys. Rev. Lett. 103, 043905 (2009). 
[67] P. Chen, "Rejection of background light using single-wavelength detection in nonlinear Raman spectroscopy," Appl. Spectrosc. 51, 376-379 (1997).

[68] X. Nan, J. Cheng, and X. Xie, "Vibrational imaging of lipid droplets in live fibroblast cells with coherent anti-Stokes Raman scattering microscopy," J. Lipid Res. 44, 2202-2208 (2003).

[69] X. Nan, E. Potma, and X. Xie, "Nonperturbative chemical imaging of organelle transport in living cells with coherent anti-Stokes Raman scattering microscopy," Biophys. J. 91, 728-735 (2006).

[70] T. Hellerer, C. Axang, C. Brackmann, P. Hillertz, M. Pilon, and A. Enejder, "Monitoring of lipid storage in caenorhabditis elegans using coherent anti-Stokes Raman scattering (CARS) microscopy," P. Natl. Acad. Sci. 104, 14658-14663 (2007).

[71] Y. Fu, T. Huff, H. Wang, J. Cheng, and H. Wang, "Ex vivo and in vivo imaging of myelin fibers in mouse brain by coherent anti-Stokes Raman scattering microscopy," Opt. Express 16, 19396-19409 (2008).

[72] H. Wang, I. Langohr, M. Sturek, and J. Cheng, "Imaging and quantitative analysis of atherosclerotic lesions by CARS-based multimodal nonlinear optical microscopy," Arterioscl. Throm. Vas. 29, 1342-1348 (2009).

[73] C. Evans, X. Xu, S. Kesari, X. Xie, S. Wong, and G. Young, "Chemicallyselective imaging of brain structures with CARS microscopy," Opt. Express 15, 12076-12087 (2007).

[74] M. Zimmerley, C. Lin, D. Oertel, J. Marsh, J. Ward, and E. Potma, "Quantitative detection of chemical compounds in human hair with coherent anti-Stokes Raman scattering," J. Biomed. Opt. 14, 044019 (2009).

[75] A. Wright, S. Poland, J. Girkin, C. Freudiger, C. Evans, and X. Xie, "Adaptive optics for enhanced signal in CARS microscopy," Opt. Express 15, 18209-18219 (2007).

[76] H. Chen, H. Wang, M. Slipchenko, Y. Jung, Y. Sh, J. Zhu, K. Buhman, and J. Cheng, "A multimodal platform for nonlinear optical microscopy and microspectroscopy," Opt. Express 17, 1282-1290 (2009).

[77] P. Franken, A. Hill, C. Peters, and G. Weinreich, "Generation of optical harmonics," Phys. Rev. Lett. 7, 118119 (1961).

[78] I. Freund and M. Deutsch, "Second-harmonic microscopy of biological tissue," Opt. Lett. 11, 94-96 (1989).

[79] G. New and J. Ward, "Optical third-harmonic generation in gases," Phys. Rev. Lett. 19, 556-559 (1967). 
[80] A. Millard, P. Wiseman, D. Fittinghoff, K. Wilson, J. Squier, and M. Müller, "Third-harmonic generation microscopy by use of a compact, femtosecond fiber laser source." Appl. Opt. 38, 7393-7 (1999).

[81] R. Schaller, J. Johnson, and R. Saykally, "Nonlinear chemical imaging microscopy: near-field third harmonic generation imaging of human red blood cells." Anal. Chem. 72, 53615364 (2000).

[82] D. Débarre, W. Supatto, P. A.M., A. Fabre, T. Tordjmann, L. Combettes, M. Schanne-Klein, and E. Beaurepaire, "Imaging lipid bodies in cells and tissues using third-harmonic generation microscopy." Nat. Methods 3, 47-53 (2006).

[83] S. Tang, T. Krasieva, Z. Chen, G. Tempea, and B. Tromberg, "Effect of pulse duration on two-photon excited fluorescence and second harmonic generation in nonlinear optical microscopy," J. Biomed. Opt. 11, 020501 (2006).

[84] H. Press, "Therapeutic tablets (U.S. patent. 2.953.497)," (1960).

[85] H. Brittain, Polymorphism in pharmaceutical solids (Marcel Dekker Inc., New York, 1999).

[86] E. Kang, H. Wang, I. Keun Kwon, J. Robinson, K. Park, and J. Cheng, "In situ visualization of paclitaxel distribution and release by coherent anti-Stokes Raman scattering microscopy," Anal. Chem. 78, 8036-8043 (2006).

[87] H. Wang, N. Bao, T. Le, C. Lu, and J. Cheng, "Microfluidic CARS cytometry," Opt. Express 16, 5782-5789 (2008).

[88] E. Kang, J. Robinson, K. Park, and J. Cheng, "Paclitaxel distribution in poly(ethylene glycol) / poly(lactide-co-glycolic acid) blends and its release visualized by coherent anti-Stokes Raman scattering microscopy," J. Control. Release 122, 261-268 (2007).

[89] M. Windbergs, C. Strachan, and P. Kleinebudde, "Understanding the solid-state behaviour of triglyceride solid lipid extrudates and its influence on dissolution," Eur. J. Pharm. Biopharm. 71, 80-87 (2009).

[90] S. Bresson, M. El Marssi, and B. Khelifa, "Raman spectroscopy investigation of various saturated monoacid triglycerides," Chem. Phys. Lipids 134, 119129 (2005).

[91] M. Nolasco, A. Amado, and P. Ribeiro-Claro, "Computationally-assisted approach to the vibrational spectra of molecular crystals: study of hydrogen-bonding and pseudo-polymorphism," Chem. Phys. Chem. 7, 2150-2161 (2006).

[92] A. Amado, M. Nolasco, and P. Ribeiro-Claro, "Probing pseudopolymorphic transitions in pharmaceutical solids using Raman spectroscopy: Hydration and dehydration of theophylline," J. Pharm. Sci. 96, 1366-1379 (2007). 
[93] L. Peltonen, P. Liljerothb, T. Heikkiläa, K. Kontturib, and J. Hirvonena, "Dissolution testing of acetylsalicylic acid by a channel flow method correlation to usp basket and intrinsic dissolution methods," J. Eur. J. Pharm. Sci. 19, 395401 (2003).

[94] C. Reitz, C. Strachan, and P. Kleinebudde, "Solid lipid extrudates as sustained release matrices: The effect of surface structure on drug release properties," Eur. J. Pharm. Sci. 35, 335-343 (2008).

[95] J. Aaltonen, P. Heinänen, L. Peltonen, H. Kortejärvi, V. Tanninen, L. Christiansen, J. Hirvonen, J. Yliruusi, and J. Rantanen, "In-situ measurement of solvent-mediated phase transformations during dissolution testing," J. Pharm. Sci. 95, 2730-2737 (2006).

[96] S. Debnath, P. Predecki, and R. Suryanarayanan, "Use of glancing angle $\mathrm{x}$-ray powder diffractometry to depth-profile phase transformations during dissolution of indomethacin and theophylline tablets," Pharm. Res. 21, 149-159 (2004).

[97] H. Ando, M. Ishii, M. Kayano, and H. Ozawa, "Effect of moisture on crystallization of theophylline in tablets," Drug Dev. Ind. Pharm. 18, 453-467 (1992).

[98] J. de Smidt, J. Fokkens, H. Grijseels, and J. Crommelin, "Dissolution of theophylline monohydrate and anhydrous theophylline in buffer solutions," J. Pharm. Sci. 75, 497-501 (1986).

[99] N. Peppas, P. Bures, W. Leobandung, and H. Ichikawa, "Hydrogels in pharmaceutical formulations," Eur. J. Pharm. Biopharm. 50, 27-46 (2000).

[100] M. Pittenger, A. Mackay, S. Beck, R. Jaiswal, R. Douglas, J. Mosca, M. Moorman, D. Simonetti, S. Craig, and D. Marshak, "Multilineage potential of adult human mesenchymal stem cells," Science 284, 143147 (1999).

[101] S. Kale, S. Biermann, C. Edwards, C. Tarnowski, M. Morris, and M. Long, "Three dimensional cellular development is essential for ex vivo formation of human bone," Nat. Biotechnol. 18, 954-8 (2000).

[102] M. Favus, ed., Primer on the Metabolic Bone Diseases And Disorders of Mineral Metabolism, (Lippincott Williams \& Wilkins, 2006), 6th ed.

[103] W. Michael, "Osteogenesis and bone-marrow-derived cells," Blood Cell Mol. Dis. 27, 677-690 (2001).

[104] A. I. Caplan, "Adult mesenchymal stem cells for tissue engineering versus regenerative medicine," J. Cell. Physiol. 213, 341-347 (2007).

[105] H. Anderson, "Molecular biology of matrix vesicles," Clin. Orthop. Relat. Res. 314, 266-80 (1995). 
[106] R. Felix and H. Fleisch, "The role of matrix vesicles in calcification," Calcif. Tissue Res. 21, 344-8 (1976).

[107] B. Boyan, Z. Schwartz, L. Swain, and A. Khare, "Role of lipids in calcification of cartilage," Anat. Rec. 224, 211-19 (1989).

[108] B. Zimmermann, H. Wachtel, and C. Noppe, "Patterns of mineralization in vitro," Cell Tissue Res. 263, 483-93 (1991).

[109] F. Parhami, N. Mody, N. Gharavi, Y. Ballard, A.J. Tintut, and L. Demer, "Role of the cholesterol biosynthetic pathway in osteoblastic differentiation of marrow stromal cells," J. Bone Miner. Res. 17, 1997-2003 (2002).

[110] G. Viccica, E. Vignali, and C. Marcocci, "Role of the cholesterol biosynthetic pathway in osteoblastic differentiation," J. Endocrinol Invest 30, 8-12 (2007).

[111] B. Craven, "Crystal structure of cholesterol monohydrate," Nature 260, 727-9 (1976).

[112] D. Laird, M. Mucalo, and Y. Yokogawa, "Growth of calcium hydroxyapatite (Ca-HAp) on cholesterol and cholestanol crystals from a simulated body fluid: a possible insight into the pathological calcifications associated with atherosclerosis," J. Colloid Interface Sci. 295, 348-363 (2006).

[113] S. Stewart, D. Shea, C. Tarnowski, M. Morris, D. Wang, R. Franceschi, D. Lin, and E. Keller, "Trends in early mineralization of murine calvarial osteoblastic cultures: A Raman microscopic study," J. Raman Spectrosc. 33, 536-543 (2002).

[114] L. Gerstenfeld, S. Chipman, C. Kelly, K. Hodgens, D. Lee, and W. Landis, "Collagen expression, ultrastructural assembly, and mineralization in cultures of chicken embryo osteoblasts," J. Cell Biol. 106, 979-89 (1988).

[115] L. Kuhn, Y. Wu, C. Rey, L. Gerstenfeld, M. Grynpas, J. Ackerman, H. Kim, and M. Glimcher, "Structure, composition, and maturation of newly deposited calciumphosphate crystals in chicken osteoblast cell cultures," J. Bone Miner. Res. 15, 1301-1309 (2000).

[116] S. Xu and J. Yu, "Beneath the minerals, a layer of round lipid particles was identified to mediate collagen calcification in compact bone formation," Biophys. J. 91, 4221-4229 (2006).

[117] V. Pully and C. Otto, "The intensity of the $1602 \mathrm{~cm}^{-1}$ band in human cells is related to mitochondrial activity," J. Raman Spectrosc. 40, 473475 (2009).

[118] H. K. Chiang, F. Peng, S. Hung, and Y. Feng, "In situ Raman spectroscopic monitoring of hydroxyapatite as human mesenchymal stem cells differentiate into osteoblasts," J. Raman Spectrosc. 40, 546 - 549 (2008).

[119] J. Timlin, A. Carden, and M. Morris, "Chemical microstructure of cortical bone probed by Raman transects," Appl. Spectrosc. 53, 1429-1435 (1999). 
[120] J. Timlin, A. Carden, M. Morris, R. Rajachar, and D. Kohn, "Raman spectroscopic imaging markers for fatigue-related microdamage in bovine bone," Anal. Chem. 72, 2229-2236 (2000).

[121] E. Gentleman, R. Swain, N. Evans, S. Boonrungsiman, M. Jell, G. Ball, T. Shean, M. Oyen, A. Porter, and M. Stevens, "Comparative materials differences revealed in engineered bone as a function of cell-specific differentiation," Nat. Mater. 8, 763-770 (2009). 


\section{Summary}

This thesis describes a new technique that improves specificity, selectivity and sensitivity in coherent anti-Stokes Raman scattering (CARS) microscopy. CARS microscopy is a nonlinear optical technique that utilizes the individual and group specific bonds of molecules, sometimes referred to as the 'fingerprint' of a molecule, to obtain chemically specific imaging of a sample. A single 'unique' vibrational resonance within the molecule can be addressed and the locations of this resonance within the sample can be imaged. This nonlinear vibrational microscopy technique provides a noninvasive method to identify structures of varying chemical composition.

Despite the label-free detection, high collection efficiencies and absence of interference from one-photon autofluorescence, CARS suffers from a frequency-independent background signal, the 'non-resonant background'. In mixtures, the resonant CARS signal of less abundant constituents may be overwhelmed by the non-resonant background, preventing detection of resonant molecules.

Chapter two describes the theory behind the CARS process, the nonlinear susceptibility, and its relationship to the resonant and non-resonant contributions to the CARS signal. Established methods of reducing the non-resonant background are discussed briefly.

Chapter three presents heterodyne detection at the shot noise level. A cascaded phase preserving chain is used for the generation of a wellcontrolled and stable local oscillator field. The heterodyne detection yields an amplitude and a phase. The amplitude is interferometically 
amplified so that the detection sensitivity is improved by more than 3 orders of magnitude for detection with a photodiode. The phase detection allows for a rejection of the non-resonant background signal, without reduction of the resonant signal, so that the selectivity is also improved.

Chapter four demonstrates that the vibrational phase of the oscillators in the focal volume can be obtained by the subtraction of the local excitation phase from the heterodyne phase. We call this technique "Vibrational Phase Contrast CARS". The vibrational phase is measured on a point-by-point basis and takes into account the refractive index changes in the sample, the phase curvature over the field-of-view and interferometric instabilities during the measurement. This detection of the phase of the vibrational motion can be regarded as a vibrational extension of the linear (refractive index) phase contrast microscopy introduced by Zernike in 1933. A third order cascaded phase-preserving chain is proposed that would further simplify the detection. The possibilities for multi-compound analysis from measurements at a single wavelength are explored. Measurements of dissolution, mixing and multi-component samples are presented.

In chapter five CARS microscopy is applied to biology, pharmacology and food, where chemical selectivity and label-free detection are essential. Other nonlinear signals, generated simultaneously, can be used to obtain more information from the sample; CARS, second harmonic generation microscopy and spontaneous Raman spectroscopy are used side-by-side. Two in-depth studies are presented on the dissolution behavior of a drug and on early bone growth in cells.

Chapter six contains a variety of images showing the capabilities of CARS microscopy. Besides the physical and biological information retrieved from the microscopy images, the shapes and assigned colors often form interesting patterns that stimulate the imagination of the reader. Several of these artistic images are selected, guiding the reader through the wonderful world of microscale art. 


\section{Samenvatting}

Dit proefschrift beschrijft de verbetering van de specificiteit, selectiviteit en gevoeligheid van coherente anti-Stokes Raman verstrooing (CARS) microscopie. CARS microscopie is een niet-lineaire optische techniek die gebruik maakt van de vibraties van een molecuul. Deze vibraties zijn kenmerkend voor een molecuul en worden daarom vaak aangeduid als de 'vingerafdruk' van het molecuul. Door een enkele 'unieke' vibratie te gebruiken kan een chemisch specifieke afbeelding worden gemaakt van het molecuul,. Daarbij hoeven geen extra stoffen te worden toegevoegd, oftewel de techniek is 'label-free'. Het is bovendien niet-invasief, waardoor het een uitstekende techniek is om moleculen te bekijken in hun "natuurlijke" staat.

CARS wordt niet gehinderd door inmenging van één-foton autofluorescentie en het signaal kan daarnaast efficint worden gedetecteerd. Het CARS signaal bevat echter ook een frequentie-onafhankelijk achtergrondsignaal. Dit achtergrondsignaal wordt de 'niet-resonante achtergrond' genoemd. In mengsels van verschillende moleculen kan het resonante (gewenste) CARS signaal van één soort moleculen worden overweldigd door het niet-resonante achtergrondsignaal van andere aanwezige moleculen. Dit kan ertoe leiden dat lage concetraties van resonante moleculen niet gedetecteerd kunnen worden. Om die concetraties toch te kunnen detecteren moet de selectiviteit worden verbeterd.

Hoofdstuk één geeft een inleiding over CARS microscopie en de toepassingen daarvan.

Hoofdstuk twee beschrijft de theorie achter het CARS mechanisme, de niet-lineaire susceptabiliteit, en hoe deze gerelateerd is aan de resonante 
en de niet-resonante bijdrage aan het CARS signaal. Verschillende methoden voor het verminderen van de niet-resonante achtergrond worden kort besproken.

Hoofdstuk drie introduceert heterodyne detectie. Een fase-behoudende keten wordt gebruikt voor het creëren van een goed gecontroleerde en stabiele lokale oscillator voor heterodyne detectie. Daarmee wordt zowel amplitude- als fase-informatie van het CARS signaal verkregen. De amplitude wordt interferometrisch versterkt wat leidt tot een verbetering in detectiegevoeligheid van meer dan 3 orde groottes voor detectie met een fotodiode. De gevoeligheid wordt zodaning verbeterd dat deze het hagelruis-niveau van het signaal bereikt. De fasedetectie maakt het mogelijk om de niet-resonante achtergrond te verwijderen zonder verlies van het resonante signaal wat leidt tot een sterk verbeterde selectiviteit.

Hoofdstuk vier beschrijft hoe de pure (vibrationele) fase van de moleculen kan worden verkregen door de lokale excitatie-fase af te trekken van de fase die wordt verkregen met hetrodyne detectie. Voor deze techniek introduceren we de naam "Vibrationele Fase Contrast CARS". De vibrationele fase wordt punt-voor-punt gemeten en compenseert verschillen in brekingsindex, fase kromming in de microscoop en interferometrische instabiliteiten tijdens de meting. De methode kan worden beschouwd als een vibrationele uitbreiding op de lineaire (brekingsindex) fasecontrast microscopie die is geïntroduceerd door Zernike in 1933. Het gebruik van de fase voor de analyse van mengsels wordt verder uitgewerkt. Hierdoor wordt de selectiviteit verder verbeterd voor metingen bij een enkele golflengte. Tevens wordt een werkwijze gebaseerd op een derde-orde fasebehoudende keten voorgesteld, waarmee de detectie nog verder kan worden versimpeld.

In hoofdstuk vijf wordt beschreven hoe CARS microscopie is toegepast in biologie, farmacie en voedseltechniek. Dit zijn drie gebieden waar chemische selectiviteit en label-vrije detectie van essentieel belang zijn. Naast het CARS signaal worden er andere niet-lineaire signalen gelijktijdig gegenereerd. Deze signalen worden ook gedetecteerd om additionele informatie te verkrijgen. CARS, tweede-harmonische generatie microscopie en spontane Raman spectroscopie worden naast elkaar gebruikt om te komen tot een brede analyse van de processen. Er worden twee diepgaande studies gepresenteerd, namelijk het oplossingsgedrag van een geneesmiddel en de groei van botweefsel door cellen. 
Hoofdstuk zes bevat een selectie uit de metingen die gedaan zijn voor dit onderzoek en die de mogelijkheden van CARS microscopie laten zien. Naast de fysieke en biologische informatie die uit de beelden wordt verkregen, leiden de vormen en toegewezen kleuren vaak tot interessante patronen die de fantasie van de lezer stimuleren. Een aantal van deze artistieke beelden is verzameld om de lezer verder te leiden in de wondere wereld van microschaal kunst. 


\section{Dankwoord}

Voor jullie ligt het proefschrift waarin de resultaten staan die ik in de afgelopen vier jaar heb bereikt. Echter, onderzoek doe je niet alleen. Zonder de hulp van collega's, vrienden en familie op zowel wetenschappelijk, sociaal en ontspannend gebied was dit nooit gelukt. Daarom wil ik via deze weg een aantal van deze mensen specifiek bedanken.

Allereerst mijn dagelijks begeleider Herman. Ik weet nog goed hoe je me uiteindelijk hebt weten over te halen om te gaan promoveren. Jouw creatieve ideen, de zeer goede werksfeer in de groep, de excellente technische ondersteuning en de beloftes van een nieuwe microscoop, tafel en budget, besloten mij om dit nieuwe avontuur van vier jaar aan te gaan. En naar mijn mening waren dit vier zeer geslaagde jaren. We hebben de vakgroep op de kaart gezet binnen de CARS gemeenschap. Herman, ik wil je hartelijk danken voor alle begeleiding, de vele vijf minuutjes op je kamer en de mogelijkheden die je me hebt geboden om het onderzoek wereldwijd op conferenties te vertellen. Tevens heb ik er alle vertrouwen in dat die single oscillator zich ooit zal prijs geven. Al vraag ik me af wat je er vervolgens mee gaat doen.

En dan natuurlijk mijn promotor Jennifer. Toen jij de lege plek van het professorschap enkele maanden na mijn aanstelling weer vulde, drukte jij een nieuwe stempel op de groep. De naam van de vakgroep veranderde van Optische Technieken naar Optical Sciences, maar de belangrijkste verandering waren voor mij de groepsuitjes. Buiten dat deze uitjes erg gezellig waren, werden we meer dan alleen collega's in de groep. Ondanks de uitstekende begeleiding binnen de groep, miste ik zo nu en dan de directe wetenschappelijke interactie met jou als professor. Ik had 
graag meer jouw visie willen weten om de resultaten nog verder aan te kunnen scherpen.

Cees, in het tweede gedeelte van mijn promotie begon je een steeds belangrijkere rol te vervullen. Jij was namelijk mijn toegang tot de biomedische toepassingen. Op deze manier kon ik eindelijk de CARS microscopie toepassen op relevantere samples dan de gebruikelijke plastic bolletjes. Daarnaast ben ik na al die jaren nog steeds onder de indruk van de enorme kennis die je hebt van Raman spectra, al leken voor mij sommige vibraties die jij kwalificeerde als een enorme piek meer op ruis dan signaal.

Naast de begeleiders zijn de twee technici, Jeroen en Frans, van ongekende waarde geweest voor mijn onderzoek. Jeroen, jouw eeuwige geduld om mij te vertellen hoe een versterker werkt en hoe het ruisniveau en de quantum efficiency moeten worden berekend, waardeer ik enorm. Jij kreeg het altijd voor elkaar om mijn fysische ideen te vertalen naar een elektronische 'zwarte doos'. Je moet alleen nooit meer aan een promovendus vertellen dat er nog verborgen instelmogelijkheden zijn. Dit resulteerde in mijn geval altijd tot gaten in de mooie zwarte doosjes. Verder wil ik de secretaresse Karen nog bedanken. Buiten alle administratieve zaken die je voor me hebt geregeld, hebben we vele keren gezellig richting huis gefietst.

Besides the work I did, chapter 5 would never have been written if we had not had collaboration with other groups and people. Maike, it took us only two weeks to do the measurements, but the results are marvelous and the time we spent measuring was great fun. Vishnu, we had a great time in the lab. The boring and time-consuming data acquisition appeared to be faster with you around. Liesbeth, bedankt voor alle keren dat je de Raman setup voor mij hebt aangezet en hebt geholpen met het verkrijgen van de toch meestal saaie spectra.

Dan zijn er natuurlijk nog de mensen die de sfeer in "The Garage Bar \& Grill" (CARS-lab) bepaalden. Sytse, die mij de beginselen van de optica heeft bijgebracht, maar ook de haat jegens de Bohemian rhapsody van Queen heeft gegeven. Alexander, bedankt voor het proeflezen, de gedachten wisselingen en het code debuggen. Verder wil ik natuurlijk de afstudeer studenten, Philip, Kasper en Erik, die ik heb begeleid bedanken. Veel van jullie metingen hebben jullie misschien niet terug gevonden in dit proefschrift, maar het belangrijkste is wel dat jullie alle- 
maal een artikel hebben weten te publiceren met mijn naam erop. Erik, thanks for proof reading the thesis. Good luck with the continuation of the CARS project and keep focused.

Gelukkig kon ik naast het meestal donkere lab ook genieten van het ruime en bosrijke uitzicht van het 'Penthouse', waar het altijd gezellig en druk was. Robert, hoe lang heb ik wel niet moeten wachten op het beste plekje van de Hogekamp. Gelukkig heb ik hier nog enkele maanden van mogen genieten, voordat de vakgroep is verhuisd naar Carré. Oya, Di, Jincy, Ali and Dan, thanks for the pleasant time. Although we experienced the differences between cultures, we created a nice mixture within our group.

Mijn paranimfen, de gebroeders Hans en Jos Boschker, bedankt dat jullie mij zullen bijstaan tijdens de verdediging van mijn proefschrift. Maar natuurlijk vooral bedankt voor alle gezelligheid en de goede vriendschap.

Naast het werk was er tijd voor ontspanning bij de studenten klimvereniging TSAC. Ondanks dat ik al een oude rot ben geworden binnen de vereniging, is de gezelligheid tijdens trainingen, zaalavonden en weekendjes nog altijd hetzelfde. Regelmatig heb ik nieuwe inspiratie en ideeën gekregen tijdens een klimavond of weekend. Allen bedankt!

De laatste jaren van mijn onderzoek heb ik daarnaast veel ontspanning gevonden in het geocachen. Richard, bedankt voor het ontdekken en uitvoeren van deze nieuwe hobby tijdens een aantal vakanties. Alexander bedankt voor het geocachen tijdens de 'vrije' uurtjes op conferenties en op het werk en het verzinnen van nieuwe caches.

Het eind van de lijst is in zicht, maar niet voordat ik mijn ouders, Lex, schoonouders en andere naaste familieleden heb bedankt voor hun steun, vertrouwen en interesse in mijn werk. Ook al waren de plaatjes die ik maakte meestal interessanter dan de achterliggende fysica.

De laatste regels van dit proefschrift zijn voor jou, Rina. Jij gaf mij het excuus voor de negen tot vijf mentaliteit, welke niet geheel gebruikelijk is binnen de academische wereld. Je gaf mij echter wel de vrijheid om te kunnen werken wanneer dit nodig was. Bedankt voor de onvoorwaardelijke liefde, steun en het vertrouwen in mij. Hoewel de toekomst niet bekend is, we zien zullen wat deze voor ons in petto heeft.

\section{Martin}




\section{List of publications}

\section{Journal Articles}

- M. Jurna, J.P. Korterik, H.L. Offerhaus, and C. Otto, "Noncritical phasematched lithium triborate optical parametric oscillator for high resolution coherent anti-Stokes Raman scattering spectroscopy and microscopy", Appl. Phys. Lett., 89, 251116 (2006).

- M. Jurna, J.P. Korterik, C. Otto, and H.L. Offerhaus, "Shot noise limited heterodyne detection of CARS signals", Opt. Express, 15(23), 1520715213 (2007).

- M. Jurna, J.P. Korterik, C. Otto, J.L. Herek, and H.L. Offerhaus, "Background free CARS imaging by phase sensitive heterodyne CARS", Opt. Express, 16(20), 15863-15869 (2008).

- M. Jurna, M. Windbergs, C.J. Strachan, L. Hartsuiker, C. Otto, P. Kleinebudde, J.L. Herek, and H.L. Offerhaus, "Coherent Anti-Stokes Raman Scattering microscopy to monitor drug dissolution in different oral pharmaceutical tablets", Journal of Innovative Optical Health Sciences, 2(1), 37-43 (2009).

- M. Windbergs, M. Jurna, H.L. Offerhaus, J.L. Herek, P. Kleinebudde, and C.J. Strachan, "Chemical Imaging of Oral Solid Dosage Forms and Changes upon Dissolution Using Coherent Anti-Stokes Raman Scattering Microscopy", Anal. Chem., 81(6), 2085-2091 (2009).

- M. Jurna, J.P. Korterik, C. Otto, J.L. Herek, and H.L. Offerhaus, "Vibrational Phase Contrast Microscopy by Use of Coherent Anti-Stokes Raman Scattering", Phys. Rev. Lett., 103(4), 043905 (2009).

- P.F. Chimento, M. Jurna, H.S.P. Bouwmans, E.T. Garbacik, L. Hartsuiker, C. Otto, J.L. Herek, and H.L. Offerhaus, "High-resolution narrowband CARS spectroscopy in the spectral fingerprint region", J. Raman Spectrosc., 40(9), 1229-1233 (2009). 
- K. Orsel, E.T. Garbacik, M. Jurna, J.P. Korterik, C. Otto, J.L. Herek, and H.L. Offerhaus, "Heterodyne Interferometric Polarization Coherent Anti-Stokes Raman Scattering (HIP-CARS) Spectroscopy", J. Raman Spectrosc., accepted (2010).

\section{Journal Articles (in preparation)}

- M. Jurna, E.T. Garbacik, J.P. Korterik, C. Otto, J.L. Herek, and H.L. Offerhaus, "Visualizing resonances in the complex plane with vibrational phase contrast CARS", submitted

- M. Jurna, J.P. Korterik, C. Otto, J.L. Herek, and H.L. Offerhaus, "Third order phase-preserving chain for vibrational phase contrast CARS microscopy".

- V.V. Pully, M. Jurna, L.N. Azar, A. Lenferink, J.P. Korterik, V. Subramaniam, C.A. van Blitterswijk, J.L. Herek, H.L. Offerhaus and C. Otto, "How cells make bone".

\section{Proceeding Articles}

- M. Jurna, E. Büttner, J.P. Korterik, C. Otto, I. Rimke, and H.L. Offerhaus, "Shot noise limited heterodyne detection of CARS signals", Proceedings of SPIE, Volume 6860 Multiphoton Microscopy in the Biomedical Sciences VIII, 68600R (2008).

- M. Jurna, J.P. Korterik, C. Otto, J.L. Herek, and H.L. Offerhaus, "Background free CARS imaging by local phase detection", Proceedings of SPIE, Volume 7183 Multiphoton Microscopy in the Biomedical Sciences IX, 718310 (2009).

- M. Jurna, E.T. Garbacik, J.P. Korterik, C. Otto, J.L. Herek, and H.L. Offerhaus, "Vibrational phase contrast CARS microscopy for quantitative analysis", Proceedings of SPIE, Volume 7569 Multiphoton Microscopy in the Biomedical Sciences X, 75690F (2010).

\section{Patents}

- I. Rimke, E. Büttner, H.L. Offerhaus, C. Otto, and M. Jurna, "Verfahren und optische Anordnung zum Erzeugen eines nicht-linearen optischen Signals an einem durch ein Anregungsfeld angeregten Material sowie Verwendung des Verfahrens und der optischen Anordnung", Patent no. DE102007021378A1, 06.11.2008./WO2008135257.

\section{Dutch Articles}

- M. Jurna, "Vetkieren", Nederlands Tijdschrift voor Natuurkunde, 2(53) (2008). 
- H.L. Offerhaus en M. Jurna, "Vibrationele fasecontrastmicroscopie", Nederlands Tijdschrift voor Natuurkunde, 12,416-420 (2009).

\section{Conference contributions (oral)}

- M. Jurna, E. Büttner, J.P. Korterik, C. Otto, I. Rimke, and H.L. Offerhaus, "Shot noise limited heterodyne detection of CARS signals", SPIEBIOS Photonics West, San Jose CA, USA (2008).

- M. Jurna, J.P. Korterik, C. Otto, and H.L. Offerhaus, "Shot noise limited heterodyne detection of CARS signals", Nonlinear Microscopy and Optical Control (NMOC), Münster, Germany (2008).

- M. Jurna, J.P. Korterik, C. Otto, J.L. Herek, and H.L. Offerhaus, "Background free CARS imaging with local phase detection", NNV AMO, Lunteren, Netherlands (2008).

- M. Jurna, J.P. Korterik, C. Otto, J.L. Herek, and H.L. Offerhaus, "Background free CARS imaging with local phase detection", Physics@FOM, Veldhoven, Netherlands (2009).

- M. Jurna, J.P. Korterik, C. Otto , J.L. Herek, and H.L. Offerhaus, "Background free CARS imaging by local phase detection", SPIE-BIOS Photonics West, San Jose CA, USA (2009).

- M. Jurna, "CARS microscopy", Diner Pensant (invited), Den Haag, Netherlands (2009).

- M. Jurna, J.P. Korterik, C. Otto , J.L. Herek, and H.L. Offerhaus, "The New Generation CARS Microscopy", MicroNano Conference, Delft, Netherlands (2009).

- M. Jurna, E.T. Garbacik, J.P. Korterik, C. Otto , J.L. Herek, and H.L. Offerhaus, "Vibrational phase contrast CARS microscopy for quantitative analysis", SPIE-BIOS Photonics West, San Jose CA, USA (2010).

- M. Jurna V.V. Pully, J.P. Korterik, J.L. Herek, H.L. Offerhaus and C. Otto, "Investigation of early bone mineralization by CARS microscopy", Focus on microscopy, Shanghai, China (2010).

\section{Colloquium contributions (oral)}

- M. Jurna, "CARS microscopy - based on a green pumped OPO", Inorganic Materials Science colloquium, Enschede, Netherlands (2007).

- M. Jurna, J.P. Korterik, C. Otto, and H.L. Offerhaus, "CARS microscopy", MESA+ colloquium, Enschede, Netherlands (2007).

- M. Jurna, J.P. Korterik, C. Otto, and H.L. Offerhaus, "CARS microscopy - A special optical system for microscopy with chemical selectivity and high sensitivity", ICFO colloquium, Barcelona, Spain (2008). 
- M. Jurna, J.P. Korterik, C. Otto, J.L. Herek, and H.L. Offerhaus, "Phase coherent nonlinear spectroscopy and imaging", University of Lund colloquium, Lund, Sweden (2009).

\section{Media coverage (highlights)}

- M. Jurna, and H.L. Offerhaus, "Werking van medicijn op film", TV, News, RTV Oost, Hengelo (09 March 2009).

- M. Jurna, and H.L. Offerhaus, "Werking pillen op beeld vastgelegd", Internet, News, www.nu.nl (11 March 2009).

- M. Jurna, and H.L. Offerhaus, "Astma medicijn", Newspaper, Intermediair (19 March 2009).

- M. Jurna, and H.L. Offerhaus, "Film van afgifte pil", Scientific newspaper, Technisch weekblad (21 March 200)9

- M. Jurna, "Jonge wetenschappers in 'De wereld draait door"'. TV, De wereld draaid door, VARA, Amsterdam (13 April 2009).

- M. Jurna, "CARS microscoop", TV, Noorderlicht, VPRO, Hilversum (09 July 2009).

- M. Jurna, and H.L. Offerhaus, "De microscoop die bijna alles ziet", Newspaper, Twentsche courant Tubantia (18 April 2009).

- M. Jurna, "BioScapes Photo Contest: 15 Honorable Mentions, Scientific American staff selections from the contest runners-up", Scientific American, Internet (18 November 2009).

\section{Prizes and awards}

- M. Jurna, "Mesa+ photo contest 2009", 3rd prize (2009).

- M. Jurna, "Olympus BioScapes Digital Imaging Competition", Honorable Mention (2009). 
